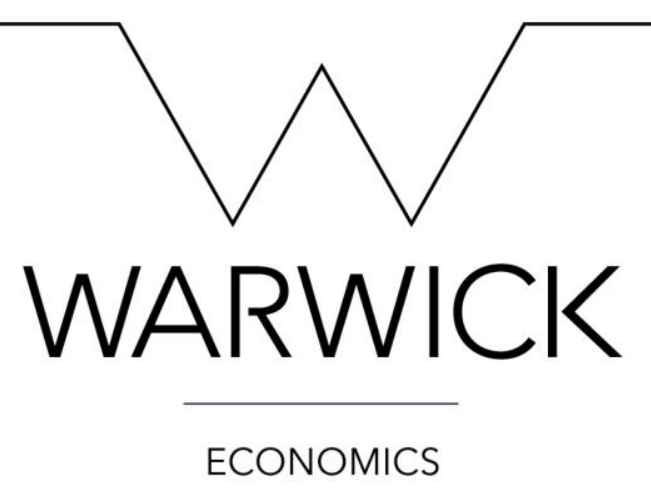

\title{
CRETA
}

Centre for Research in Economic Theory and its Applications

Discussion Paper Series

\section{Contests with Ex-Ante Target Setting}

Matthew J. Robertson

\section{CRETA}

Centre for Research in Economic Theory and its Applications

Department of Economics

University of Warwick, Coventry,

CV4 7AL, United Kingdom

warwick.ac.uk/fac/soc/economics/research/centres/creta/papers 


\title{
Contests with Ex-Ante Target Setting
}

\author{
Matthew J. Robertson*†
}

December 4, 2018

\begin{abstract}
I study contests in which each player is ranked by a scoring rule based on both her performance and how close this performance is to a private target, set before the contest. Each player's decision problem is to choose her target when performance is subject to a random component. I analyse the incentive properties of target setting, derive conditions on the primitives such that equilibria exist and characterise the players' behaviour. I show that target setting generates outcome uncertainty under a large class of conditions. In particular, neither private abilities nor perfectly correlated states are necessary. Target setting, therefore, has important implications in contest design as outcome uncertainty is a salient determinant of consumers' demand for contests.
\end{abstract}

Key Words: contests; target setting; competitive balance; incentives; incomplete information.

JEL Codes: C70; D81; D82; Z20.

\section{Introduction}

Competitive balance has long been thought to be important in professional sports (Rottenberg, 1956; El-Hodiri and Quirk, 1971). A sufficient degree of rivalry and competition between participants in a contest introduces ex-ante uncertainty about the outcome. This outcome uncertainty has been shown to be a salient determinant of the consumer demand for contests and is, consequently, of crucial importance for contest designers (Neale, 1964; Knowles et al., 1992; Forrest and Simmons, 2002; Szymanski, 2003b,a; Borland and MacDonald, 2003). Therefore, mechanisms that support outcome uncertainty and generate a greater degree of competitive balance are important for economic theory.

*Thanks are due to Alex Dickson, Marco Fongoni and Jasmin Droege for feedback on previous drafts and Ed Hopkins, Larry Samuelson and David Comerford for comments and suggestions that improved the paper. I would also like to thank participants of both the Scottish Graduate Programme in Economic's 2018 Residential Conference and the $71^{\text {st }}$ European Meeting of the Econometric Society for useful comments. Particular thanks are due to participants of the $14^{t h}$ European Meeting on Game Theory (SING14) for excellent comments, suggestions and discussion.

${ }^{\dagger}$ Coventry University, Department of Economics, William Morris Building, Gosford Street, Coventry, CV1 5DL.Email: ac9969@coventry.ac.uk. 
In this paper, I propose a mechanism that increases competitive balance in contests by creating outcome uncertainty. In my model this mechanism involves each player reporting a private performance target before the contest takes place. Contestants then take part in the contest where performance is determined by a combination of ability and the realisation of the state of nature. The random component means that each player is unable to perfectly estimate her performance when setting her target. Players are then ranked by a scoring rule that, amongst other properties, penalises negative deviations from target. The player with the highest score wins the prize. This rule-set rewards, and provides incentives for, a high performance as well as an accurate prediction of this performance. Each player's decision problem is to strategically choose a target setting rule that incorporates her private information about her own ability and her beliefs about both the abilities of her competitors and the possible states of nature.

To gain some intuition for why target setting can create outcome uncertainty, consider a contest between two players, whom I will refer to as Michael and Jane. Suppose that Jane is more able than Michael, and that this is common knowledge. Suppose further that there is a good and bad state of nature. In the bad state, players' performances are equal to their abilities, whilst in the good state, performances are greater than abilities. Without target setting Jane will win the contest, as her performance is always greater than Michael's. Upon introducing target setting, however, I show that Michael can obtain a strictly positive probability of winning. If the probability of the good state is sixty percent, Jane incorporates the positive effect of this state on her performance in her target sixty percent of the time. Michael, however, only does so forty percent of the time. Effectively, Michael gambles on the less likely state in the hope that Jane 'busts' by failing to achieve her target if the bad state is realised. With these strategies, Michael obtains a probability of winning of twenty-four percent. Therefore, by introducing target setting, there is now uncertainty about the outcome as Jane only wins the contest seventy-six percent of the time!

I generalise the above intuition by developing a model to study the effect that target setting has on players' incentives and their resulting equilibrium behaviour under a number of different conditions. I show that, generically, two salient properties of the contest emerge as players become almost sure of the state. First, the contest is incentive compatible, in a manner commensurate with the classic Vickrey (1961) auction. Second, due to incentive compatibility, the contest generates no outcome uncertainty. As each player truthfully reveals her private information, the outcome is determined entirely by the distribution of the players' abilities, akin to tournament models such as Lazear and Rosen (1981). The implication is that, if players can infer the state of nature through expertise or repetition, target setting will not be sufficient to create competitive balance. I then demonstrate that, when the states are equally likely, there are no pure strategy equilibria. This is because, when uncertainty about the state is maximal, the contest becomes a discoordination game with cyclical best responses (Pangallo et al., 2017). Intuitively, the unique mixed strategy equilibrium involves each player approximating her expected performance. Then, by analysing ex-post payoffs, I show that this setting generates outcome uncertainty; that is, less able players have a positive probability of winning.

In my two main results I derive sufficient conditions on the distribution of the players' abil- 
ities and the common prior over states such that pure strategy equilibria exist. These results provide minimum confidence levels each player must have about a state before she takes this state into account when setting her target. The intuition is that, once this minimum threshold has been surpassed, information about competitors' abilities is less valuable than more precise information about the state. This implies that outcome uncertainty is decreasing in the precision of this information. Moreover, when the skill gap between the players increases, the minimum confidence level decreases to reflect the reduced expected penalty from overestimation. A graphical representation of these conditions illustrates that for three quarters of the parameter space these pure strategies will not constitute equilibria. Therefore, mixed strategies will arise under the majority of information structures, leading to a large degree of outcome uncertainty.

Finally, I return to my motivating example and characterise equilibria when the players' abilities are common knowledge. I demonstrate that the intuition of less able players gambling on the less likely state holds in general; consequently, obtaining a strictly positive probability of winning. I then extend this analysis to a setting where, rather than facing a common state, players can face different realisations of the uncertainty. In this setting of imperfect correlation, each competitor plays according to her marginals and effectively ignores the correlation. The intuition is, therefore, akin to the setting with perfectly correlated states.

My analysis provides a number of salient insights. First, when players have precise information about the state, target setting is an incentive compatible mechanism. Therefore, target setting could be utilised by contest designers, and other economic agents, who wish to obtain accurate performance forecasts. Second, target setting yields outcome uncertainty and generates competitive balance under a wide range of conditions. Contest designers could, consequently, introduce target setting to increase consumer demand via outcome uncertainty. Beyond contests, these insights could be applied to more general economic settings ${ }^{1}$.

My target setting mechanism is inspired by several real world contests. In Olympic figure skating, for example, each contestant submits her planned performance to judges before the contest. Points are subtracted if her executed performance differs from this plan. Comparatively, in Olympic weight lifting, each contestant submits an initial weight to lift and then lifts heavier weights in successive increments. Contestants face a trade-off between starting at a low initial weight and having to perform a greater number of lifts or starting at a high initial weight and possibly failing to lift it within the three allowed attempts ${ }^{2}$. The main inspiration, however, is the

\footnotetext{
${ }^{1}$ One application could be sales-forecasting, which has been described as managers' most disliked activity (Herbig et al., 1993). Inaccurate forecasting leads to excess demand or supply when goods must be purchased in advance of sales. Many firms employ the sales-force composite approach; however, a significant proportion find this inaccurate (Mentzer and Cox Jr., 1984). A salient reason for this inaccuracy are the perverse incentives this method provides. If salespeople receive bonuses for achieving self-set targets, under-reporting is a dominant strategy. Therefore, a mechanism that provides incentives for accuracy would improve performance (Cox Jr., 1989).

My model predicts that, if demand shocks are known to salespeople, target setting would provide such incentives. Each salesperson's target would be her expected sales adjusted for any demand shocks. There would be no incentive to under-report. Such a situation could arise when demand shocks are predictable; for example, demand for seasonal products like Christmas trees and pumpkins. When demand shocks cannot be inferred, my results predict that target setting would allow less able salespeople to win the bonus. This could increase moral and encourage higher performance. However, if there is a wide discrepancy between the abilities of the salespeople, target setting benefits the relatively higher skilled more.

${ }^{2}$ See here for a summary of scoring in figure skating and here for a summary of rules in weight lifting,
} 
Apnea World Free-Diving Championship. Without the use of breathing equipment, contestants attempt to dive to the greatest depth in the ocean before successfully returning to the surface. The rules of this contest are very similar to the formal model in this paper as divers set private performance targets before diving. Therefore, I will often use the example of the diving contest to provide intuition for various assumptions and conditions ${ }^{3}$.

Conceptually, the contest I analyse is similar to those considered by Dixit (1987) and Che and Gale (1997), who study the incentive effects of pre-committing to effort and constraints or caps on effort, respectively. More recently, Fu et al. (2013) studied the effect of pre-contest communication. My contribution is to show that pre-committing to a target performance can increase competitive balance. My result concerning the outcome of the contest without uncertainty is akin to the tournament result found in Lazear and Rosen (1981), Green and Stokey (1983) and Nalebuff and Stiglitz (1983). My contribution is to demonstrate the conditions under which my model and the canonical tournament model are strategically equivalent. Moreover, this paper relates to the contemporary tournament literature that explores unconventional aspects such as the construction of optimal seeding (Groh et al., 2012), Round-Robin brackets (Arad and Rubinstein, 2013) and strategic bracket manipulation (Vong, 2017). My use of a scoring rule is analogous to the literature that studies the elicitation of an agent's belief about a probabilistic event (Hossain and Okui, 2013; Nelson and Bessler, 1989; Karni, 2009). My contribution is to demonstrate the conditions under which my scoring rule is proper in the sense of Savage (1971). Finally, I contribute to the literature on the economics of sport, such as Chiappori et al. (2002) and Walker and Wooders (2001). These authors analyse theoretical models and characterise mixed strategy Nash equilibria before taking the results to the data. Coloma (2012) extends Chiappori et al. (2002) to a setting with incomplete information. My contribution is to analyse a richer theoretical model with two forms of uncertainty and to characterise both pure and mixed strategies.

The paper is structured as follows. In Section 2, I lay out the general framework and establish existence of equilibrium. In Section 3, I make tractability assumptions to characterise the players incentives. In Section 4, I characterise equilibria in a number of distinct settings. In Section 5, I relax several of my tractability assumptions and consider extensions and generalisations. Finally, I conclude in Section 6 .

\section{General Framework}

I model the contest as a simultaneous move Bayesian game of incomplete information between a set $\mathscr{I}$ of players who compete for a fixed, indivisible prize that I normalise to unity ${ }^{4}$. Each player $i \in \mathscr{I}$ 's ability is $\theta_{i} \in \Theta \subset \mathbb{R}_{++}$and is private information. I assume that $\theta_{i}>\theta_{j}$ implies that player $i$ is more able than player $j$ for $i, j \in \mathscr{I}$. In the diving contest this assumption implies that, without taking the weather or water conditions into account, player $i$ can dive deeper than player

particularly sections 5.5.3-5.5.14

${ }^{3}$ Nestor (2014) provides an excellent discussion of the Apnea World Free-Diving Championship.

${ }^{4}$ This normalisation is without loss of generality and allows me to express each player's expected payoff as her probability of winning. 
$j$. Player $i$ 's private information is associated, in the sense of Harsanyi $(1967,1968 \mathrm{a}, \mathrm{b})$, with a belief about the abilities of her competitors $\left(\theta_{1}, \ldots, \theta_{i-1}, \theta_{i+1}, \ldots\right):=\theta_{-i} \in \Theta_{-i}=: \Theta^{\mid \mathscr{A}} \backslash\left\{\theta_{i}\right\}$ and a belief about the state of nature $k \in \mathscr{K} \subset \mathbb{R}_{+}$. The set $\mathscr{K}$ represents the uncertainty the players face. In the context of the diving contest, it could capture all possible weather and water conditions that exogenously affects the players' performances. Player $i$ 's beliefs are, therefore, an element of the set of joint probability distributions over the product set $\Theta_{-i} \times \mathscr{K}$ and are denoted $\rho\left(\theta_{-i}, k\right) \in \Delta\left(\Theta_{-i} \times \mathscr{K}\right)$. The set of states of the world contains all possible combinations of the realisation of the state and the players' abilities $\Omega=\mathscr{K} \times \prod_{i \in \mathscr{I}} \Theta$. Player $i$ 's performance is determined by the mapping $Q: \Theta \times \mathscr{K} \rightarrow \mathbb{R}_{++}$, which is a function of her ability and the realisation of the state. I assume this mapping is symmetric for all players; that is, $Q_{i}=Q_{j}=Q$ for each $i, j \in \mathscr{I}$. Moreover, for $\theta, \theta^{\prime}$ such that $\theta>\theta^{\prime}$ and $k, k^{\prime}$ such that $k>k^{\prime}$, I assume that $Q(\theta, \cdot)>Q\left(\theta^{\prime}, \cdot\right)$ and $Q(\cdot, k)>Q\left(\cdot, k^{\prime}\right)$, which together imply $Q(\theta, k)>Q\left(\theta^{\prime}, k^{\prime}\right)^{5}$.

Player $i$ 's action is to choose a performance target $t_{i} \in \mathscr{T} \subset \mathbb{R}_{++}{ }^{6}$. After each player has privately submitted her target, a referee collates the targets and makes them public as, for example, the linearly ordered set $(\mathbf{T}, \geq)=\left(t_{i}>t_{j}=t_{k}>\cdots\right)$. Player $i$ 's pure strategy is a mapping $\sigma_{i}: \Theta \rightarrow \mathscr{T}$, which is an injective function from her private information to her choice of target where $\sigma_{i}\left(\theta_{i}\right) \in \mathscr{T}$ for each $\theta_{i} \in \Theta$. Her mixed strategy is $\hat{\sigma}_{i}: \Theta \rightarrow \Delta(\mathscr{T})$, a probability distribution over pure strategies. A Bayesian-Nash equilibrium is a vector of strategies $\sigma^{*}=$ $\left(\sigma_{i}\left(\theta_{i}\right)\right)_{i \in \mathscr{I}}$ such that $\sigma_{i}^{*}\left(\theta_{i}\right)$ is a best response to $\left(\sigma_{1}^{*}\left(\theta_{1}\right), \ldots, \sigma_{i-1}^{*}\left(\theta_{i-1}\right), \sigma_{i+1}^{*}\left(\theta_{i+1}\right), \ldots\right):=$ $\sigma_{-i}^{*}\left(\theta_{-i}\right)$ for each $i \in \mathscr{I}$ and $\theta_{i} \in \Theta$.

Given the announced targets $(\mathbf{T}, \geq)$ and the vector of the players' performances when state $k$ has been realised $\mathbf{Q}_{k}:=\left(Q\left(\theta_{1}, k\right), \ldots, Q\left(\theta_{i}, k\right), \ldots\right)$, the players are ranked by a common knowledge scoring rule. This scoring rule possesses three salient properties.

Definition 1. The scoring rule is a function $S: Q(\Theta \times \mathscr{K}) \times \mathscr{T} \rightarrow \mathbb{R}_{++} . S$ has the following properties:

i Given $t_{i}$ such that $Q\left(\theta_{i}, k\right)<t_{i}, S\left(Q\left(\theta_{i}, k\right), t_{i}\right)<Q\left(\theta_{i}, k\right)$;

ii Given $t_{i}$ such that $Q\left(\theta_{i}, k\right) \geq t_{i}, S\left(Q\left(\theta_{i}, k\right), t_{i}\right)=t_{i}$;

iii Given $Q\left(\theta_{i}, k\right)$ and $t_{i}$ such that $Q\left(\theta_{i}, k\right)<t_{i}, S\left(Q\left(\theta_{i}, k\right), t_{i}\right)$ is decreasing in the penalty applied to negative deviations $\delta>0$.

Properties i and iii ensure that player $i$ 's score is decreasing in the difference between her target and her performance. Intuitively, negative deviations from target are costly. Property ii states that the target acts as an upper bound on player $i$ 's score, implying that she accrues no benefit from a performance greater than her target. Moreover, property ii implies that, if player $i$ 's performance is equal to her target, her score is also equal to this value. Finally, property iii

\footnotetext{
${ }^{5}$ This modelling choice means that, once the uncertainty has been resolved, each player's performance is given. I model performance in this way to focus attention on the problem of target setting, which is the unique aspect of this paper. Note that shifting focus away from effort choices is not without precedent, as Vong (2017) considers a strategic setting with costless effort.

6 "The contest officially starts the night before a dive, when divers secretly submit the proposed depths of the next day's dive attempts to a panel of judges. It's basically a bid, and there's gamesmanship involved as each diver tries to guess what the other divers will do" (Nestor, 2014).
} 
requires that, whenever player $i$ overestimates her performance, her score is decreasing in the penalty applied to negative deviations.

In state $k$ player $i$ has an ex-post payoff of $u_{i}^{k}(\mathbf{T})=\mathbb{1}_{S^{*}}(\mathbf{S})$, where $S^{*}=\max \left\{S\left(Q\left(\theta_{1}, k\right), t_{1}\right), \ldots\right\}$ $:=\max \mathbf{S}$. Therefore, as the contest is winner-take-all, player $i$ 's payoff is equal to unity if $S\left(Q\left(\theta_{i}, k\right), t_{i}\right)=S^{*}$ and zero otherwise. If the set $S^{*}$ is not a singleton, I adopt a tie-breaker rule that assigns each player whose score is in $S^{*}$ a $\frac{1}{\left|S^{*}\right|}$ probability of winning the contest. The following proposition specifies assumptions on this environment sufficient for the existence of a Bayes-Nash equilibrium.

Proposition 1. Suppose that $\mathscr{I}, \mathscr{K}, \mathscr{T}$ and $\Theta$ are finite. Then, a (potentially degenerate) mixed strategy Bayes-Nash equilibrium exists in the contest with ex-ante target setting.

Proof. Detailed proofs of all results are in Appendix A.

Therefore, player $i$ 's expected payoff is

$$
\mathbb{E}_{\theta, k}\left[u_{i}\left(\sigma, \theta_{i}\right)\right]=\sum_{\left(\theta_{-i}, k\right) \in \Theta_{-i} \times \mathscr{K}} \operatorname{Pr}\left(S \left(Q\left(\theta_{i}, k\right), \sigma_{i}\left(\theta_{i}\right)>S\left(Q\left(\theta_{j}, k\right), \sigma_{j}\left(\theta_{j}\right) \forall j \neq i\right) \rho\left(\theta_{-i}, k\right)\right.\right.
$$

when $\Theta$ and $\mathscr{K}$ are finite.

\section{Simplified Model}

I now invoke several tractability assumptions to characterise the incentives of the players and the resulting equilibrium behaviour. Assume that there are two players $\mathscr{I}=\{1,2\}$, each of whom can be one of two types $\Theta=\left\{\theta_{L}, \theta_{H}\right\}$, where $\theta_{H}>\theta_{L}$. The probability of player $i$ being relatively more able is $\operatorname{Pr}\left(\theta_{i}=\theta_{H}\right):=\mu \in[0,1]$, which is identical and independent for each $i \in \mathscr{I}$. Moreover, suppose there are two states of nature $\mathscr{K}=\left\{k_{L}, k_{H}\right\}$, where $k_{H}>k_{L}$. Denote a generic element of $\mathscr{K}$ as $k_{\ell}$ for $\ell \in\{L, H\}$. The common prior over the states is given by $\operatorname{Pr}\left(k=k_{L}\right):=\lambda \in[0,1]$. I assume that each player has the action set $\mathscr{T}=\left\{t_{L}, t_{H}\right\}=\left\{Q\left(\theta_{i}, k_{L}\right), Q\left(\theta_{i}, k_{H}\right)\right\}$, with generic element $t_{\ell}$. Finally, I assume that the scoring rule takes the form

$$
S\left(Q\left(\theta_{i}, k_{\ell}\right), t_{\ell}\right):=t_{\ell}-\delta \cdot \max \left\{0, t_{\ell}-Q\left(\theta_{i}, k_{\ell}\right)\right\}
$$

where, if $S\left(Q\left(\theta_{i}, k_{\ell}\right), t_{\ell}\right)=S\left(Q\left(\theta_{j}, k_{\ell}\right), t_{\ell}\right)$, each player wins with equal probability.

My first result states a sufficient condition on the size of the penalty applied to negative deviations from target.

Lemma 1. A sufficient condition for the scoring rule given in (2) to satisfy property i of Definition 1 is that the penalty applied to negative deviations is strictly greater than one.

Therefore, if a player's realised performance is less than her target, her score must be discounted to less than her performance. Intuitively, if $\delta \leq 1$, falling short of one's target 
is not punished. To see this, let $t^{\prime}>t$ and $Q\left(\theta_{i}, k\right)<t$. Then, if $\delta \leq 1$, I would have $S\left(Q\left(\theta_{i}, k\right), t^{\prime}\right)=(1-\delta) t^{\prime}+\delta Q\left(\theta_{i}, k\right)>(1-\delta) t+\delta Q\left(\theta_{i}, k\right)=S\left(Q\left(\theta_{i}, k\right), t\right)$ and player $i$ would always choose $t_{i}=\max \mathscr{T}$.

Combined with Lemma 1, the following two assumptions govern the players' incentives. These conditions determine the settings under which a relatively low ability player is able to obtain a greater score than a high ability player through strategic target setting.

\section{Assumption 1.}

$$
Q\left(\theta_{L}, k_{H}\right)>Q\left(\theta_{H}, k_{L}\right)
$$

Intuitively, when the state is good, a less able player will perform better than a highly able player who competes in the bad state. Without this assumption, a high ability player would always win by setting her performance target equal to her ability; subsequently, removing all outcome uncertainty.

\section{Assumption 2.}

$$
Q\left(\theta_{H}, k_{H}\right)-Q\left(\theta_{H}, k_{L}\right)>\frac{Q\left(\theta_{H}, k_{H}\right)-Q\left(\theta_{L}, k_{L}\right)}{\delta} .
$$

The intuition is that, when a less able player perfectly estimates his performance, his score is greater than that of a high ability player who overestimates her performance ${ }^{7}$. This assumption increases the set of scenarios in which a less able player could obtain a greater score than a high ability player through strategic target setting. Assumption 2 is more likely to hold when the skill gap between high and low ability players is relatively small, which implies the variance in the players' abilities is not too large ${ }^{8}$.

\section{Equilibrium Analysis}

\subsection{Equilibrium without Aggregate Uncertainty}

I first study Bayes-Nash equilibria when it is common knowledge that $\mathscr{K}=\{k\}$. If the state is known then $\operatorname{Pr}\left(k=k_{L}\right) \in\{0,1\}$. The purpose of this analysis is to elucidate the incentive properties of the target setting mechanism when there is no aggregate uncertainty. The ex-post payoffs when the state is neutral under Assumption 1 and Assumption 2 hold are illustrated in the strategic form game of Figure 3 in Appendix A. Each payoff pair is constant sum to one. As the players' abilities are identically and independently distributed, the probabilities associated with the strategic form games are

$$
\begin{gathered}
\operatorname{Pr}\left(\theta_{1}=\theta_{H} \cap \theta_{2}=\theta_{H}\right)=\operatorname{Pr}\left(\theta_{i}=\theta_{H}\right) \operatorname{Pr}\left(\theta_{i}=\theta_{H}\right), \\
\operatorname{Pr}\left(\theta_{1}=\theta_{H} \cap \theta_{2}=\theta_{L}\right)=\operatorname{Pr}\left(\theta_{1}=\theta_{L} \cap \theta_{2}=\theta_{H}\right)=\operatorname{Pr}\left(\theta_{i}=\theta_{H}\right) \operatorname{Pr}\left(\theta_{i}=\theta_{L}\right),
\end{gathered}
$$

\footnotetext{
${ }^{7}$ When contrasting high and low ability players I will refer to them as 'her' and 'him', respectively, as in the introduction.

${ }^{8}$ To see this, assume $Q(\theta, k)=\theta+k$ and $k_{L}:=0$. Then, Assumption 2 is equivalent to $k_{H}(\delta-1)>\theta_{H}-\theta_{L}$, and, for fixed $\delta$ and $k_{H}$, is more likely to hold when the difference $\theta_{H}-\theta_{L}$ is small.
} 
and

$$
\operatorname{Pr}\left(\theta_{1}=\theta_{L} \cap \theta_{2}=\theta_{L}\right)=\operatorname{Pr}\left(\theta_{i}=\theta_{L}\right) \operatorname{Pr}\left(\theta_{i}=\theta_{L}\right)
$$

Using these, I derive the players' expected payoffs to analyse the Bayesian strategic form of the game. For example, fix $\sigma_{j}\left(\theta_{j}\right)=\left(Q\left(\theta_{H}, k_{L}\right), Q\left(\theta_{L}, k_{L}\right)\right)$, which should be read as play $Q\left(\theta_{H}, k_{L}\right)$ when $\theta_{j}=\theta_{H}$ and play $Q\left(\theta_{L}, k_{L}\right)$ when $\theta_{j}=\theta_{L}$. Then, when $\sigma_{i}\left(\theta_{i}\right)=\left(Q\left(\theta_{H}, k_{L}\right), Q\left(\theta_{L}, k_{H}\right)\right)$, player $i$ 's expected payoff is

$$
\begin{aligned}
\mathbb{E}_{\theta}\left[u_{i}\left(\sigma, \theta_{i}\right)\right]=\mathbb{E}_{\theta}\left[u_{i}\left(\left(\sigma_{i}\left(\theta_{i}\right), \sigma_{j}\left(\theta_{j}\right)\right), \theta_{i}\right)\right] & =\underbrace{\mu^{2} \cdot \frac{1}{2}}_{\operatorname{Pr}\left(S_{i} \geq S_{j} \mid \theta_{i}=\theta_{j}=\theta_{H}\right)}+\underbrace{\mu(1-\mu) \cdot 1}_{\operatorname{Pr}\left(S_{i} \geq S_{j} \mid \theta_{i}=\theta_{H}, \theta_{j}=\theta_{L}\right)} \\
& +\underbrace{\mu(1-\mu) \cdot 0}_{\operatorname{Pr}\left(S_{i} \geq S_{j} \mid \theta_{i}=\theta_{L}, \theta_{j}=\theta_{H}\right)}+\underbrace{(1-\mu)^{2} \cdot 0}_{\operatorname{Pr}\left(S_{i} \geq S_{j} \mid \theta_{i}=\theta_{j}=\theta_{L}\right)}=\mu\left(1-\frac{\mu}{2}\right),
\end{aligned}
$$

where $S_{i}:=S\left(Q\left(\theta_{i}, k_{L}\right), \sigma_{i}\left(\theta_{i}\right)\right)$. Under these conditions I find that, without aggregate uncertainty, each player sets her performance target equal to her ability; therefore, truthfully revealing her private information.

Proposition 2. Suppose that either Assumption 1 and Assumption 2 hold or Assumption 1 holds but Assumption 2 does not. Then, if $\operatorname{Pr}\left(k=k_{\ell}\right)=1$ for $\ell \in\{L, H\}$, the strategy profile $\sigma_{i}^{*}\left(\theta_{i}\right)=Q\left(\theta_{i}, k_{\ell}\right)$ for $i \in \mathscr{I}$ is the unique pure strategy Bayes-Nash equilibrium for all $\operatorname{Pr}\left(\theta_{i}=\right.$ $\left.\theta_{H}\right)$.

The intuition is akin to the equilibrium of second price auctions, where each bidder optimally bids her true value. When the state is neutral ${ }^{9}$, for example, each player does not benefit from a strategy that over-or-underestimates her performance relative to her ability. In either case, her score would be less than if she had set her target equal to her ability. The target setting mechanism is, therefore, ex-post dominant strategy incentive compatible. Consequently, the player with the highest ability will win. I show in the following subsection that this finding generalises to a setting when the players are almost sure of the state ${ }^{10}$.

If players are able to accurately deduce the state, one conclusion is that the target setting mechanism may be superfluous. Such a situation could arise through expertise, repetition, or if the effect of the state was very small ${ }^{11}$. Therefore, a simple rank order tournament would induce the same outcome. A second implication is that there will not be outcome uncertainty. Contest designers may then wish to choose this mechanism when the state is known if their goal is incentive compatibility rather than outcome uncertainty.

\subsection{Equilibria with Aggregate Uncertainty}

I now revert to the assumption that $\mathscr{K}=\left\{k_{L}, k_{H}\right\}$ and derive the players' expected payoffs. To do so I take the expected payoffs in Figure 4 and Figure 6 and weight them using the common

\footnotetext{
${ }^{9}$ That is, when $k_{L}:=0$.

${ }^{10}$ I will show the result holds as $\operatorname{Pr}\left(k=k_{\ell}\right) \rightarrow 1$

${ }^{11}$ Let $Q\left(\theta_{i}, k_{H}\right)=\theta_{i}+k_{H}$ and $Q\left(\theta_{i}, k_{L}\right)=\theta_{i}$. Then, if the effect of the state is small, a performance target equal to ability remains a dominant strategy as $\lim _{k_{H} \searrow 0} Q\left(\theta_{i}, k_{H}\right)=Q\left(\theta_{i}, k_{L}\right)$.
} 
prior $\operatorname{Pr}\left(k=k_{L}\right):=\lambda$. The expectation is now taken over both the distribution of the players' abilities and the distribution over states. For example, player $i$ 's expected payoff is now

$$
\mathbb{E}_{\theta, k}\left[u_{i}\left(\left(\sigma_{i}\left(\theta_{i}\right), \sigma_{j}\left(\theta_{j}\right)\right), \theta_{i}\right)\right]=\underbrace{\lambda \cdot \mu\left(1-\frac{\mu}{2}\right)}_{\mathbb{E}_{\theta}\left[\operatorname{Pr}\left(S_{i} \geq S_{j} \mid k=k_{L}\right)\right]}+\underbrace{(1-\lambda) \cdot\left(1-\frac{\mu^{2}}{2}\right)}_{\mathbb{E}_{\theta}\left[\operatorname{Pr}\left(S_{i} \geq S_{j} \mid k=k_{H}\right)\right]},
$$

when $\sigma_{i}\left(\theta_{i}\right)=\left(Q\left(\theta_{H}, k_{L}\right), Q\left(\theta_{L}, k_{H}\right)\right)$ and $\sigma_{j}\left(\theta_{j}\right)=\left(Q\left(\theta_{H}, k_{L}\right), Q\left(\theta_{L}, k_{L}\right)\right)$. The players' expected payoffs are now defined entirely in terms of the primitives of the model due to the assumption that the prize is equal to unity. I first demonstrate that, when uncertainty about the state is maximal, the contest becomes a discoordination game with cyclical best responses.

Proposition 3. Suppose Assumption 1 and Assumption 2 hold. Then, if $\operatorname{Pr}\left(k=k_{L}\right)=\operatorname{Pr}(k=$ $\left.k_{H}\right)$, there are no pure strategy Bayes-Nash equilibria for all $\operatorname{Pr}\left(\theta_{i}=\theta_{H}\right)$.

Intuitively, given my assumption on the players' action set, each player is unable to incorporate the expected effect of the states in her target. Players are, therefore, left without mutually consistent best responses in pure strategies à la matching pennies. Instead, I show that it is optimal for each player to mix over her available strategies to approximate her expected performance.

Proposition 4. Suppose Assumption 1 and Assumption 2 hold. Then, if $\operatorname{Pr}\left(k=k_{L}\right)=\operatorname{Pr}(k=$ $\left.k_{H}\right)$, the strategy profile $\hat{\sigma}_{i}\left(\theta_{i}\right)=\mathbb{E}_{k}\left[Q\left(\theta_{i}, k\right)\right]$ for $i \in \mathscr{I}$ approximates the mixed-strategy BayesNash equilibrium for all $\operatorname{Pr}\left(\theta_{i}=\theta_{H}\right)$.

An important implication of this result is that the target setting mechanism will lead to outcome uncertainty. By looking at ex-post payoffs it is easy to see that a less able player will win one quarter of the time. Therefore, if uncertainty about the state is maximal, contest designers can successfully employ target setting to introduce a degree of competitive balance.

Unlike Proposition 2, when Assumption 2 does not hold the equilibrium behaviour of the players changes significantly. By increasing the gap between the players' abilities the possible penalty that results from incorrectly overestimating one's performance is reduced.

Corollary 1. Suppose Assumption 1 holds but Assumption 2 does not. Then, if $\operatorname{Pr}\left(k=k_{L}\right)=$ $\operatorname{Pr}\left(k=k_{H}\right)$, the strategy profile $\sigma_{i}^{*}\left(\theta_{i}\right)=Q\left(\theta_{i}, k_{H}\right)$ for $i \in \mathscr{I}$ is the unique pure strategy BayesNash equilibrium, by iterated elimination of weakly dominated strategies, for all $\operatorname{Pr}\left(\theta_{i}=\theta_{H}\right)$.

Intuitively, it becomes a dominant strategy for players to use a more optimistic target given the lower risk of overestimation. I use iterated elimination of weakly dominated strategies to remove equilibria in which the players only behave optimistically given a particular realisation of ability. By doing so, I pin down a unique equilibrium that is independent of the players' abilities. An implication of this result is that the target setting mechanism will be less effective at generating outcome uncertainty as the skill gap between players increases.

I now consider the general case when the common prior over states is unrestricted. My motivation is to derive sufficient conditions on this common prior and the distribution of the players' 
abilities such that pure strategy equilibria exist. These conditions provide a characterisation of the information structures that will not generate outcome uncertainty. I can then comment on the relative magnitude of information structures that will lead to outcome uncertainty.

Theorem 1. Suppose Assumption 1 and Assumption 2 hold. Then $\operatorname{Pr}\left(\theta_{i}=\theta_{H}\right) \in \tilde{\Pi}:=(2 \lambda, 1-$ $2 \lambda)$ implies that $\operatorname{Pr}\left(k=k_{L}\right) \in(0,1 / 4)$ and the strategy profile $\sigma_{i}^{*}\left(\theta_{i}\right)=Q\left(\theta_{i}, k_{H}\right)$ for $i \in \mathscr{I}$ is the unique pure strategy Bayes-Nash equilibrium.

Conversely, $\operatorname{Pr}\left(\theta_{i}=\theta_{H}\right) \in \hat{\Pi}:=(2(1-\lambda), 2 \lambda-1)$ implies that $\operatorname{Pr}\left(k=k_{L}\right) \in(3 / 4,1)$ and the strategy profile $\sigma_{i}^{*}\left(\theta_{i}\right)=Q\left(\theta_{i}, k_{L}\right)$ for $i \in \mathscr{I}$ is the unique pure strategy Bayes-Nash equilibrium.

The intuition is that there is a minimum level of confidence each player must have in a state for her to incorporate this state in her performance target. Specifically, one state must be at least three times more likely than the other. This is not the only necessary condition, however. At this minimum confidence level each player must be maximally uncertain about the abilities of her competitors. If she is strictly more likely to face a high ability competitor, for example, she will need to be even more confident in one state arising. These conditions are illustrated in Figure 1.

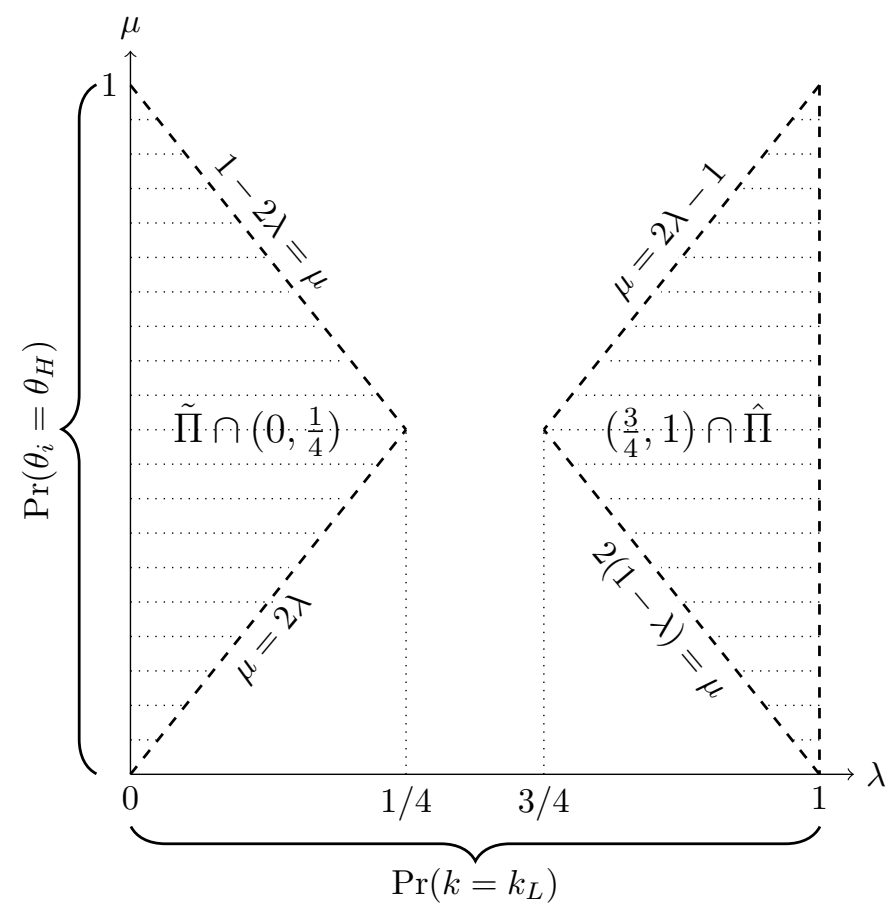

Figure 1: Information structures that lead to pure strategies.

There are several conclusions that can be drawn from this result. The first is that information about the state, condition upon being above the minimum confidence level, is more valuable than information about competitors' abilities. Intuitively, as the probability of a state approaches one, the distribution of the players' abilities becomes less of a concern. Therefore, in the limit, the players' behaviour approaches that detailed in Proposition 2. A second conclusion is that mixed 
strategies, or strategies that are conditional upon a particular realisation of ability, will arise for the majority of information structures. To see this note that

$$
\Pi:=\left[\tilde{\Pi} \cap\left(0, \frac{1}{4}\right)\right] \cup\left[\hat{\Pi} \cap\left(\frac{3}{4}, 1\right)\right] \subset\{(\mu, \lambda):(\mu, \lambda) \in[0,1] \times[0,1]\}
$$

spans one quarter of all possible information structures. Therefore, for three quarters of all information structures, the pure strategies detailed in Theorem 1 will not constitute equilibria. The implication is that the target setting mechanism will lead to outcome uncertainty under the majority of information structures. This is an important conclusion as it implies that, when the goal is to introduce competitive balance, target setting is sufficient in the vast majority of cases.

Similarly to Corollary 1, the players' behaviour changes significantly when Assumption 2 does not hold.

Theorem 2. Suppose Assumption 1 holds but Assumption 2 does not. Then, if $\operatorname{Pr}\left(k=k_{L}\right)<$ $\operatorname{Pr}\left(k=k_{H}\right)$, the strategy profile $\sigma_{i}^{*}\left(\theta_{i}\right)=Q\left(\theta_{i}, k_{H}\right)$ for $i \in \mathscr{I}$ is the unique pure strategy BayesNash equilibrium for all $\operatorname{Pr}\left(\theta_{i}=\theta_{H}\right)$.

Intuitively, as the potential penalty arising from overestimation is reduced, each player requires a lower degree of confidence in the performance enhancing state to take it into account in her target. In addition, once beliefs pass this minimum threshold, the players' equilibrium strategies become independent of the distribution of abilities. This is an extreme form of the intuition underlying Theorem 1, where the value of information about competitors' abilities is decreasing in information about the state. The set of information structures that yields pure strategies when Assumption 2 does not hold is illustrated in Figure 2. This change in information structures has one salient implication: pure strategies will now arise for the majority of information structures; specifically, for five eighths of the parameter space. Therefore, if the skill gap between high and low ability players is relatively large, the target setting mechanism will only generate outcome uncertainty under three eighths of information structures. The conclusion is that, whilst target setting continues to introduce outcome uncertainty, the degree of competitive balance created when the players' abilities are highly disparate is reduced.

\section{Extensions}

\subsection{Equilibria when Abilities are Common Knowledge}

I now return to the motivating example from the introduction in a formal setting and analyse equilibria when the players' abilities are common knowledge. The motivation for this extension is to develop an understanding of the effect of a published ranking on the players' incentives and behaviour ${ }^{12}$. Moreover, this analysis enables me to shed light on whether private information

\footnotetext{
${ }^{12}$ I have focused on the case when abilities are private for two reasons. The first is that a published ranking should have less impact under this mechanism as it is the size of the skill gap that is important. A published ranking that places player $i$ above player $j$ does not provide information about how much more able player $i$ is. The second reason is that private abilities allow me to study a more general setting that encompasses common knowledge abilities as a special case.
} 


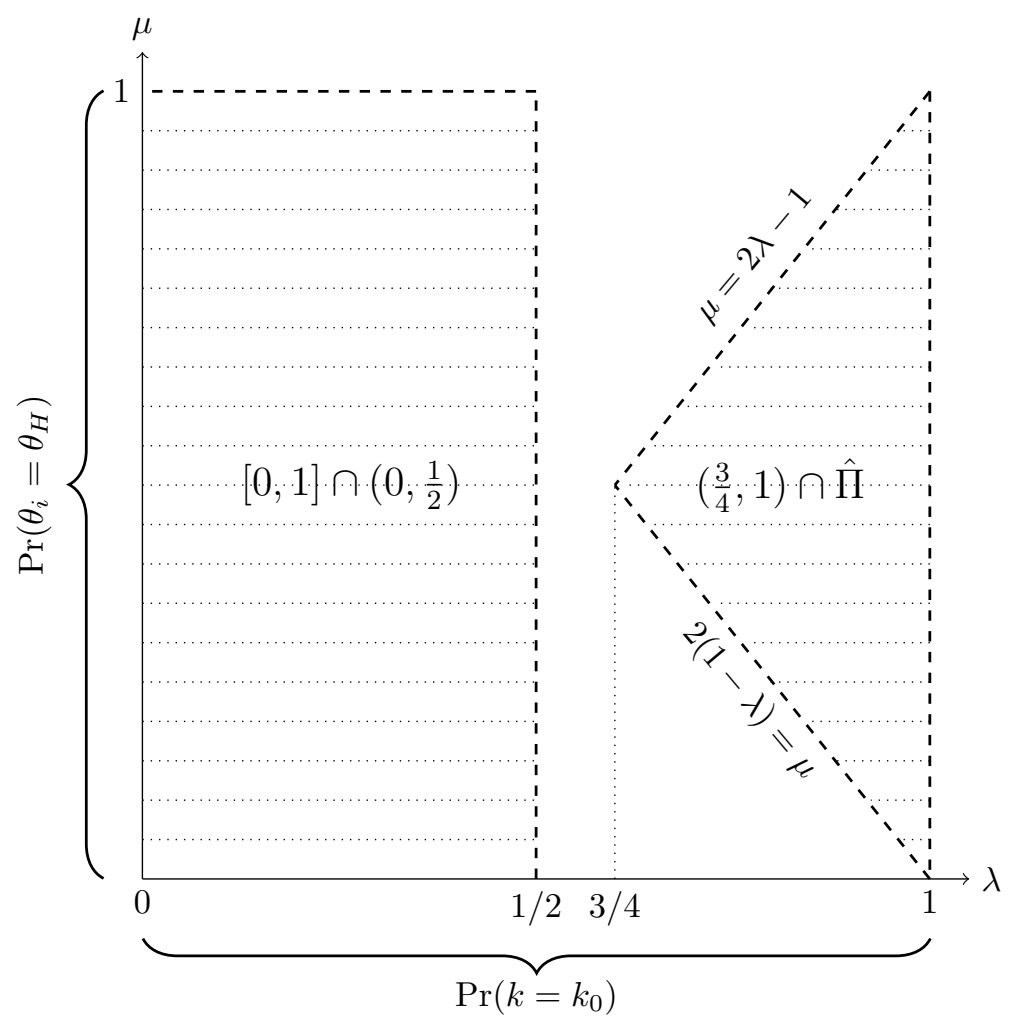

Figure 2: Information structures that lead to pure strategies when Assumption 2 does not hold.

about abilities is necessary for outcome uncertainty.

I first suppose that the two players are equal in ability. In this case, each player incorporates the effect of the state that is relatively more likely in her target setting.

Proposition 5. Suppose Assumption 1 and Assumption 2 hold and that $\theta_{1}=\theta_{2}$ is common knowledge. Then, if $\operatorname{Pr}\left(k=k_{L}\right)>\operatorname{Pr}\left(k=k_{H}\right)$, the strategy profile $\sigma_{i}^{*}\left(\theta_{i}\right)=Q\left(\theta_{i}, k_{L}\right)$ for $i \in \mathscr{I}$ is the unique pure strategy dominance solvable Nash equilibrium. Conversely, if $\operatorname{Pr}\left(k=k_{H}\right)>$ $\operatorname{Pr}\left(k=k_{L}\right)$, the strategy profile $\sigma_{i}^{*}\left(\theta_{i}\right)=Q\left(\theta_{i}, k_{H}\right)$ is the unique pure strategy dominance solvable Nash equilibrium.

When one state is relatively more likely than the other, each player's expected payoff is higher from setting a performance target that takes this state into account. Intuitively, this ensures that each player wins with equal probability.

I now consider the more interesting setting when it is common knowledge that one player is more able than the other. When the players'abilities are asymmetric there are no pure strategy equilibria as the game reverts to a discoordination game. Instead, in the mixed strategy equilibrium, the more able player acts relatively prudently. She places more probability mass on the strategy that is in line with the common prior; that is, if the good state is more likely she plays the strategy that incorporates that state relatively more often. Conversely, the less able player gambles on the less likely state. If the bad state is more likely, for example, he sets his 
performance target to take account of the good state relatively more often.

Proposition 6. Suppose Assumption 1 and Assumption 2 hold. Then, if $\theta_{1}=\theta_{H}>\theta_{L}=\theta_{2}$ is common knowledge, the strategies $\operatorname{Pr}\left(\sigma_{1}\left(\theta_{1}\right)=Q\left(\theta_{H}, k_{L}\right)\right)=\operatorname{Pr}\left(k=k_{L}\right)$ and $\operatorname{Pr}\left(\sigma_{2}\left(\theta_{2}\right)=\right.$ $\left.Q\left(\theta_{L}, k_{L}\right)\right)=\operatorname{Pr}\left(k=k_{H}\right)$ constitute a mixed-strategy Nash equilibrium.

The intuition is that, because the more able player has more to lose from overestimation, she effectively plays the odds and follows the common prior in her target setting. On the other hand, as the less able player has more to gain, he plays against the odds to maximise his probability of winning when the more able player guesses incorrectly. If the good state is more likely, the less able player sets his target in line with the bad state relatively more often, in the hope that the more able player fails to achieve her target when the bad state is realised ${ }^{13}$. Conversely, if the bad state is more likely, the less able player sets his target in line with the good state relatively more often. This implies that, given the more able player's strategy, the less able player's score will be greater when the good state is realised.

Proposition 6 highlights an important insight about the target setting mechanism: uncertainty about the players' abilities is not a necessary condition for outcome certainty. Therefore, even when there is a published ranking of the players' abilities, the target setting mechanism yields the less able player a strictly positive probability of winning; specifically, this probability is

$$
\operatorname{Pr}\left(k=k_{L}\right) \operatorname{Pr}\left(k=k_{H}\right)>0 .
$$

Conversely, without target setting the more able player would always win. The implication is that contest designers can implement this mechanism irrespective of whether players' abilities are private information.

To generalise the above result I will now assume that there is imperfect correlation between the realisation of the state the players face. The intuition for this assumption is that, in the diving context, the weather may change throughout the day as divers take part in the contest. Therefore, divers may compete under different weather conditions. Suppose that each player $i \in \mathscr{I}$ realises a state $k_{i}$ drawn from $\mathscr{K}_{i}=\left\{k_{L}, k_{H}\right\}:=\{0,1\}$ with joint probability distribution $\varrho\left(k_{1}, k_{2}\right) \in \Delta\left(\mathscr{K}_{1} \times \mathscr{K}_{2}\right)$. I assume this joint distribution satisfies

$$
\begin{gathered}
\operatorname{Pr}\left(k_{1}=k_{H} \cup k_{2}=k_{L}\right)=\operatorname{Pr}\left(k_{1}=k_{L} \cup k_{2}=k_{H}\right), \\
\operatorname{Pr}\left(k_{1}=k_{L} \cup k_{2}=k_{L}\right)>\operatorname{Pr}\left(k_{i}=k_{L} \cup k_{j}=k_{H}, i \neq j\right)>0
\end{gathered}
$$

and

$$
\sqrt{\operatorname{Pr}\left(k_{1}=k_{H} \cup k_{2}=k_{H}\right)}-\operatorname{Pr}\left(k_{1}=k_{H} \cup k_{2}=k_{H}\right)>\operatorname{Pr}\left(k_{i}=k_{L} \cup k_{j}=k_{H}, i \neq j\right),
$$

as this implies $1>\operatorname{corr}\left(k_{1}, k_{2}\right)>0$. Intuitively, these conditions capture the idea that it is more

\footnotetext{
13 "It's like playing poker", Trubridge told me." You are playing the other divers as much as you are playing yourself". The hope is that your foes will choose a shallower dive than you can do, or that they'll choose a deeper dive than they can do and end up "busting" (Nestor, 2014).
} 
likely that players face the same realisation of the state than experience different realisations. Yet, due to imperfect correlation, asymmetric realisations are no longer ruled out.

Proposition 7. Suppose Assumption 1 and Assumption 2 hold. Moreover, suppose that there is imperfect correlation between the states described by (3)-(5). Then, if $\theta_{1}=\theta_{H}>\theta_{L}=\theta_{2}$ is common knowledge, the strategies $\operatorname{Pr}\left(\sigma_{1}\left(\theta_{1}\right)=Q\left(\theta_{H}, k_{L}\right)\right)=\operatorname{Pr}\left(k_{1}=k_{L}\right)$ and $\operatorname{Pr}\left(\sigma_{2}\left(\theta_{2}\right)=\right.$ $\left.Q\left(\theta_{L}, k_{L}\right)\right)=\operatorname{Pr}\left(k_{2}=k_{H}\right)$ constitute a mixed-strategy Nash equilibrium.

When the states are imperfectly correlated each player effectively ignores the correlation and plays according to her marginal distribution. Specifically, the more able player acts in line with the common prior whilst the less able player gambles on the less likely state. The intuition and implications, therefore, carry over from the setting with perfect correlation between the states. The gain from this analysis is the insight that private abilities and perfectly correlated states are not necessary conditions for outcome uncertainty: the intuition generalises to more complex informational assumptions.

\subsection{Equilibria when Abilities are Continuously Distributed}

In this section I relax several of the tractability assumptions that have been employed hitherto. The main assumption throughout this section is that the players' abilities are continuously distributed. The purpose of this analysis is to elucidate the generality of Proposition 2 and to gain new insights from allowing, in certain respects, a richer environment. Throughout this section, however, I will assume that $Q(\theta, k)=\theta+k$ and that $k_{L}:=0$. These assumptions simplify the exposition yet I believe they are not necessary for the results.

\subsubsection{One State of Nature and a Continuous Action Set}

The first setting I consider is very general. I allow for any finite number of players and an arbitrary continuous distribution $\mathcal{F}$ over the players' abilities $[\underline{\theta}, \bar{\theta}]$. Moreover, I allow for the players' strategy set to be an arbitrary real interval with bounds determined by $\underline{\gamma}_{i}, \bar{\gamma}_{i}>0$, which need not be symmetric. The main restriction is the assumption of a single state. Player $i$ 's expected payoff in this setting is

$$
\mathbb{E}_{\theta}\left[u_{i}\left(\sigma, \theta_{i}\right)\right]=\int_{\Theta_{-i}} \operatorname{Pr}\left(S\left(Q\left(\theta_{i}, k_{L}\right), \sigma_{i}\left(\theta_{i}\right)>S\left(Q\left(\theta_{j}, k_{L}\right), \sigma_{j}\left(\theta_{j}\right)\right) \forall j \neq i\right) d \mathcal{F}\left(\theta_{-i}\right) .\right.
$$

I show that the intuition developed in Proposition 2 continues to hold under these conditions; that is, the target setting mechanism is ex-post dominant and incentive compatible when the state is known to competitors.

Proposition 8. Suppose $\mathscr{I}=\{1, \ldots, N\}, \theta_{i} \sim \mathcal{F}([\underline{\theta}, \bar{\theta}])$ for $i \in \mathscr{I}$ and $\mathscr{T}:=\left[\theta_{i}-\underline{\gamma}_{i}, \theta_{i}+\bar{\gamma}_{i}\right]$. Then, if $\operatorname{Pr}\left(k=k_{\ell}\right)=1$ for $\ell \in\{0,1\}$, the strategy profile $\sigma_{i}^{*}\left(\theta_{i}\right)=Q\left(\theta_{i}, k_{\ell}\right)$ for $i \in \mathscr{I}$ is the unique pure strategy Bayes-Nash equilibrium for all $\operatorname{Pr}\left(\theta_{i}=\theta_{H}\right)$.

The salient implication is that the target setting mechanism will incentivise truthful information revelation under a large class of conditions. Therefore, if the goal is incentive compatibility 
rather than competitive balance, this mechanism will perform well when information about the state is very precise.

The intuition behind the proof is that, when each player $j \in \mathscr{I} \backslash\{i\}$ truthfully reveals her private information, it is a dominant strategy for player $i$ to report her ability truthfully. Moreover, when each player $j \in \mathscr{I}$ does not truthfully report her information, player $i$ can increase her probability of winning by deviating to truthful revelation. In the former case, the sufficient conditions are

$$
\mathcal{F}\left(\theta_{i}\right)>\mathcal{F}\left(\theta_{i}-\underline{\gamma}_{i}\right) \text { and } \mathcal{F}\left(\theta_{i}\right)>\mathcal{F}\left(\theta_{i}-\bar{\gamma}_{i}(\delta-1)\right)
$$

Whilst, in the later case, the sufficient conditions are

$$
\begin{aligned}
& \mathcal{F}\left(\theta_{i}+\underline{\gamma}_{1}\right) \cdots \mathcal{F}\left(\theta_{i}+\underline{\gamma}_{i-1}\right) \mathcal{F}\left(\theta_{i}+\underline{\gamma}_{i+1}\right) \cdots \mathcal{F}\left(\theta_{i}+\underline{\gamma}_{N}\right) \\
&> \\
& \mathcal{F}\left(\theta_{i}-\underline{\gamma}_{i}+\underline{\gamma}_{1}\right) \cdots \mathcal{F}\left(\theta_{i}-\underline{\gamma}_{i}+\underline{\gamma}_{i-1}\right) \mathcal{F}\left(\theta_{i}-\underline{\gamma}_{i}+\underline{\gamma}_{i+1}\right) \cdots \mathcal{F}\left(\theta_{i}-\underline{\gamma}_{i}+\underline{\gamma}_{N}\right)
\end{aligned}
$$

and

$$
\begin{aligned}
& \mathcal{F}\left(\theta_{i}+\underline{\gamma}_{1}(\delta-1)\right) \cdots \mathcal{F}\left(\theta_{i}+\underline{\gamma}_{i-1}(\delta-1)\right) \mathcal{F}\left(\theta_{i}+\underline{\gamma}_{i+1}(\delta-1)\right) \cdots \mathcal{F}\left(\theta_{i}+\underline{\gamma}_{N}(\delta-1)\right)> \\
& \mathcal{F}\left(\theta_{i}-\left(\underline{\gamma}_{i}-\underline{\gamma}_{1}\right)(\delta-1)\right) \cdots \mathcal{F}\left(\theta_{i}-\left(\underline{\gamma}_{i}-\underline{\gamma}_{i-1}\right)(\delta-1)\right) \times \\
& \quad \times \mathcal{F}\left(\theta_{i}-\left(\underline{\gamma}_{i}-\underline{\gamma}_{i+1}\right)(\delta-1)\right) \cdots \mathcal{F}\left(\theta_{i}-\left(\underline{\gamma}_{i}-\underline{\gamma}_{N}\right)(\delta-1)\right) .
\end{aligned}
$$

Conditions (6)-(8) hold as $\mathcal{F}$ is nondecreasing by definition, $\underline{\gamma}_{i}, \bar{\gamma}_{i}>0$ for all $i \in \mathscr{I}$ and $\delta>1$ by Lemma 1 .

\subsubsection{Two States of Nature and a Discrete Action Set}

I now introduce two states of nature to the setting with continuously distributed abilities and assume players have the discrete action set detailed in Section 3. As before I approach the problem of characterising equilibria by deriving the conditions under which each player has a dominant strategy.

If each player $j \in \mathscr{I} \backslash\{i\}$ sets the lower target then player $i$ 's best response is to use this target if

$$
\begin{aligned}
& \mathbb{E}_{\theta, k}\left[u_{i}\left(\sigma^{\prime}, \theta_{i}\right)\right]=\left(\mathcal{F}\left(\theta_{i}\right)\right)^{N-1}> \\
& \operatorname{Pr}\left(k=k_{L}\right)\left(\mathcal{F}\left(\theta_{i}-k_{H}(\delta-1)\right)\right)^{N-1}+\operatorname{Pr}\left(k=k_{H}\right)\left(\mathcal{F}\left(\theta_{i}+k_{H}\right)\right)^{N-1} \\
& =\mathbb{E}_{\theta, k}\left[u_{i}\left(\left(\sigma_{i}^{\prime \prime}\left(\theta_{i}\right), \sigma^{\prime} \backslash\left\{\sigma_{i}\left(\theta_{i}\right)\right\}\right), \theta_{i}\right)\right] .
\end{aligned}
$$

Conversely, if each player $j \in \mathscr{I} \backslash\{i\}$ sets the higher target, player $i$ has a profitable deviation 
to the lower target if

$$
\begin{aligned}
\mathbb{E}_{\theta, k}\left[u_{i}\left(\left(\sigma_{i}^{\prime}\left(\theta_{i}\right), \sigma^{\prime \prime} \backslash\left\{\sigma_{i}\left(\theta_{i}\right)\right\}\right), \theta_{i}\right)\right] & \\
=\operatorname{Pr}\left(k=k_{L}\right)\left(\mathcal{F}\left(\theta_{i}+k_{H}(\delta-1)\right)\right)^{N-1}+\operatorname{Pr}\left(k=k_{H}\right)\left(\mathcal{F}\left(\theta_{i}-k_{H}\right)\right)^{N-1} & >\left(\mathcal{F}\left(\theta_{i}\right)\right)^{N-1}=\mathbb{E}_{\theta, k}\left[u_{i}\left(\sigma^{\prime \prime}, \theta_{i}\right)\right]
\end{aligned}
$$

Proposition 9. Suppose $\mathscr{I}=\{1, \ldots, N\}, \theta_{i} \sim \mathcal{F}([\underline{\theta}, \bar{\theta}])$ for $i \in \mathscr{I}$ and $\mathscr{T}:=\left\{Q\left(\theta_{i}, k_{L}\right), Q\left(\theta_{i}, k_{H}\right)\right\}$. Then, if (9) and (10) hold, the strategy profile $\sigma_{i}^{*}\left(\theta_{i}\right)=Q\left(\theta_{i}, k_{L}\right)$ is the unique pure strategy Bayes-Nash equilibrium.

Therefore, if (9) and (10) hold, each player setting the low performance target will constitute a dominant strategy Bayes-Nash equilibrium. Conversely, if these conditions do not hold, the higher performance target will constitute a dominant strategy Bayes-Nash equilibrium. Intuitively, as the neutral state becomes relatively more likely, the risk associated with the higher performance target increases.

To render these conditions more tractable I assume that there are two players whose abilities are uniformly distributed over the unit interval. These assumptions imply that (9) and (10) can be written as

$$
\lambda \int_{0}^{\theta_{i}+k_{H}(\delta-1)} d \theta_{j}+(1-\lambda) \int_{0}^{\theta_{i}-k_{H}} d \theta_{j}>\theta_{i}>\lambda \int_{0}^{\theta_{i}-k_{H}(\delta-1)} d \theta_{j}+(1-\lambda) \int_{0}^{\theta_{i}+k_{H}} d \theta_{j} .
$$

Analysis of (11) yields the following equilibrium characterisation.

Example 1. Suppose $\mathscr{I}=\{1,2\}, \theta_{i} \sim \mathcal{U}([0,1])$ for $i \in \mathscr{I}$ and $\mathscr{T}:=\left\{Q\left(\theta_{i}, k_{L}\right), Q\left(\theta_{i}, k_{H}\right)\right\}$. Then, if $\frac{1}{\operatorname{Pr}\left(k=k_{L}\right)}<\delta$, the strategy profile $\sigma_{i}^{*}\left(\theta_{i}\right)=Q\left(\theta_{i}, k_{L}\right)$ is the unique pure strategy BayesNash equilibrium. Conversely, if $\frac{1}{\operatorname{Pr}\left(k=k_{L}\right)}>\delta$, the strategy profile $\sigma_{i}^{*}\left(\theta_{i}\right)=Q\left(\theta_{i}, k_{H}\right)$ is the unique pure strategy Bayes-Nash equilibrium.

Intuitively, for a fixed penalty applied to negative deviations, as the probability of the neutral state increases, the equilibrium of Proposition 9 becomes relatively more likely. As this state becomes more likely, the expected penalty arising from overestimation increases and the expected gain from the higher performance target decreases. Conversely, when this state becomes less likely, the expected gain outweighs the potential penalty.

The gain from this analysis is an insight into the role played by the penalty applied to negative deviations from target. If the probability of the neutral state is held fixed, the size of this penalty uniquely determines equilibrium. The greater the penalty, the less willing players are to risk overestimation.

\section{Conclusion}

Target setting has two salient implications in contests. First, if each player is confident in her knowledge of the external factors that will influence her performance, target setting is incentive compatible. Each player will truthfully reveal her private information. Target setting will, 
therefore, be effective if contest designers wish to promote accurate performance forecasting. Second, in the vast majority of cases, target setting will yield outcome uncertainty and generate competitive balance. This finding relies on neither private abilities nor perfectly correlated uncertainty. My model predicts that contest designers could, therefore, introduce target setting and obtain contests where less able players have a strictly positive probability of winning. Empirical evidence suggests that, as a result, the consumer demand for such contests could increase; in particular, when the contests are between individual competitors.

Future research could focus on the dual problem of each player choosing both her effort and her target. This may yield new findings and could enable the model to capture some more realistic features. However, effort choices are not necessary to gain insights into the problem of competitive balance. 


\section{Appendices}

\section{A Proofs}

Proof of Proposition 1. Standard arguments imply that the results of Nash (1951) can be applied as both the distribution of the players' abilities and the distribution of the states of nature are common knowledge (Fudenberg and Tirole, 1991).

Proof of Lemma 1. This has been shown to hold in general in the main text. To see that this condition is sufficient given a particular form of production function suppose that $Q(\theta, k)=\theta+k$ and $k_{L}:=0$. Suppose further that player $i \in \mathscr{I}$ is of low ability so that $\theta_{i}=\theta_{L}$. This is without loss of generality as an analogous result holds when $\theta_{i}=\theta_{H}$. Then, supposing that the realised state of nature is $k_{L}$, player $i$ 's score from using the action $t_{L}=Q\left(\theta_{L}, k_{L}\right)$ is $S\left(Q\left(\theta_{L}, k_{L}\right), t_{L}\right)=\theta_{L}$. If instead, player $i$ used the action $t_{H}=Q\left(\theta_{L}, k_{H}\right)$, her score would be $S\left(Q\left(\theta_{L}, k_{L}\right), t_{H}\right)=\theta_{L}+k_{H}-\delta\left(\theta_{L}+k_{H}-\theta_{L}\right)$. Comparing these two scores yields the inequality $\theta_{L}>\theta_{L}+k_{H}-\delta\left(\theta_{L}+k_{H}-\theta_{L}\right)$, which reduces to $\delta>1$.

Proof of Proposition 2. I first consider the case when Assumption 1 and Assumption 2 hold. Suppose initially that $\mathscr{K}=\left\{k_{L}\right\}$, which implies the initial strategic form games are those given in Figure 3. Then, by following the procedure laid out in Subsection 4.1 for deriving $\mathbb{E}_{\theta}\left[u_{i}\left(\sigma, \theta_{i}\right)\right]$

2

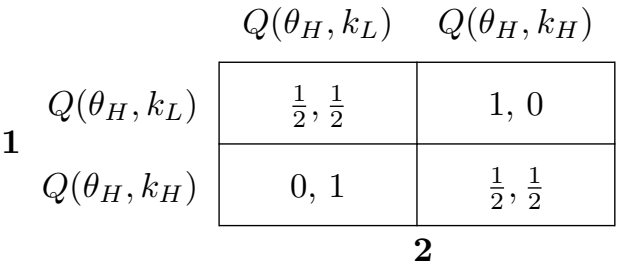

\begin{tabular}{|c|c|c|}
\hline & $Q\left(\theta_{H}, k_{L}\right)$ & $Q\left(\theta_{H}, k_{H}\right)$ \\
\hline$Q\left(\theta_{L}, k_{L}\right)$ & 0,1 & 1,0 \\
\hline$Q\left(\theta_{L}, k_{H}\right)$ & 0,1 & 0,1 \\
\hline
\end{tabular}

2

$Q\left(\theta_{L}, k_{L}\right) \quad Q\left(\theta_{L}, k_{H}\right)$

1

\begin{tabular}{c|c|c|}
\cline { 2 - 3 }$Q\left(\theta_{H}, k_{L}\right)$ & 1,0 & 1,0 \\
\cline { 2 - 3 }$Q\left(\theta_{H}, k_{H}\right)$ & 0,1 & 1,0 \\
\cline { 2 - 3 } & \multicolumn{2}{|c|}{$\mathbf{2}$}
\end{tabular}

1

\begin{tabular}{|c|c|c|}
$Q\left(\theta_{L}, k_{L}\right)$ & $\frac{1}{2}, \frac{1}{2}$ & 1,0 \\
\cline { 2 - 3 }$Q\left(\theta_{L}, k_{H}\right)$ & 0,1 & $\frac{1}{2}, \frac{1}{2}$ \\
\hline
\end{tabular}

Figure 3: Strategic Form Games when $\mathscr{K}=\left\{k_{L}\right\}$.

for each possible strategy profile $\sigma=\left(\sigma_{1}\left(\theta_{1}\right), \sigma_{2}\left(\theta_{2}\right)\right)$, I can produce the Bayesian strategic form of the game. This is illustrated in Figure 4. Note that, to save on notation, I will denote a strategy profile as, for example, $\sigma=\left(Q\left(\theta_{H}, k_{L}\right) Q\left(\theta_{L}, k_{H}\right), Q\left(\theta_{H}, k_{H}\right) Q\left(\theta_{L}, k_{L}\right)\right)$. This should be read player 1 sets a target of $Q\left(\theta_{H}, k_{L}\right)$ when $\theta_{1}=\theta_{H}$ and a target of $Q\left(\theta_{L}, k_{H}\right)$ when $\theta_{1}=\theta_{L}$ whilst player 2 sets a target of $Q\left(\theta_{H}, k_{H}\right)$ when $\theta_{2}=\theta_{H}$ and $Q\left(\theta_{L}, k_{L}\right)$ when $\theta_{2}=\theta_{L}$. 


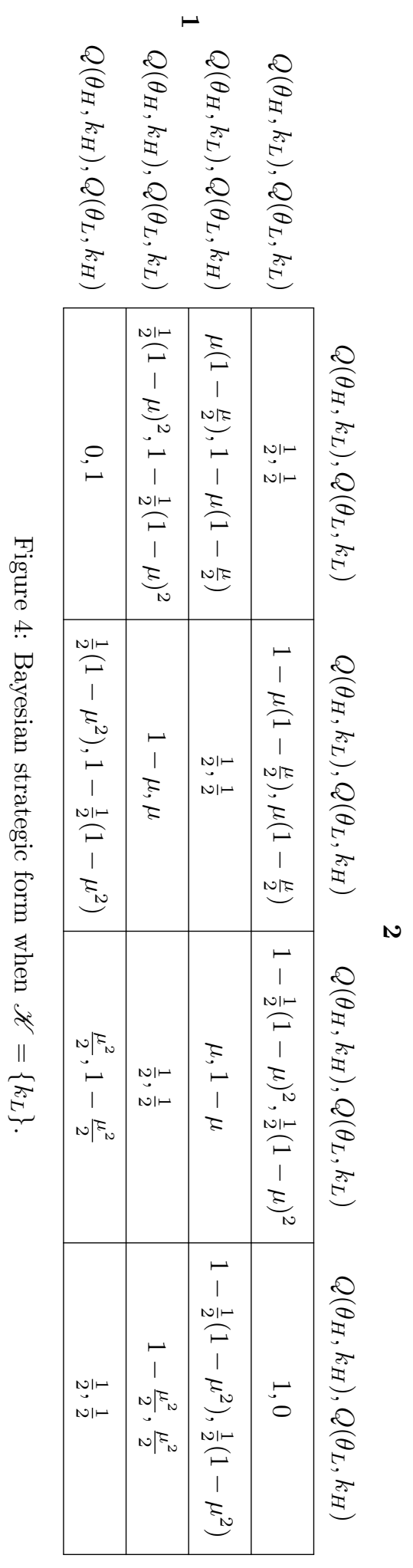


I will now show that, for each $i \in \mathscr{I}$, the strategy $\sigma_{i}\left(\theta_{i}\right)=\left(Q\left(\theta_{H}, k_{L}\right) Q\left(\theta_{L}, k_{L}\right)\right)$ is a best response to any strategy of player $j \in \mathscr{I} \backslash\{i\}$. Suppose player $j$ uses the strategy $\sigma_{j}\left(\theta_{j}\right)=$ $\left(Q\left(\theta_{H}, k_{L}\right) Q\left(\theta_{L}, k_{L}\right)\right)$, then, player $i$ 's strategy is a best response if

$$
\begin{aligned}
\mathbb{E}_{\theta}\left[u_{i}\left(\left(Q\left(\theta_{H}, k_{L}\right) Q\left(\theta_{L}, k_{L}\right), Q\left(\theta_{H}, k_{L}\right) Q\left(\theta_{L}, k_{L}\right)\right), \theta_{i}\right)\right] & \\
>\mathbb{E}_{\theta}\left[u_{i}\left(\left(Q\left(\theta_{H}, k_{L}\right) Q\left(\theta_{L}, k_{H}\right), Q\left(\theta_{H}, k_{L}\right) Q\left(\theta_{L}, k_{L}\right)\right), \theta_{i}\right)\right] & \Leftrightarrow \frac{1}{2}>\mu\left(1-\frac{\mu}{2}\right) \Leftrightarrow \frac{1}{2}(1-\mu)^{2}>0
\end{aligned}
$$

and

$$
\begin{aligned}
& \mathbb{E}_{\theta}\left[u_{i}\left(\left(Q\left(\theta_{H}, k_{L}\right) Q\left(\theta_{L}, k_{L}\right), Q\left(\theta_{H}, k_{L}\right) Q\left(\theta_{L}, k_{L}\right)\right), \theta_{i}\right)\right] \\
&>\mathbb{E}_{\theta}\left[u_{i}\left(\left(Q\left(\theta_{H}, k_{H}\right) Q\left(\theta_{L}, k_{L}\right), Q\left(\theta_{H}, k_{L}\right) Q\left(\theta_{L}, k_{L}\right)\right), \theta_{i}\right)\right] \\
& \Leftrightarrow \frac{1}{2}>\frac{1}{2}(1-\mu)^{2} \Leftrightarrow 1>\mu .
\end{aligned}
$$

Conditions (12) and (13) are satisfied by the assumption that $\operatorname{Pr}\left(\theta_{i}=\theta_{H}\right)=\mu \in(0,1)$ and, hence, player $i$ 's strategy is a best response to that of player $j$.

Suppose now that player $j$ uses the strategy $\sigma_{j}\left(\theta_{j}\right)=\left(Q\left(\theta_{H}, k_{L}\right) Q\left(\theta_{L}, k_{H}\right)\right)$. Player $i$ 's strategy is a best response to this if

$$
\begin{array}{r}
\mathbb{E}_{\theta}\left[u_{i}\left(\left(Q\left(\theta_{H}, k_{L}\right) Q\left(\theta_{L}, k_{L}\right), Q\left(\theta_{H}, k_{L}\right) Q\left(\theta_{L}, k_{H}\right)\right), \theta_{i}\right)\right] \\
>\mathbb{E}_{\theta}\left[u_{i}\left(\left(Q\left(\theta_{H}, k_{L}\right) Q\left(\theta_{L}, k_{H}\right), Q\left(\theta_{H}, k_{L}\right) Q\left(\theta_{L}, k_{H}\right)\right), \theta_{i}\right)\right] \\
\Leftrightarrow 1-\mu\left(1-\frac{\mu}{2}\right)>\frac{1}{2} \Leftrightarrow \frac{1}{2}>\mu\left(1-\frac{\mu}{2}\right),
\end{array}
$$

$$
\begin{aligned}
\mathbb{E}_{\theta}\left[u_{i}\left(\left(Q\left(\theta_{H}, k_{L}\right) Q\left(\theta_{L}, k_{L}\right), Q\left(\theta_{H}, k_{L}\right) Q\left(\theta_{L}, k_{H}\right)\right), \theta_{i}\right)\right] \\
>\mathbb{E}_{\theta}\left[u_{i}\left(\left(Q\left(\theta_{H}, k_{H}\right) Q\left(\theta_{L}, k_{L}\right), Q\left(\theta_{H}, k_{L}\right) Q\left(\theta_{L}, k_{H}\right)\right), \theta_{i}\right)\right] \\
\Leftrightarrow 1-\mu\left(1-\frac{\mu}{2}\right)>1-\mu \Leftrightarrow \frac{\mu^{2}}{2}>0
\end{aligned}
$$

and

$$
\begin{aligned}
& \mathbb{E}_{\theta}\left[u_{i}\left(\left(Q\left(\theta_{H}, k_{L}\right) Q\left(\theta_{L}, k_{L}\right), Q\left(\theta_{H}, k_{L}\right) Q\left(\theta_{L}, k_{H}\right)\right), \theta_{i}\right)\right] \\
& >\mathbb{E}_{\theta}\left[u_{i}\left(\left(Q\left(\theta_{H}, k_{H}\right) Q\left(\theta_{L}, k_{H}\right), Q\left(\theta_{H}, k_{L}\right) Q\left(\theta_{L}, k_{H}\right)\right), \theta_{i}\right)\right] \\
& \Leftrightarrow 1-\mu\left(1-\frac{\mu}{2}\right)>\frac{1}{2}\left(1-\mu^{2}\right) \Leftrightarrow \frac{1}{2}>0 .
\end{aligned}
$$

Condition (14) is equivalent to (12) and is, therefore, satisfied. Condition (15) is satisfied for $\mu \in(0,1)$ and (16) is trivially satisfied. Player $i$ 's strategy is thus a best response to player $j$ 's.

Next, suppose that player $j$ uses the strategy $\sigma_{j}\left(\theta_{j}\right)=\left(Q\left(\theta_{H}, k_{H}\right) Q\left(\theta_{L}, k_{L}\right)\right)$. Player $i$ 's 
strategy is a best response to this if

$$
\begin{gathered}
\mathbb{E}_{\theta}\left[u_{i}\left(\left(Q\left(\theta_{H}, k_{L}\right) Q\left(\theta_{L}, k_{L}\right), Q\left(\theta_{H}, k_{H}\right) Q\left(\theta_{L}, k_{L}\right)\right), \theta_{i}\right)\right] \\
>\mathbb{E}_{\theta}\left[u_{i}\left(\left(Q\left(\theta_{H}, k_{L}\right) Q\left(\theta_{L}, k_{H}\right), Q\left(\theta_{H}, k_{H}\right) Q\left(\theta_{L}, k_{L}\right)\right), \theta_{i}\right)\right] \\
\Leftrightarrow 1-\frac{1}{2}(1-\mu)^{2}>\mu \Leftrightarrow 1>\mu^{2},
\end{gathered}
$$

$$
\begin{aligned}
\mathbb{E}_{\theta}\left[u_{i}\left(\left(Q\left(\theta_{H}, k_{L}\right) Q\left(\theta_{L}, k_{L}\right), Q\left(\theta_{H}, k_{H}\right) Q\left(\theta_{L}, k_{L}\right)\right), \theta_{i}\right)\right] & \\
>\mathbb{E}_{\theta}\left[u_{i}\left(\left(Q\left(\theta_{H}, k_{H}\right) Q\left(\theta_{L}, k_{L}\right), Q\left(\theta_{H}, k_{H}\right) Q\left(\theta_{L}, k_{L}\right)\right), \theta_{i}\right)\right] & \Leftrightarrow 1-\frac{1}{2}(1-\mu)^{2}>\frac{1}{2} \Leftrightarrow 1>\mu
\end{aligned}
$$

and

$$
\begin{aligned}
& \mathbb{E}_{\theta}\left[u_{i}\left(\left(Q\left(\theta_{H}, k_{L}\right) Q\left(\theta_{L}, k_{L}\right), Q\left(\theta_{H}, k_{H}\right) Q\left(\theta_{L}, k_{L}\right)\right), \theta_{i}\right)\right] \\
&>\mathbb{E}_{\theta}\left[u_{i}\left(\left(Q\left(\theta_{H}, k_{H}\right) Q\left(\theta_{L}, k_{H}\right), Q\left(\theta_{H}, k_{H}\right) Q\left(\theta_{L}, k_{L}\right)\right), \theta_{i}\right)\right] \\
& \Leftrightarrow 1-\frac{1}{2}(1-\mu)^{2}>\frac{\mu^{2}}{2} \Leftrightarrow \frac{1}{2}+\mu(1-\mu)>0 .
\end{aligned}
$$

Conditions (17)-(19) are satisfied by the assumption of $\mu \in(0,1)$.

Finally, suppose that player $j$ uses the strategy $\sigma_{j}\left(\theta_{j}\right)=\left(Q\left(\theta_{H}, k_{H}\right) Q\left(\theta_{L}, k_{H}\right)\right)$. Player $i$ 's strategy is a best response to this if

$$
\begin{aligned}
\mathbb{E}_{\theta}\left[u_{i}\left(\left(Q\left(\theta_{H}, k_{L}\right) Q\left(\theta_{L}, k_{L}\right), Q\left(\theta_{H}, k_{H}\right) Q\left(\theta_{L}, k_{H}\right)\right), \theta_{i}\right)\right] \\
>\mathbb{E}_{\theta}\left[u_{i}\left(\left(Q\left(\theta_{H}, k_{L}\right) Q\left(\theta_{L}, k_{H}\right), Q\left(\theta_{H}, k_{H}\right) Q\left(\theta_{L}, k_{H}\right)\right), \theta_{i}\right)\right] \\
\Leftrightarrow 1>1-\frac{1}{2}\left(1-\mu^{2}\right)>0 \Leftrightarrow 0>-\frac{1}{2}\left(1-\mu^{2}\right)
\end{aligned}
$$

and

$$
\begin{aligned}
& \mathbb{E}_{\theta}\left[u_{i}\left(\left(Q\left(\theta_{H}, k_{L}\right) Q\left(\theta_{L}, k_{L}\right), Q\left(\theta_{H}, k_{H}\right) Q\left(\theta_{L}, k_{H}\right)\right), \theta_{i}\right)\right] \\
&>\mathbb{E}_{\theta}\left[u_{i}\left(\left(Q\left(\theta_{H}, k_{H}\right) Q\left(\theta_{L}, k_{L}\right), Q\left(\theta_{H}, k_{H}\right) Q\left(\theta_{L}, k_{H}\right)\right), \theta_{i}\right)\right] \\
& \Leftrightarrow 1>1-\frac{\mu^{2}}{2}>0 \Leftrightarrow 0>-\frac{\mu^{2}}{2} .
\end{aligned}
$$

Conditions (20) and (21) are trivially satisfied for $\mu \in(0,1)$.

Therefore, I have shown that, irrespective of player $j$ 's strategy, player $i$ 's best response is $\sigma_{i}\left(\theta_{i}\right)=\left(Q\left(\theta_{H}, k_{L}\right) Q\left(\theta_{L}, k_{L}\right)\right)$. As $i, j \in \mathscr{I}$ were assigned arbitrarily and the players' payoffs are symmetric this implies that the strategy profile $\sigma^{*}=\left(Q\left(\theta_{H}, k_{L}\right) Q\left(\theta_{L}, k_{L}\right), Q\left(\theta_{H}, k_{L}\right) Q\left(\theta_{L}, k_{L}\right)\right)$ is the unique pure strategy Bayes-Nash equilibrium of the game in Figure 4.

Suppose now that $\mathscr{K}=\left\{k_{H}\right\}$. The initial strategic form games for this case are illustrated 
in Figure 5. Once again I use the procedure laid out in Subsection 4.1 to derive $\mathbb{E}_{\theta}\left[u_{i}\left(\sigma, \theta_{i}\right)\right]$

2

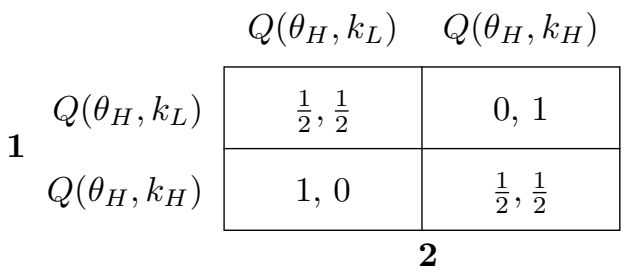

$Q\left(\theta_{H}, k_{L}\right) \quad Q\left(\theta_{H}, k_{H}\right)$

1

\begin{tabular}{c|c|c|}
\multicolumn{1}{c}{$Q\left(\theta_{H}, k_{L}\right)$} & $Q\left(\theta_{H}, k_{H}\right)$ \\
\cline { 2 - 3 }$Q\left(\theta_{L}, k_{L}\right)$ & 0,1 & 0,1 \\
\cline { 2 - 3 }$Q\left(\theta_{L}, k_{H}\right)$ & 1,0 & 0,1 \\
\cline { 2 - 3 } & &
\end{tabular}

2

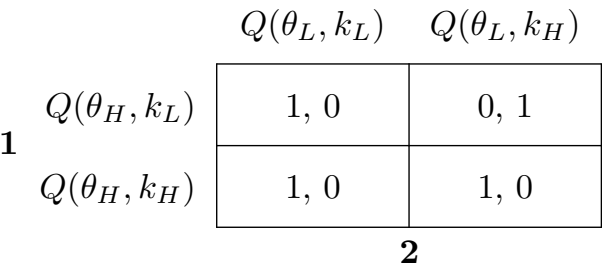

$Q\left(\theta_{L}, k_{L}\right) \quad Q\left(\theta_{L}, k_{H}\right)$

1

\begin{tabular}{|c|c|c|}
\cline { 2 - 3 }$Q\left(\theta_{L}, k_{L}\right)$ & $\frac{1}{2}, \frac{1}{2}$ & 0,1 \\
\cline { 2 - 3 }$Q\left(\theta_{L}, k_{H}\right)$ & 1,0 & $\frac{1}{2}, \frac{1}{2}$ \\
\hline
\end{tabular}

Figure 5: Strategic Form Games when $\mathscr{K}=\left\{k_{H}\right\}$.

for each possible strategy profile. This leads to the Bayesian strategic form of the game that is illustrated in Figure 6.

Analogously to earlier in the proof, I will now show that, for each $i \in \mathscr{I}$, the strategy $\sigma_{i}\left(\theta_{i}\right)=\left(Q\left(\theta_{H}, k_{H}\right) Q\left(\theta_{L}, k_{H}\right)\right)$ is a best response to any strategy of player $j \in \mathscr{I} \backslash\{i\}$. Suppose first that player $j$ uses the strategy $\sigma_{j}\left(\theta_{j}\right)=\left(Q\left(\theta_{H}, k_{L}\right) Q\left(\theta_{L}, k_{L}\right)\right)$ then conditions (20) and (21) imply that player $i$ 's strategy is a best response. Supposing now that player $j$ uses the strategy $\sigma_{j}\left(\theta_{j}\right)=\left(Q\left(\theta_{H}, k_{L}\right) Q\left(\theta_{L}, k_{H}\right)\right)$ then conditions (17)-(19) imply that player $i$ 's strategy is a best response. Next, suppose that player $j$ uses the strategy $\sigma_{j}\left(\theta_{j}\right)=\left(Q\left(\theta_{H}, k_{H}\right) Q\left(\theta_{L}, k_{L}\right)\right)$ then conditions (14)-(16) imply that player $i$ 's strategy is a best response. Finally, suppose that player $j$ uses the strategy $\sigma_{j}\left(\theta_{j}\right)=\left(Q\left(\theta_{H}, k_{H}\right) Q\left(\theta_{L}, k_{H}\right)\right)$ then conditions (12) and (13) imply that player $i$ 's strategy is a best response.

Therefore, I have shown that, irrespective of player $j$ 's strategy, player $i$ 's best response is $\sigma_{i}\left(\theta_{i}\right)=\left(Q\left(\theta_{H}, k_{H}\right) Q\left(\theta_{L}, k_{H}\right)\right)$. As $i, j \in \mathscr{I}$ were assigned arbitrarily and the players' payoffs are symmetric this implies that the strategy profile $\sigma^{*}=\left(Q\left(\theta_{H}, k_{H}\right) Q\left(\theta_{L}, k_{H}\right), Q\left(\theta_{H}, k_{H}\right) Q\left(\theta_{L}, k_{H}\right)\right)$ is the unique pure strategy Bayes-Nash equilibrium of the game in Figure 6.

I now consider the case when Assumption 1 holds but Assumption 2 does not. It will suffice to analyse the equilibrium when $\mathscr{K}=\left\{k_{L}\right\}$ as the strategic form games when $\mathscr{K}=$ $\left\{k_{H}\right\}$ are unchanged from Figure 5 and, hence, from the Bayesian strategic form in Figure 6. This implies that the strategy profile $\sigma^{*}=\left(Q\left(\theta_{H}, k_{H}\right) Q\left(\theta_{L}, k_{H}\right), Q\left(\theta_{H}, k_{H}\right) Q\left(\theta_{L}, k_{H}\right)\right)$ remains the unique pure strategy Bayes-Nash equilibrium. Therefore, supposing that $\mathscr{K}=\left\{k_{L}\right\}$, the initial strategic form games are illustrated in Figure 7. Relative to Figure 3, the changes in payoffs from relaxing Assumption 2 occur at the action profiles $\sigma=\left(Q\left(\theta_{H}, k_{H}\right), Q\left(\theta_{L}, k_{L}\right)\right)$ and $\sigma=\left(Q\left(\theta_{L}, k_{L}\right), Q\left(\theta_{H}, k_{H}\right)\right)$. The Bayesian strategic form that incorporates these changes is illustrated in Figure 8 .

I will now show that, for each $i \in \mathscr{I}$, the strategy $\sigma_{i}\left(\theta_{i}\right)=\left(Q\left(\theta_{H}, k_{L}\right) Q\left(\theta_{L}, k_{L}\right)\right)$ is a best 


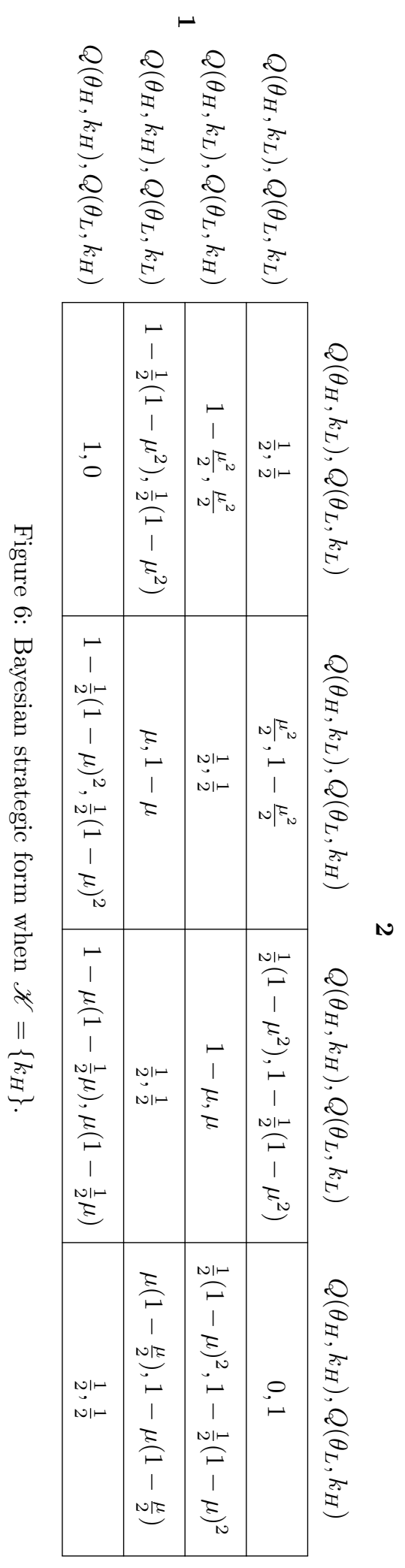



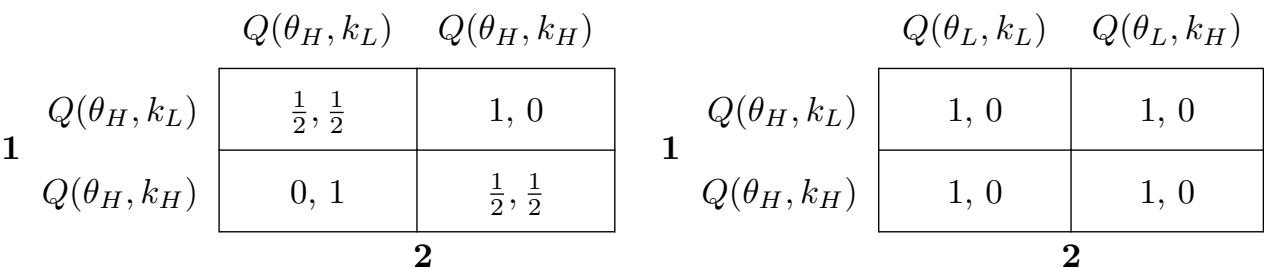

\begin{tabular}{|c|c|c|}
\hline & $Q\left(\theta_{H}, k_{L}\right)$ & $Q\left(\theta_{H}, k_{H}\right)$ \\
\hline$Q\left(\theta_{L}, k_{L}\right)$ & 0,1 & 0,1 \\
\hline$Q\left(\theta_{L}, k_{H}\right)$ & 0,1 & 0,1 \\
\hline
\end{tabular}

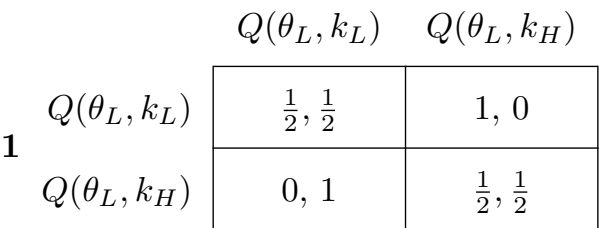

Figure 7: Strategic form games when $\mathscr{K}=\left\{k_{L}\right\}$ and Assumption 2 does not hold.

response to any strategy of player $j \in \mathscr{I} \backslash\{i\}$. Suppose player $j$ uses the strategy $\sigma_{j}\left(\theta_{j}\right)=$ $\left(Q\left(\theta_{H}, k_{L}\right) Q\left(\theta_{L}, k_{L}\right)\right)$, then, player $i$ 's strategy is a best response by conditions (12) and (17), and if

$$
\begin{aligned}
\mathbb{E}_{\theta}\left[u_{i}\left(\left(Q\left(\theta_{H}, k_{L}\right) Q\left(\theta_{L}, k_{L}\right), Q\left(\theta_{H}, k_{L}\right) Q\left(\theta_{L}, k_{L}\right)\right), \theta_{i}\right)\right] & \\
>\mathbb{E}_{\theta}\left[u_{i}\left(\left(Q\left(\theta_{H}, k_{H}\right) Q\left(\theta_{L}, k_{H}\right), Q\left(\theta_{H}, k_{L}\right) Q\left(\theta_{L}, k_{L}\right)\right), \theta_{i}\right)\right] & \Leftrightarrow \frac{1}{2}>\mu(1-\mu) .
\end{aligned}
$$

Condition (22) holds as $\mu(1-\mu)$ is a strictly concave function that is maximised at $\mu^{*}=1 / 2$; that is, $\mu^{*}=\arg \max _{\mu} \mu(1-\mu)$. This optimum yields a maximum value $V\left(\mu^{*}\right)=1 / 4<1 / 2$. Suppose now that player $j$ uses the strategy $\sigma_{j}\left(\theta_{j}\right)=\left(Q\left(\theta_{H}, k_{L}\right) Q\left(\theta_{L}, k_{H}\right)\right)$ then player $i$ 's strategy is a best response by conditions (14)-(16). Next, suppose that player $j$ uses the strategy $\sigma_{j}\left(\theta_{j}\right)=\left(Q\left(\theta_{H}, k_{H}\right) Q\left(\theta_{L}, k_{L}\right)\right)$ then player $i$ 's strategy is a best response by conditions (17) and (18), and if

$$
\begin{aligned}
& \mathbb{E}_{\theta}\left[u_{i}\left(\left(Q\left(\theta_{H}, k_{L}\right) Q\left(\theta_{L}, k_{L}\right), Q\left(\theta_{H}, k_{H}\right) Q\left(\theta_{L}, k_{L}\right)\right), \theta_{i}\right)\right] \\
&>\mathbb{E}_{\theta}\left[u_{i}\left(\left(Q\left(\theta_{H}, k_{H}\right) Q\left(\theta_{L}, k_{H}\right), Q\left(\theta_{H}, k_{H}\right) Q\left(\theta_{L}, k_{L}\right)\right), \theta_{i}\right)\right] \\
& \Leftrightarrow 1-\frac{1}{2}\left(1-\mu^{2}\right)>\mu\left(1-\frac{\mu}{2}\right) \Leftrightarrow \frac{1}{2}>\mu(1-\mu) .
\end{aligned}
$$

Condition (23) is equivalent to condition (22) and, therefore, holds. Finally, suppose that player 


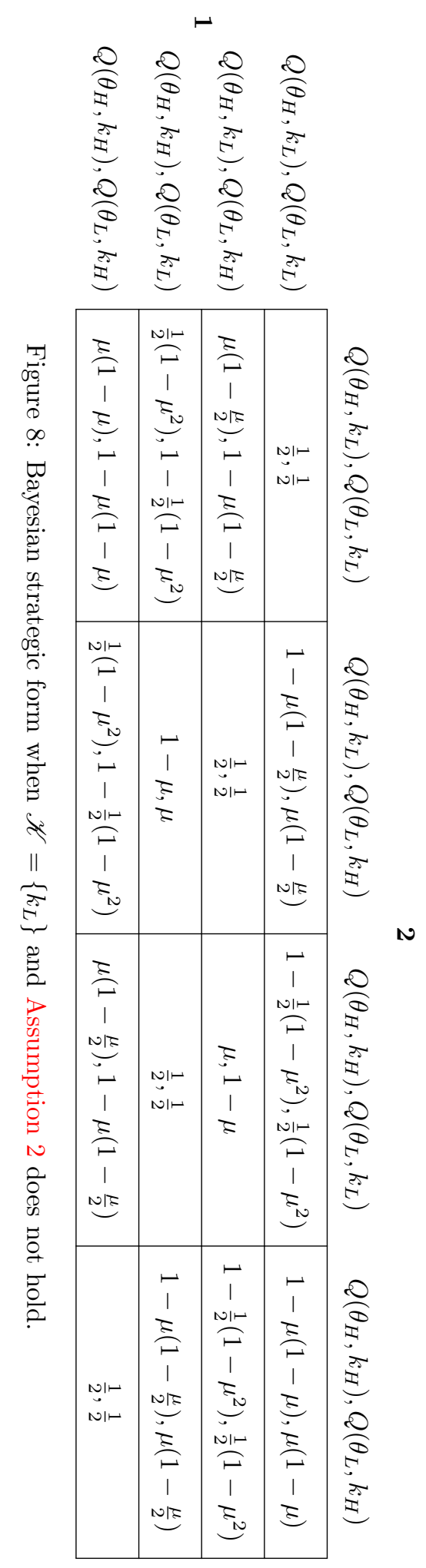


$j$ uses the strategy $\sigma_{j}\left(\theta_{j}\right)=\left(Q\left(\theta_{H}, k_{H}\right) Q\left(\theta_{L}, k_{H}\right)\right)$ then player $i$ 's strategy is a best response if

$$
\begin{aligned}
& \mathbb{E}_{\theta}\left[u_{i}\left(\left(Q\left(\theta_{H}, k_{L}\right) Q\left(\theta_{L}, k_{L}\right), Q\left(\theta_{H}, k_{H}\right) Q\left(\theta_{L}, k_{H}\right)\right), \theta_{i}\right)\right] \\
& >\mathbb{E}_{\theta}\left[u_{i}\left(\left(Q\left(\theta_{H}, k_{L}\right) Q\left(\theta_{L}, k_{H}\right), Q\left(\theta_{H}, k_{H}\right) Q\left(\theta_{L}, k_{H}\right)\right), \theta_{i}\right)\right] \\
& \Leftrightarrow 1-\mu(1-\mu)>1-\frac{1}{2}\left(1-\mu^{2}\right) \Leftrightarrow \frac{1}{2}>\mu\left(1-\frac{\mu}{2}\right),
\end{aligned}
$$

$$
\begin{aligned}
& \mathbb{E}_{\theta}\left[u_{i}\left(\left(Q\left(\theta_{H}, k_{L}\right) Q\left(\theta_{L}, k_{L}\right), Q\left(\theta_{H}, k_{H}\right) Q\left(\theta_{L}, k_{H}\right)\right), \theta_{i}\right)\right] \\
& >\mathbb{E}_{\theta}\left[u_{i}\left(\left(Q\left(\theta_{H}, k_{H}\right) Q\left(\theta_{L}, k_{L}\right), Q\left(\theta_{H}, k_{H}\right) Q\left(\theta_{L}, k_{H}\right)\right), \theta_{i}\right)\right] \\
& \Leftrightarrow 1-\mu(1-\mu)>1-\mu\left(1-\frac{\mu}{2}\right) \Leftrightarrow \mu\left(1-\frac{\mu}{2}\right)>\mu(1-\mu)
\end{aligned}
$$

and

$$
\begin{aligned}
& \mathbb{E}_{\theta}\left[u_{i}\left(\left(Q\left(\theta_{H}, k_{L}\right) Q\left(\theta_{L}, k_{L}\right), Q\left(\theta_{H}, k_{H}\right) Q\left(\theta_{L}, k_{H}\right)\right), \theta_{i}\right)\right] \\
&>\mathbb{E}_{\theta}\left[u_{i}\left(\left(Q\left(\theta_{H}, k_{H}\right) Q\left(\theta_{L}, k_{H}\right), Q\left(\theta_{H}, k_{H}\right) Q\left(\theta_{L}, k_{H}\right)\right), \theta_{i}\right)\right] \\
& \Leftrightarrow 1-\mu(1-\mu)>\frac{1}{2} \Leftrightarrow \frac{1}{2}>\mu(1-\mu) .
\end{aligned}
$$

Condition (24) holds by (14), condition (25) holds for any $\mu>0$ and condition (26) holds by (22).

Therefore, I have shown that, irrespective of player $j$ 's strategy, player $i$ 's best response is $\sigma_{i}\left(\theta_{i}\right)=\left(Q\left(\theta_{H}, k_{L}\right) Q\left(\theta_{L}, k_{L}\right)\right)$. As $i, j \in \mathscr{I}$ were assigned arbitrarily and the players' payoffs are symmetric this implies that the strategy profile $\sigma^{*}=\left(Q\left(\theta_{H}, k_{L}\right) Q\left(\theta_{L}, k_{L}\right), Q\left(\theta_{H}, k_{L}\right) Q\left(\theta_{L}, k_{L}\right)\right)$ is the unique pure strategy Bayes-Nash equilibrium of the game in Figure 8. This completes the proof of Proposition 2.

Proof of Proposition 3. Given that Assumption 1 and Assumption 2 hold, the supposition that $\operatorname{Pr}\left(k=k_{L}\right)=\operatorname{Pr}(k=1)$ implies that the Bayesian strategic form game in Figure 11 yields the Bayesian strategic form illustrated in Figure 9. The expected payoffs $f:(0,1) \rightarrow\left(\frac{1}{2}, \frac{5}{8}\right]$ and $g:(0,1) \rightarrow\left[\frac{3}{8}, \frac{1}{2}\right)$ given in Figure 9 are defined by

$$
f(\mu):=\frac{1}{2}+\frac{\mu}{2}(1-\mu)>\frac{1}{2}>\frac{1}{2}+\frac{\mu}{2}(\mu-1):=g(\mu) .
$$

I will now show that there does not exist mutually consistent best responses in pure strategies. That is, there is no pure strategy profile $\sigma=\left(\sigma_{i}\left(\theta_{i}\right), \sigma_{j}\left(\theta_{j}\right)\right)$ for $i, j \in \mathscr{I}$ with $i \neq j$ such that player $i$ 's strategy $\sigma_{i}\left(\theta_{i}\right)$ is a best response to player $j \in \mathscr{I} \backslash\{i\}$ 's strategy $\sigma_{j}\left(\theta_{j}\right)$ and vice versa.

Suppose first that player $j$ uses the strategy $\sigma_{j}\left(\theta_{j}\right)=\left(Q\left(\theta_{H}, k_{L}\right) Q\left(\theta_{L}, k_{L}\right)\right)$, then, player $i$ 's best response is $\sigma_{i}\left(\theta_{i}\right)=\left(Q\left(\theta_{H}, k_{L}\right) Q\left(\theta_{L}, k_{H}\right)\right)$. Suppose now that player $j$ uses the strategy $\sigma_{j}\left(\theta_{j}\right)=\left(Q\left(\theta_{H}, k_{L}\right) Q\left(\theta_{L}, k_{H}\right)\right)$, then, player $i$ 's best response is $\sigma_{i}\left(\theta_{i}\right)=\left(Q\left(\theta_{H}, k_{H}\right) Q\left(\theta_{L}, k_{H}\right)\right)$. Next, suppose that player $j$ uses the strategy $\sigma_{j}\left(\theta_{j}\right)=\left(Q\left(\theta_{H}, k_{H}\right) Q\left(\theta_{L}, k_{L}\right)\right)$, then, player $i$ 's 


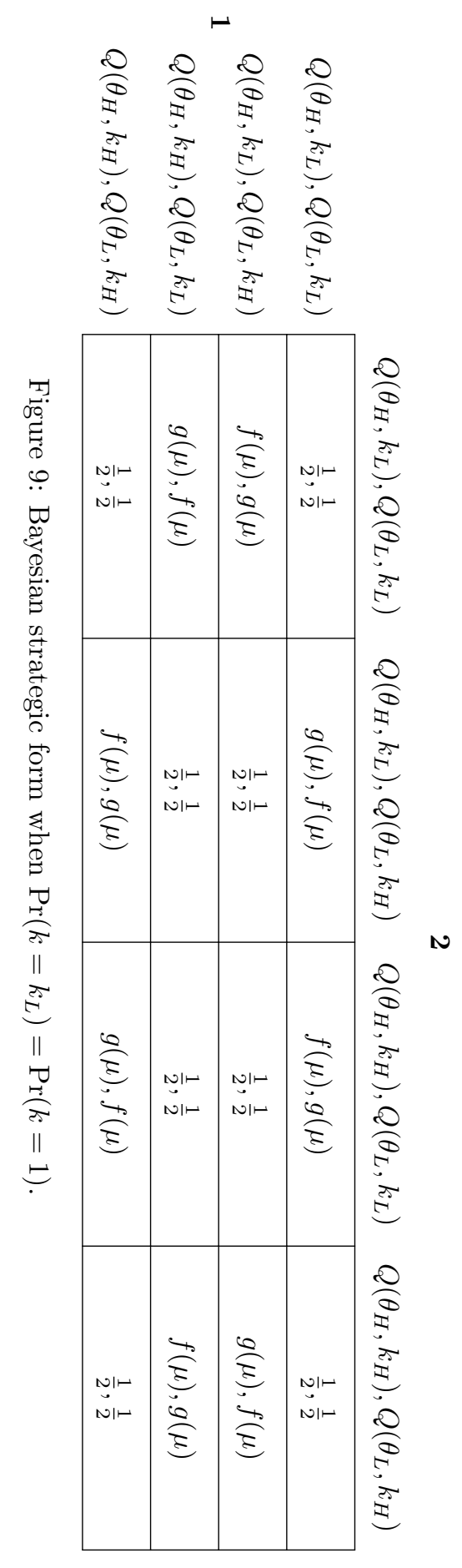


best response is $\sigma_{i}\left(\theta_{i}\right)=\left(Q\left(\theta_{H}, k_{L}\right) Q\left(\theta_{L}, k_{L}\right)\right)$. Finally, suppose that player $j$ uses the strategy $\sigma_{j}\left(\theta_{j}\right)=\left(Q\left(\theta_{H}, k_{H}\right) Q\left(\theta_{L}, k_{H}\right)\right)$, then, player $i$ 's best response is $\sigma_{i}\left(\theta_{i}\right)=\left(Q\left(\theta_{H}, k_{H}\right) Q\left(\theta_{L}, k_{L}\right)\right)$.

Therefore, I have shown that, for any $\operatorname{Pr}\left(\theta_{i}=\theta_{H}\right)=\mu$, there are no mutually consistent best responses in pure strategies and, hence, there are no pure strategy Bayes-Nash equilibria. This completes the proof of Proposition 3.

Proof of Corollary 1. Given that Assumption 1 holds but Assumption 2 does not, the supposition that $\operatorname{Pr}\left(k=k_{L}\right)=\operatorname{Pr}(k=1)$ implies that the Bayesian strategic form game given in Figure 11 yields the Bayesian strategic form illustrated in Figure 10, where $f(\mu)$ and $g(\mu)$ are defined as in (27).

I will now show that, by iterated elimination of weakly dominated strategies, the unique pure strategy Bayes-Nash equilibrium is $\sigma^{*}=\left(Q\left(\theta_{H}, k_{H}\right) Q\left(\theta_{L}, k_{H}\right), Q\left(\theta_{H}, k_{H}\right) Q\left(\theta_{L}, k_{H}\right)\right)$. That is, after eliminating strategy profiles in which players employ weakly dominated strategies, the strategy $\sigma_{i}\left(\theta_{i}\right)=\left(Q\left(\theta_{H}, k_{H}\right) Q\left(\theta_{L}, k_{H}\right)\right)$ for $i \in \mathscr{I}$ is a best response to any strategy of player $j \in \mathscr{I} \backslash\{i\}$.

Suppose first that player $j$ uses the strategy $\sigma_{j}\left(\theta_{j}\right)=\left(Q\left(\theta_{H}, k_{L}\right) Q\left(\theta_{L}, k_{L}\right)\right)$ then both $\sigma_{i}\left(\theta_{i}\right)=\left(Q\left(\theta_{H}, k_{H}\right) Q\left(\theta_{L}, k_{L}\right)\right)$ and $\sigma_{i}\left(\theta_{i}\right)=\left(Q\left(\theta_{H}, k_{H}\right) Q\left(\theta_{L}, k_{H}\right)\right)$ are best responses for player $i$. Suppose now that player $j$ uses the strategy $\sigma_{j}\left(\theta_{j}\right)=\left(Q\left(\theta_{H}, k_{L}\right) Q\left(\theta_{L}, k_{H}\right)\right)$ then player $i$ 's unique best response is the strategy $\sigma_{i}\left(\theta_{i}\right)=\left(Q\left(\theta_{H}, k_{H}\right) Q\left(\theta_{L}, k_{H}\right)\right)$. Next, suppose that player $j$ uses the strategy $\sigma_{j}\left(\theta_{j}\right)=\left(Q\left(\theta_{H}, k_{H}\right) Q\left(\theta_{L}, k_{L}\right)\right)$ then all of player $i$ 's strategies constitute best responses. Finally, suppose that player $j$ uses the strategy $\sigma_{j}\left(\theta_{j}\right)=\left(Q\left(\theta_{H}, k_{H}\right) Q\left(\theta_{L}, k_{H}\right)\right)$ then both $\sigma_{i}\left(\theta_{i}\right)=\left(Q\left(\theta_{H}, k_{H}\right) Q\left(\theta_{L}, k_{L}\right)\right)$ and $\sigma_{i}\left(\theta_{i}\right)=\left(Q\left(\theta_{H}, k_{H}\right) Q\left(\theta_{L}, k_{H}\right)\right)$ are best responses for player $i$.

The set of pure strategy Bayes-Nash equilibria is therefore

$$
\begin{aligned}
& \left\{\left(Q\left(\theta_{H}, k_{H}\right) Q\left(\theta_{L}, k_{L}\right), Q\left(\theta_{H}, k_{H}\right) Q\left(\theta_{L}, k_{L}\right)\right),\left(Q\left(\theta_{H}, k_{H}\right) Q\left(\theta_{L}, k_{H}\right), Q\left(\theta_{H}, k_{H}\right) Q\left(\theta_{L}, k_{L}\right)\right),\right. \\
& \left.\quad\left(Q\left(\theta_{H}, k_{H}\right) Q\left(\theta_{L}, k_{L}\right), Q\left(\theta_{H}, k_{H}\right) Q\left(\theta_{L}, k_{H}\right)\right),\left(Q\left(\theta_{H}, k_{H}\right) Q\left(\theta_{L}, k_{H}\right), Q\left(\theta_{H}, k_{H}\right) Q\left(\theta_{L}, k_{H}\right)\right)\right\} .
\end{aligned}
$$

Note that the strategy $\sigma_{i}\left(\theta_{i}\right)=\left(Q\left(\theta_{H}, k_{H}\right) Q\left(\theta_{L}, k_{L}\right)\right)$ is weakly dominated by the strategy $\sigma_{i}\left(\theta_{i}\right)=\left(Q\left(\theta_{H}, k_{H}\right) Q\left(\theta_{L}, k_{H}\right)\right)$ for each $i \in \mathscr{I}$. This implies that, by iterated elimination of weakly dominated strategies, the unique pure strategy Bayes-Nash equilibrium is the strategy profile $\sigma_{\text {IEoWDS }}^{*}=\left(Q\left(\theta_{H}, k_{H}\right) Q\left(\theta_{L}, k_{H}\right), Q\left(\theta_{H}, k_{H}\right) Q\left(\theta_{L}, k_{H}\right)\right)$. This completes the proof of Corollary 1.

Proof of Proposition 4. Suppose each player $i \in \mathscr{I}$ employs a mixed strategy $\hat{\sigma}\left(\theta_{i}\right) \in \Delta(\mathscr{T})$ that randomises over her pure strategies with probabilities

$$
\begin{aligned}
& \operatorname{Pr}\left[\sigma_{i}\left(\theta_{i}\right)=\left(Q\left(\theta_{H}, k_{L}\right) Q\left(\theta_{L}, k_{L}\right)\right)\right]:=p \in[0,1], \\
& \operatorname{Pr}\left[\sigma_{i}\left(\theta_{i}\right)=\left(Q\left(\theta_{H}, k_{L}\right) Q\left(\theta_{L}, k_{H}\right)\right)\right]:=q \in[0,1], \\
& \operatorname{Pr}\left[\sigma_{i}\left(\theta_{i}\right)=\left(Q\left(\theta_{H}, k_{H}\right) Q\left(\theta_{L}, k_{L}\right)\right)\right]:=r \in[0,1]
\end{aligned}
$$




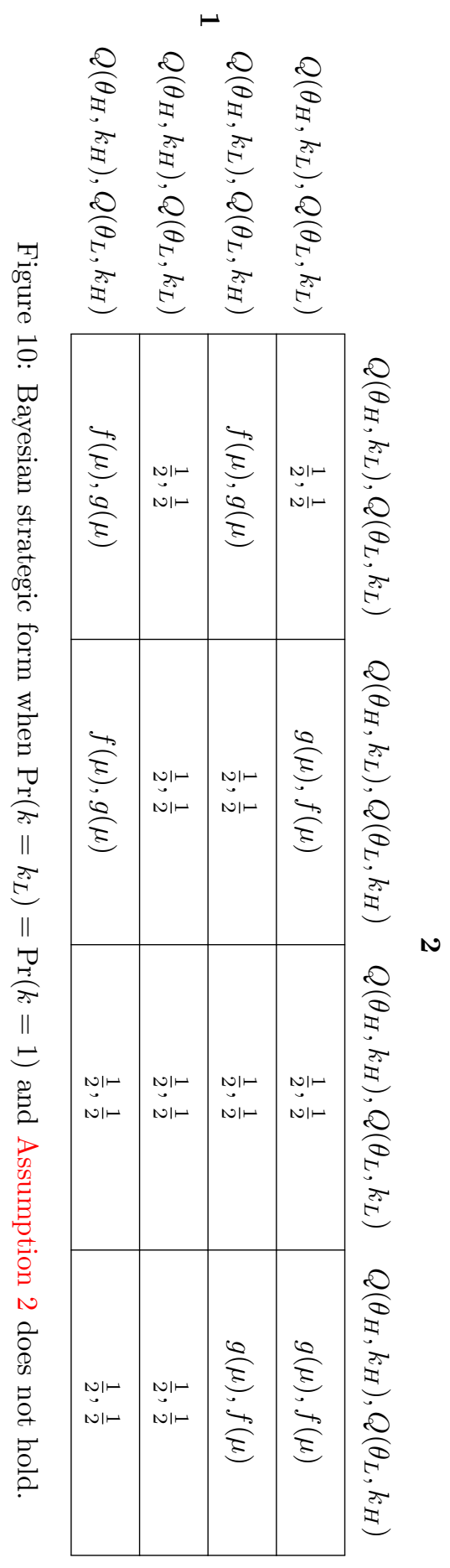


and

$$
\operatorname{Pr}\left[\sigma_{i}\left(\theta_{i}\right)=\left(Q\left(\theta_{H}, k_{H}\right) Q\left(\theta_{L}, k_{H}\right)\right)\right]:=1-p-q-r .
$$

Player $i$ then chooses a vector $(p, q, r) \in[0,1]^{3}$ to make player $j \in \mathscr{I} \backslash\{I\}$ indifferent between her pure strategies, subject to the constraint that $p+q+r \leq 1$. For player $j$ to be indifferent between her pure strategies the following indifference conditions must hold

$$
\begin{aligned}
& \mathbb{E}_{\theta, k}\left[u_{j}\left(\left(Q\left(\theta_{H}, k_{L}\right) Q\left(\theta_{L}, k_{L}\right), \hat{\sigma}\left(\theta_{i}\right)\right), \theta_{j}\right)\right]=\mathbb{E}_{\theta, k}\left[u_{j}\left(\left(Q\left(\theta_{H}, k_{L}\right) Q\left(\theta_{L}, k_{H}\right), \hat{\sigma}\left(\theta_{i}\right)\right), \theta_{j}\right)\right], \\
& \mathbb{E}_{\theta, k}\left[u_{j}\left(\left(Q\left(\theta_{H}, k_{L}\right) Q\left(\theta_{L}, k_{H}\right), \hat{\sigma}\left(\theta_{i}\right)\right), \theta_{j}\right)\right]=\mathbb{E}_{\theta, k}\left[u_{j}\left(\left(Q\left(\theta_{H}, k_{H}\right) Q\left(\theta_{L}, k_{L}\right), \hat{\sigma}\left(\theta_{i}\right)\right), \theta_{j}\right)\right]
\end{aligned}
$$

and

$$
\mathbb{E}_{\theta, k}\left[u_{j}\left(\left(Q\left(\theta_{H}, k_{H}\right) Q\left(\theta_{L}, k_{L}\right), \hat{\sigma}\left(\theta_{i}\right)\right), \theta_{j}\right)\right]=\mathbb{E}_{\theta, k}\left[u_{j}\left(\left(Q\left(\theta_{H}, k_{H}\right) Q\left(\theta_{L}, k_{H}\right), \hat{\sigma}\left(\theta_{i}\right)\right), \theta_{j}\right)\right] .
$$

The three indifference conditions (28)-(30) are sufficient to solve for the three unknown elements of the vector $(p, q, r)$. By Figure 9, condition (28) is equivalent to

$$
\begin{aligned}
\frac{p}{2}+q\left[\frac{1}{2}+\frac{\mu}{2}(\mu-1)\right] & +r\left[\frac{1}{2}+\frac{\mu}{2}(1-\mu)\right]+\frac{1}{2}(1-p-q-r) \\
& =p\left[\frac{1}{2}+\frac{\mu}{2}(1-\mu)\right]+\frac{q}{2}+\frac{r}{2}+(1-p-q-r)\left[\frac{1}{2}+\frac{\mu}{2}(\mu-1)\right],
\end{aligned}
$$

whilst condition (29) is satisfied if

$$
\begin{aligned}
p\left[\frac{1}{2}+\frac{\mu}{2}(1-\mu)\right] & +\frac{q}{2}+\frac{r}{2}+(1-p-q-r)\left[\frac{1}{2}+\frac{\mu}{2}(\mu-1)\right] \\
& =p\left[\frac{1}{2}+\frac{\mu}{2}(\mu-1)\right]+\frac{q}{2}+\frac{r}{2}+(1-p-q-r)\left[\frac{1}{2}+\frac{\mu}{2}(1-\mu)\right] .
\end{aligned}
$$

Finally, condition (30) will hold if

$$
\begin{aligned}
p\left[\frac{1}{2}+\frac{\mu}{2}(\mu-1)\right]+\frac{q}{2} & +\frac{r}{2}+(1-p-q-r)\left[\frac{1}{2}+\frac{\mu}{2}(1-\mu)\right] \\
& =q\left[\frac{1}{2}+\frac{\mu}{2}(1-\mu)\right]+r\left[\frac{1}{2}+\frac{\mu}{2}(\mu-1)\right]+\frac{1}{2}(1-p-q-r) .
\end{aligned}
$$

I now suppose that $q^{*}=r^{*}=0$ and demonstrate that this condition is sufficient to identify a mixed strategy Bayes-Nash equilibrium; that is, $q^{*}=r^{*}=0$ pins down a unique $p^{*}>0$ that satisfies (31)-(33). First, substituting $q^{*}=r^{*}=0$ into (31) yields

$$
p\left[\frac{1}{2}+\frac{\mu}{2}(1-\mu)\right]-p\left[\frac{1}{2}+\frac{\mu}{2}(\mu-1)\right]+\left[\frac{1}{2}+\frac{\mu}{2}(\mu-1)\right]=\frac{1}{2},
$$


which simplifies to

$$
p^{*}=\frac{\frac{1}{2}-\left[\frac{1}{2}+\frac{\mu}{2}(\mu-1)\right]}{\left[\frac{1}{2}+\frac{\mu}{2}(1-\mu)\right]-\left[\frac{1}{2}+\frac{\mu}{2}(\mu-1)\right]}=\frac{\frac{\mu}{2}(1-\mu)}{\frac{\mu}{2}(1-\mu)+\frac{\mu}{2}(1-\mu)}=\frac{\mu(1-\mu)}{2 \mu(1-\mu)}=\frac{1}{2} .
$$

Next, substituting $q^{*}=r^{*}=0$ into (32) yields

$$
2 p\left[\frac{1}{2}+\frac{\mu}{2}(1-\mu)\right]-2 p\left[\frac{1}{2}+\frac{\mu}{2}(\mu-1)\right]=\left[\frac{1}{2}+\frac{\mu}{2}(1-\mu)\right]-\left[\frac{1}{2}+\frac{\mu}{2}(\mu-1)\right],
$$

which simplifies to

$$
p^{*}=\frac{\left[\frac{1}{2}+\frac{\mu}{2}(1-\mu)\right]-\left[\frac{1}{2}+\frac{\mu}{2}(\mu-1)\right]}{2\left[\left[\frac{1}{2}+\frac{\mu}{2}(1-\mu)\right]-\left[\frac{1}{2}+\frac{\mu}{2}(\mu-1)\right]\right]}=\frac{1}{2} .
$$

Finally, substituting $q^{*}=r^{*}=0$ into (33) yields

$$
p\left[\frac{1}{2}+\frac{\mu}{2}(\mu-1)\right]-p\left[\frac{1}{2}+\frac{\mu}{2}(1-\mu)\right]+\left[\frac{1}{2}+\frac{\mu}{2}(1-\mu)\right]=\frac{1}{2},
$$

which simplifies to

$$
p^{*}=\frac{\frac{1}{2}-\left[\frac{1}{2}+\frac{\mu}{2}(1-\mu)\right]}{\left[\frac{1}{2}+\frac{\mu}{2}(\mu-1)\right]-\left[\frac{1}{2}+\frac{\mu}{2}(1-\mu)\right]}=\frac{\frac{\mu}{2}(\mu-1)}{\frac{\mu}{2}(\mu-1)+\frac{\mu}{2}(\mu-1)}=\frac{\mu(\mu-1)}{2 \mu(\mu-1)}=\frac{1}{2} .
$$

Note that equations (34)-(36) are independent of $\operatorname{Pr}\left(\theta_{i}=\theta_{H}\right)$. Therefore, the vector $\left(p^{*}, q^{*}, r^{*}\right)=$ $\left(\frac{1}{2}, 0,0\right)$ makes player $j$ indifferent between her pure strategies for all $\operatorname{Pr}\left(\theta_{i}=\theta_{H}\right)$. As player $i$ and $j$ are symmetric, and were arbitrarily assigned, this vector constitutes a mixed strategy Bayes-Nash equilibrium.

This equilibrium is equivalent to each player $i \in \mathscr{I}$ using the strategy $\hat{\sigma}_{i}\left(\theta_{i}\right)=\mathbb{E}_{k}\left[Q\left(\theta_{i}, k\right)\right]$ as player $i$ 's expected performance, when $\operatorname{Pr}\left(k=k_{L}\right)=\operatorname{Pr}\left(k=k_{H}\right)$, is equal to

$$
\begin{aligned}
\mathbb{E}_{k}\left[Q\left(\theta_{i}, k\right)\right] & =\operatorname{Pr}\left(k=k_{L}\right) Q\left(\theta_{i}, k_{L}\right)+\operatorname{Pr}\left(k=k_{H}\right) Q\left(\theta_{i}, k_{H}\right), \\
& =\operatorname{Pr}\left(k=k_{L}\right) \theta_{i}+\operatorname{Pr}\left(k=k_{H}\right)\left(\theta_{i}+k_{H}\right), \\
& =\frac{\theta_{i}}{2}+\frac{k_{H}}{2}\left(\theta_{i}+1\right)=\theta_{i}+\frac{k_{H}}{2} .
\end{aligned}
$$

Moreover, the mixed strategy determined by the vector $\left(p^{*}, q^{*}, r^{*}\right)=\left(\frac{1}{2}, 0,0\right)$ is

$$
\begin{aligned}
\hat{\sigma}\left(\theta_{i}\right) & =p^{*} Q\left(\theta_{i}, k_{L}\right)+\left(1-p^{*}-q^{*}-r^{*}\right) Q\left(\theta_{i}, k_{H}\right), \\
& =\frac{\theta_{i}}{2}+\left(1-\frac{1}{2}\right)\left(\theta_{i}+k_{H}\right)=\theta_{i}+\frac{k_{H}}{2} .
\end{aligned}
$$

Hence, as (37) is equivalent to (37), player $i$ 's mixed strategy $\hat{\sigma}_{i}\left(\theta_{i}\right)$ is equivalent to using a strategy based upon her expected performance $\mathbb{E}_{k}\left[Q\left(\theta_{i}, k\right)\right]$. I have assumed that $Q(\theta, k)=$ $\theta+k$ for exposition, but the result holds for any $Q: \Theta \times \mathscr{K}$ that satisfies Assumption 1 and Assumption 2.

I now suppose that $p^{*}=0$ and demonstrate that this condition is sufficient to identify a 
mixed strategy Bayes-Nash equilibrium; that is, $p^{*}=0$ pins down a unique $q^{*}>0$ and $r^{*}>0$ that satisfy (31)-(33). First, substituting $p^{*}=0$ into (31) yields

$$
\begin{aligned}
q\left[\frac{1}{2}+\frac{\mu}{2}(\mu-1)\right] & +r\left[\frac{1}{2}+\frac{\mu}{2}(1-\mu)\right]+\frac{1}{2}-\frac{q}{2}-\frac{r}{2} \\
& =\frac{q}{2}+\frac{r}{2}+\left[\frac{1}{2}+\frac{\mu}{2}(\mu-1)\right]-q\left[\frac{1}{2}+\frac{\mu}{2}(\mu-1)\right]-r\left[\frac{1}{2}+\frac{\mu}{2}(\mu-1)\right] .
\end{aligned}
$$

Next, substituting $p^{*}=0$ into (32) gives

$$
\begin{aligned}
\frac{q}{2}+\frac{r}{2}+\left[\frac{1}{2}+\right. & \left.\frac{\mu}{2}(\mu-1)\right]-q\left[\frac{1}{2}+\frac{\mu}{2}(\mu-1)\right]-r\left[\frac{1}{2}+\frac{\mu}{2}(\mu-1)\right] \\
& =\frac{q}{2}+\frac{r}{2}+\left[\frac{1}{2}+\frac{\mu}{2}(1-\mu)\right]-q\left[\frac{1}{2}+\frac{\mu}{2}(1-\mu)\right]-r\left[\frac{1}{2}+\frac{\mu}{2}(1-\mu)\right] .
\end{aligned}
$$

Finally, substituting $p^{*}=0$ into (33) leads to

$$
\begin{aligned}
\frac{q}{2}+\frac{r}{2}+\left[\frac{1}{2}+\frac{\mu}{2}(1-\mu)\right]-q & {\left[\frac{1}{2}+\frac{\mu}{2}(1-\mu)\right]-r\left[\frac{1}{2}+\frac{\mu}{2}(1-\mu)\right] } \\
= & q\left[\frac{1}{2}+\frac{\mu}{2}(1-\mu)\right]+r\left[\frac{1}{2}+\frac{\mu}{2}(\mu-1)\right]+\frac{1}{2}-\frac{q}{2}-\frac{r}{2} .
\end{aligned}
$$

By simplifying (39), taking common factors, and noting that

$$
r\left[1-\left[\frac{1}{2}+\frac{\mu}{2}(1-\mu)\right]-\left[\frac{1}{2}+\frac{\mu}{2}(\mu-1)\right]\right]=r\left[\frac{\mu}{2}(\mu-1)-\frac{\mu}{2}(\mu-1)\right]=0,
$$

I find that

$$
-q\left[1-2\left[\frac{1}{2}+\frac{\mu}{2}(\mu-1)\right]\right]+\frac{1}{2}-\left[\frac{1}{2}+\frac{\mu}{2}(\mu-1)\right]=0
$$

and thus

$$
q \mu(\mu-1)-\frac{\mu}{2}(\mu-1)=0 \Rightarrow q^{*}=\frac{\frac{\mu}{2}(\mu-1)}{\mu(\mu-1)} \Rightarrow q^{*}=\frac{1}{2} .
$$

Similarly, simplifying (41), taking common factors, and using (42) gives

$$
-q\left[1-2\left[\frac{1}{2}+\frac{\mu}{2}(1-\mu)\right]\right]+\frac{1}{2}-\left[\frac{1}{2}+\frac{\mu}{2}(1-\mu)\right]=0,
$$

which implies

$$
q \mu(1-\mu)-\frac{\mu}{2}(1-\mu)=0 \Rightarrow q^{*}=\frac{\frac{\mu}{2}(1-\mu)}{\mu(1-\mu)}=\frac{1}{2} .
$$

Finally, simplifying (40) obtains

$$
\begin{aligned}
{\left[\frac{1}{2}+\frac{\mu}{2}(\mu-1)\right]-q\left[\frac{1}{2}+\frac{\mu}{2}(\mu-1)\right]-r\left[\frac{1}{2}+\frac{\mu}{2}(\mu-1)\right] } \\
=\left[\frac{1}{2}+\frac{\mu}{2}(1-\mu)\right]-q\left[\frac{1}{2}+\frac{\mu}{2}(1-\mu)\right]-r\left[\frac{1}{2}+\frac{\mu}{2}(1-\mu)\right] .
\end{aligned}
$$


Since $\left[\frac{1}{2}+\frac{\mu}{2}(1-\mu)\right]>\left[\frac{1}{2}+\frac{\mu}{2}(\mu-1)\right]$ condition (45) is uniquely satisfied by $r=1-q$ as

$$
\begin{aligned}
{\left[\frac{1}{2}+\frac{\mu}{2}(\mu-1)\right]-q[} & {\left[\frac{1}{2}+\frac{\mu}{2}(\mu-1)\right]-(1-q)\left[\frac{1}{2}+\frac{\mu}{2}(\mu-1)\right] } \\
& =\left[\frac{1}{2}+\frac{\mu}{2}(1-\mu)\right]-q\left[\frac{1}{2}+\frac{\mu}{2}(1-\mu)\right]-(1-q)\left[\frac{1}{2}+\frac{\mu}{2}(\mu-1)\right]
\end{aligned}
$$

equals

$$
\begin{aligned}
& {\left[\frac{1}{2}+\frac{\mu}{2}(\mu-1)\right]-q\left[\frac{1}{2}+\frac{\mu}{2}(\mu-1)\right]-\left[\frac{1}{2}+\frac{\mu}{2}(\mu-1)\right]+q\left[\frac{1}{2}+\frac{\mu}{2}(\mu-1)\right]} \\
& =\left[\frac{1}{2}+\frac{\mu}{2}(1-\mu)\right]-q\left[\frac{1}{2}+\frac{\mu}{2}(1-\mu)\right]-\left[\frac{1}{2}+\frac{\mu}{2}(1-\mu)\right]+q\left[\frac{1}{2}+\frac{\mu}{2}(1-\mu)\right],
\end{aligned}
$$

which simplifies to 0 on both sides. This implies that $r^{*}=1-q^{*}=1-\frac{1}{2}=\frac{1}{2}$. Note again that equations (43), (44) and (46) are independent of $\operatorname{Pr}\left(\theta_{i}=\theta_{H}\right)$. Therefore, the vector $\left(p^{*}, q^{*}, r^{*}\right)=\left(0, \frac{1}{2}, \frac{1}{2}\right)$ makes player $j$ indifferent between her pure strategies for all $\operatorname{Pr}\left(\theta_{i}=\theta_{H}\right)$. As player $i$ and $j$ are symmetric, and were arbitrarily assigned, this vector constitutes a mixed strategy Bayes-Nash equilibrium.

This equilibrium is equivalent to each player $i \in \mathscr{I}$ using the strategy $\hat{\sigma}_{i}\left(\theta_{i}\right)=\mathbb{E}_{k}\left[Q\left(\theta_{i}, k\right)\right]$ as the mixed strategy determined by the vector $\left(p^{*}, q^{*}, r^{*}\right)=\left(0, \frac{1}{2}, \frac{1}{2}\right)$ is

$$
\begin{aligned}
\hat{\sigma}\left(\theta_{L}\right) & =q^{*} Q\left(\theta_{L}, k_{H}\right)+r^{*} Q\left(\theta_{L}, k_{L}\right), \\
& =\frac{1}{2}\left(\theta_{L}+k_{H}\right)+\frac{\theta_{L}}{2}=\theta_{L}+\frac{k_{H}}{2} .
\end{aligned}
$$

when player $i$ has low ability, $\theta_{i}=\theta_{L}$, and

$$
\begin{aligned}
\hat{\sigma}\left(\theta_{H}\right) & =q^{*} Q\left(\theta_{H}, k_{L}\right)+r^{*} Q\left(\theta_{H}, k_{H}\right), \\
& =\frac{\theta_{H}}{2}+\frac{1}{2}\left(\theta_{H}+k_{H}\right)=\theta_{H}+\frac{k_{H}}{2}
\end{aligned}
$$

when player $i$ has high ability, $\theta_{i}=\theta_{H}$. Hence, as (47) and (48) are equivalent to (37) for a given realisation of $\theta_{i}$, player $i$ 's mixed strategy $\hat{\sigma}_{i}\left(\theta_{i}\right)$ is equivalent to using a strategy based upon her expected performance $\mathbb{E}_{k}\left[Q\left(\theta_{i}, k\right)\right]$. This completes the proof of Proposition 4.

Proof of Theorem 1. By following the procedure laid out in Subsection 4.2 I can derive the players' expected payoffs $\mathbb{E}_{\theta, k}\left[u_{i}\left(\sigma, \theta_{i}\right)\right]$ when there is both uncertainty over the players' abilities and over the state of nature. Collating these expected payoffs for each possible strategy profile $\sigma=\left(\sigma_{1}\left(\theta_{1}\right), \sigma_{2}\left(\theta_{2}\right)\right)$ leads to the Bayesian strategic form of the game illustrated in Figure 11.

I first show that the pair of inequalities $1-2 \lambda>\mu$ and $\mu>2 \lambda$ imply that $\lambda<1 / 4$. Putting these two inequalities together yields $1-2 \lambda>\mu>2 \lambda$, which implies $1-2 \lambda>2 \lambda$. This inequality then implies that $1>4 \lambda$ and, hence, that $1 / 4>\lambda$.

I will now show that these conditions are sufficient for the existence of a pure strategy BayesNash equilibrium in which, for each $i \in \mathscr{I}$, the strategy $\sigma_{i}\left(\theta_{i}\right)=\left(Q\left(\theta_{H}, k_{H}\right) Q\left(\theta_{L}, k_{H}\right)\right)$ is a best 


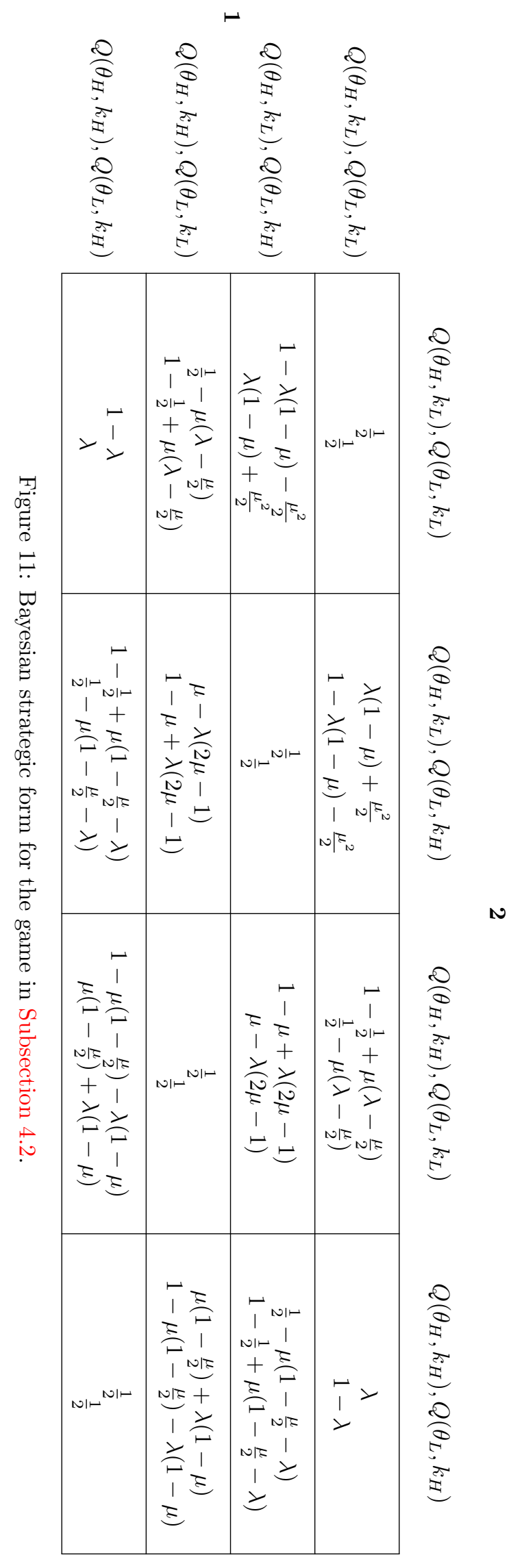


response to any strategy of player $j \in \mathscr{I} \backslash\{i\}$ as in the proof of Proposition 2. Suppose first that player $j$ uses the strategy $\sigma_{j}\left(\theta_{j}\right)=\left(Q\left(\theta_{H}, k_{L}\right) Q\left(\theta_{L}, k_{L}\right)\right)$, then, player $i$ 's strategy is a best response if

$$
\begin{aligned}
& \mathbb{E}_{\theta, k}\left[u_{i}\left(\left(Q\left(\theta_{H}, k_{H}\right) Q\left(\theta_{L}, k_{H}\right), Q\left(\theta_{H}, k_{L}\right) Q\left(\theta_{L}, k_{L}\right)\right), \theta_{i}\right)\right] \\
& >\mathbb{E}_{\theta, k}\left[u_{i}\left(\left(Q\left(\theta_{H}, k_{L}\right) Q\left(\theta_{L}, k_{L}\right), Q\left(\theta_{H}, k_{L}\right) Q\left(\theta_{L}, k_{L}\right)\right), \theta_{i}\right)\right] \\
& \Leftrightarrow 1-\lambda>\frac{1}{2} \Leftrightarrow \lambda<\frac{1}{2}, \\
& \mathbb{E}_{\theta, k}\left[u_{i}\left(\left(Q\left(\theta_{H}, k_{H}\right) Q\left(\theta_{L}, k_{H}\right), Q\left(\theta_{H}, k_{L}\right) Q\left(\theta_{L}, k_{L}\right)\right), \theta_{i}\right)\right] \\
& >\mathbb{E}_{\theta, k}\left[u_{i}\left(\left(Q\left(\theta_{H}, k_{L}\right) Q\left(\theta_{L}, k_{H}\right), Q\left(\theta_{H}, k_{L}\right) Q\left(\theta_{L}, k_{L}\right)\right), \theta_{i}\right)\right] \\
& \Leftrightarrow 1-\lambda>1-\lambda(1-\mu)-\frac{\mu^{2}}{2} \Leftrightarrow \mu>2 \lambda
\end{aligned}
$$

and

$$
\begin{aligned}
\mathbb{E}_{\theta, k}\left[u _ { i } \left(\left(Q\left(\theta_{H}, k_{H}\right)\right.\right.\right. & \left.\left.\left.Q\left(\theta_{L}, k_{H}\right), Q\left(\theta_{H}, k_{L}\right) Q\left(\theta_{L}, k_{L}\right)\right), \theta_{i}\right)\right] \\
> & \mathbb{E}_{\theta, k}\left[u_{i}\left(\left(Q\left(\theta_{H}, k_{H}\right) Q\left(\theta_{L}, k_{L}\right), Q\left(\theta_{H}, k_{L}\right) Q\left(\theta_{L}, k_{L}\right)\right), \theta_{i}\right)\right] \\
& \Leftrightarrow 1-\lambda>\frac{1}{2}-\mu\left(\lambda-\frac{\mu}{2}\right) \Leftrightarrow \frac{1}{2}\left(1-\mu^{2}\right)>\lambda(1-\mu) \Leftrightarrow \lambda<\frac{1}{2} .
\end{aligned}
$$

Condition (50) holds by the postulate of Theorem 1 that $1-2 \lambda>\mu>2 \lambda$. Conditions (49) and (51) hold by the implications of this postulate, as I showed above that these inequalities imply $\lambda<1 / 4$.

Suppose now that player $j$ uses the strategy $\sigma_{j}\left(\theta_{j}\right)=\left(Q\left(\theta_{H}, k_{L}\right) Q\left(\theta_{L}, k_{H}\right)\right)$. Player $i$ 's strategy is a best response to this if

$$
\begin{gathered}
\mathbb{E}_{\theta, k}\left[u_{i}\left(\left(Q\left(\theta_{H}, k_{H}\right) Q\left(\theta_{L}, k_{H}\right), Q\left(\theta_{H}, k_{L}\right) Q\left(\theta_{L}, k_{H}\right)\right), \theta_{i}\right)\right] \\
>\mathbb{E}_{\theta, k}\left[u_{i}\left(\left(Q\left(\theta_{H}, k_{L}\right) Q\left(\theta_{L}, k_{L}\right), Q\left(\theta_{H}, k_{L}\right) Q\left(\theta_{L}, k_{H}\right)\right), \theta_{i}\right)\right] \\
\Leftrightarrow 1-\frac{1}{2}+\mu\left(1-\frac{\mu}{2}-\lambda\right)>\lambda(1-\mu)+\frac{\mu^{2}}{2} \Leftrightarrow \lambda<\frac{1}{2}, \\
\mathbb{E}_{\theta, k}\left[u_{i}\left(\left(Q\left(\theta_{H}, k_{H}\right) Q\left(\theta_{L}, k_{H}\right), Q\left(\theta_{H}, k_{L}\right) Q\left(\theta_{L}, k_{H}\right)\right), \theta_{i}\right)\right] \\
>\mathbb{E}_{\theta, k}\left[u_{i}\left(\left(Q\left(\theta_{H}, k_{L}\right) Q\left(\theta_{L}, k_{H}\right), Q\left(\theta_{H}, k_{L}\right) Q\left(\theta_{L}, k_{H}\right)\right), \theta_{i}\right)\right] \\
\Leftrightarrow 1-\frac{1}{2}+\mu\left(1-\frac{\mu}{2}-\lambda\right)>\frac{1}{2} \Leftrightarrow \lambda<\frac{1}{2}
\end{gathered}
$$


and

$$
\begin{aligned}
& \mathbb{E}_{\theta, k}\left[u_{i}\left(\left(Q\left(\theta_{H}, k_{H}\right) Q\left(\theta_{L}, k_{H}\right), Q\left(\theta_{H}, k_{L}\right) Q\left(\theta_{L}, k_{H}\right)\right), \theta_{i}\right)\right] \\
& >\mathbb{E}_{\theta, k}\left[u_{i}\left(\left(Q\left(\theta_{H}, k_{H}\right) Q\left(\theta_{L}, k_{L}\right), Q\left(\theta_{H}, k_{L}\right) Q\left(\theta_{L}, k_{H}\right)\right), \theta_{i}\right)\right] \\
& \Leftrightarrow 1-\frac{1}{2}+\mu\left(1-\frac{\mu}{2}-\lambda\right)>\mu-\lambda(2 \mu-1) \Leftrightarrow \lambda<\frac{1}{2} .
\end{aligned}
$$

Conditions (52)-(54) are satisfied by the postulate of Theorem 1 that $1-2 \lambda>\mu>2 \lambda$.

Next, suppose that player $j$ uses the strategy $\sigma_{j}\left(\theta_{j}\right)=\left(Q\left(\theta_{H}, k_{H}\right) Q\left(\theta_{L}, k_{L}\right)\right)$. Player $i$ 's strategy is a best response to this if

$$
\begin{aligned}
& \mathbb{E}_{\theta, k}\left[u_{i}\left(\left(Q\left(\theta_{H}, k_{H}\right) Q\left(\theta_{L}, k_{H}\right), Q\left(\theta_{H}, k_{H}\right) Q\left(\theta_{L}, k_{L}\right)\right), \theta_{i}\right)\right] \\
& >\mathbb{E}_{\theta, k}\left[u_{i}\left(\left(Q\left(\theta_{H}, k_{L}\right) Q\left(\theta_{L}, k_{L}\right), Q\left(\theta_{H}, k_{H}\right) Q\left(\theta_{L}, k_{L}\right)\right), \theta_{i}\right)\right] \\
& \Leftrightarrow 1-\mu\left(1-\frac{\mu}{2}\right)-\lambda(1-\mu)>1-\frac{1}{2}+\mu\left(\lambda-\frac{\mu}{2}\right) \Leftrightarrow \lambda<\frac{1}{4},
\end{aligned}
$$

$$
\begin{aligned}
\mathbb{E}_{\theta, k}\left[u_{i}\left(\left(Q\left(\theta_{H}, k_{H}\right) Q\left(\theta_{L}, k_{H}\right), Q\left(\theta_{H}, k_{H}\right) Q\left(\theta_{L}, k_{L}\right)\right), \theta_{i}\right)\right] \\
\quad>\mathbb{E}_{\theta, k}\left[u_{i}\left(\left(Q\left(\theta_{H}, k_{L}\right) Q\left(\theta_{L}, k_{H}\right), Q\left(\theta_{H}, k_{H}\right) Q\left(\theta_{L}, k_{L}\right)\right), \theta_{i}\right)\right] \\
\Leftrightarrow 1-\mu\left(1-\frac{\mu}{2}\right)-\lambda(1-\mu)>1-\mu+\lambda(2 \mu-1) \Leftrightarrow \lambda<\frac{1}{2} \text { and } 2 \lambda<\mu
\end{aligned}
$$

and

$$
\begin{aligned}
& \mathbb{E}_{\theta, k}\left[u_{i}\left(\left(Q\left(\theta_{H}, k_{H}\right) Q\left(\theta_{L}, k_{H}\right), Q\left(\theta_{H}, k_{H}\right) Q\left(\theta_{L}, k_{L}\right)\right), \theta_{i}\right)\right] \\
&>\mathbb{E}_{\theta, k}\left[u_{i}\left(\left(Q\left(\theta_{H}, k_{H}\right) Q\left(\theta_{L}, k_{L}\right), Q\left(\theta_{H}, k_{H}\right) Q\left(\theta_{L}, k_{L}\right)\right), \theta_{i}\right)\right] \\
& \Leftrightarrow 1-\mu\left(1-\frac{\mu}{2}\right)-\lambda(1-\mu)>\frac{1}{2} \Leftrightarrow \lambda<\frac{1}{2} \text { and } \mu<1-2 \lambda .
\end{aligned}
$$

Conditions (55)-(57) are satisfied by the postulate of Theorem 1 that $1-2 \lambda>\mu>2 \lambda$.

Finally, suppose that player $j$ uses the strategy $\sigma_{j}\left(\theta_{j}\right)=\left(Q\left(\theta_{H}, k_{H}\right) Q\left(\theta_{L}, k_{H}\right)\right)$. Player $i$ 's strategy is a best response to this if

$$
\begin{aligned}
& \mathbb{E}_{\theta, k}\left[u_{i}\left(\left(Q\left(\theta_{H}, k_{H}\right) Q\left(\theta_{L}, k_{H}\right), Q\left(\theta_{H}, k_{H}\right) Q\left(\theta_{L}, k_{H}\right)\right), \theta_{i}\right)\right] \\
& >\mathbb{E}_{\theta, k}\left[u_{i}\left(\left(Q\left(\theta_{H}, k_{L}\right) Q\left(\theta_{L}, k_{L}\right), Q\left(\theta_{H}, k_{H}\right) Q\left(\theta_{L}, k_{H}\right)\right), \theta_{i}\right)\right] \Leftrightarrow \frac{1}{2}>\lambda, \\
& \mathbb{E}_{\theta, k}\left[u_{i}\left(\left(Q\left(\theta_{H}, k_{H}\right) Q\left(\theta_{L}, k_{H}\right), Q\left(\theta_{H}, k_{H}\right) Q\left(\theta_{L}, k_{H}\right)\right), \theta_{i}\right)\right] \\
& >\mathbb{E}_{\theta, k}\left[u_{i}\left(\left(Q\left(\theta_{H}, k_{L}\right) Q\left(\theta_{L}, k_{H}\right), Q\left(\theta_{H}, k_{H}\right) Q\left(\theta_{L}, k_{H}\right)\right), \theta_{i}\right)\right] \\
& \Leftrightarrow \frac{1}{2}>\frac{1}{2}-\mu\left(1-\frac{\mu}{2}-\lambda\right) \Leftrightarrow \lambda<\frac{1}{2}
\end{aligned}
$$


and

$$
\begin{aligned}
\mathbb{E}_{\theta, k}\left[u_{i}\left(\left(Q\left(\theta_{H}, k_{H}\right) Q\left(\theta_{L}, k_{H}\right), Q\left(\theta_{H}, k_{H}\right) Q\left(\theta_{L}, k_{H}\right)\right), \theta_{i}\right)\right] \\
>\mathbb{E}_{\theta, k}\left[u_{i}\left(\left(Q\left(\theta_{H}, k_{H}\right) Q\left(\theta_{L}, k_{L}\right), Q\left(\theta_{H}, k_{H}\right) Q\left(\theta_{L}, k_{H}\right)\right), \theta_{i}\right)\right] \\
\Leftrightarrow \frac{1}{2}>\mu\left(1-\frac{\mu}{2}\right)+\lambda(1-\mu) \Leftrightarrow \lambda<\frac{1}{2} \text { and } \mu<1-2 \lambda .
\end{aligned}
$$

As before, Conditions (58)-(60) are satisfied by the postulate of Theorem 1 that $1-2 \lambda>\mu>2 \lambda$.

Therefore, I have shown that, irrespective of player $j$ 's strategy, player $i$ 's best response is $\sigma_{i}\left(\theta_{i}\right)=\left(Q\left(\theta_{H}, k_{H}\right) Q\left(\theta_{L}, k_{H}\right)\right)$. As $i, j \in \mathscr{I}$ were assigned arbitrarily and the players' payoffs are symmetric this implies that the strategy profile $\sigma^{*}=\left(Q\left(\theta_{H}, k_{H}\right) Q\left(\theta_{L}, k_{H}\right), Q\left(\theta_{H}, k_{H}\right) Q\left(\theta_{L}, k_{H}\right)\right)$ is the unique pure strategy Bayes-Nash equilibrium of the game in Figure 11. I have also demonstrated that the postulate of Theorem 1 that $1-2 \lambda>\mu>2 \lambda$ is a sufficient condition for the existence of this equilibrium.

I next show that the pair of inequalities $2 \lambda-1>\mu$ and $\mu>2(1-\lambda)$ imply that $\lambda>3 / 4$. Putting these two inequalities together yields $2 \lambda-1>\mu>2(1-\lambda)$, which implies $2 \lambda-1>$ $2(1-\lambda)$. This inequality then implies that $4 \lambda>3$ and, hence, that $3 / 4<\lambda$.

I will now show that these conditions are sufficient for the existence of a pure strategy BayesNash equilibrium in which, for each $i \in \mathscr{I}$, the strategy $\sigma_{i}\left(\theta_{i}\right)=\left(Q\left(\theta_{H}, k_{L}\right) Q\left(\theta_{L}, k_{L}\right)\right)$ is a best response to any strategy of player $j \in \mathscr{I} \backslash\{i\}$. Suppose first that player $j$ uses the strategy $\sigma_{j}\left(\theta_{j}\right)=\left(Q\left(\theta_{H}, k_{L}\right) Q\left(\theta_{L}, k_{L}\right)\right)$, then, player $i$ 's strategy is a best response if

$$
\begin{aligned}
& \mathbb{E}_{\theta, k}\left[u_{i}\left(\left(Q\left(\theta_{H}, k_{L}\right) Q\left(\theta_{L}, k_{L}\right), Q\left(\theta_{H}, k_{L}\right) Q\left(\theta_{L}, k_{L}\right)\right), \theta_{i}\right)\right] \\
& >\mathbb{E}_{\theta, k}\left[u_{i}\left(\left(Q\left(\theta_{H}, k_{L}\right) Q\left(\theta_{L}, k_{H}\right), Q\left(\theta_{H}, k_{L}\right) Q\left(\theta_{L}, k_{L}\right)\right), \theta_{i}\right)\right] \\
& \Leftrightarrow \frac{1}{2}>1-\lambda(1-\mu)-\frac{\mu^{2}}{2} \Leftrightarrow \lambda>\frac{1}{2} \text { and } 2 \lambda-1>\mu,
\end{aligned}
$$

$$
\begin{gathered}
\mathbb{E}_{\theta, k}\left[u_{i}\left(\left(Q\left(\theta_{H}, k_{L}\right) Q\left(\theta_{L}, k_{L}\right), Q\left(\theta_{H}, k_{L}\right) Q\left(\theta_{L}, k_{L}\right)\right), \theta_{i}\right)\right] \\
>\mathbb{E}_{\theta, k}\left[u_{i}\left(\left(Q\left(\theta_{H}, k_{H}\right) Q\left(\theta_{L}, k_{L}\right), Q\left(\theta_{H}, k_{L}\right) Q\left(\theta_{L}, k_{L}\right)\right), \theta_{i}\right)\right] \\
\Leftrightarrow \frac{1}{2}>\frac{1}{2}-\mu\left(\lambda-\frac{\mu}{2}\right) \Leftrightarrow \lambda>\frac{1}{2}
\end{gathered}
$$

and

$$
\begin{aligned}
& \mathbb{E}_{\theta, k}\left[u_{i}\left(\left(Q\left(\theta_{H}, k_{L}\right) Q\left(\theta_{L}, k_{L}\right), Q\left(\theta_{H}, k_{L}\right) Q\left(\theta_{L}, k_{L}\right)\right), \theta_{i}\right)\right] \\
&>\mathbb{E}_{\theta, k}\left[u_{i}\left(\left(Q\left(\theta_{H}, k_{H}\right) Q\left(\theta_{L}, k_{H}\right), Q\left(\theta_{H}, k_{L}\right) Q\left(\theta_{L}, k_{L}\right)\right), \theta_{i}\right)\right] \\
& \Leftrightarrow \frac{1}{2}>1-\lambda \Leftrightarrow \lambda>\frac{1}{2} .
\end{aligned}
$$

The second condition in (61) holds by the postulate of Theorem 1 that $2 \lambda-1>\mu>2(1-\lambda)$. The first condition in (61) and conditions (62) and (63) hold by the implications of this postulate, 
as I showed above that these inequalities imply $\lambda>3 / 4$.

Suppose now that player $j$ uses the strategy $\sigma_{j}\left(\theta_{j}\right)=\left(Q\left(\theta_{H}, k_{L}\right) Q\left(\theta_{L}, k_{H}\right)\right)$. Player $i$ 's strategy is a best response to this if

$$
\begin{aligned}
& \mathbb{E}_{\theta, k}\left[u_{i}\left(\left(Q\left(\theta_{H}, k_{L}\right) Q\left(\theta_{L}, k_{L}\right), Q\left(\theta_{H}, k_{L}\right) Q\left(\theta_{L}, k_{H}\right)\right), \theta_{i}\right)\right] \\
& >\mathbb{E}_{\theta, k}\left[u_{i}\left(\left(Q\left(\theta_{H}, k_{L}\right) Q\left(\theta_{L}, k_{H}\right), Q\left(\theta_{H}, k_{L}\right) Q\left(\theta_{L}, k_{H}\right)\right), \theta_{i}\right)\right] \\
& \Leftrightarrow \lambda(1-\mu)+\frac{\mu^{2}}{2}>\frac{1}{2} \Leftrightarrow \lambda>\frac{1}{2} \text { and } 2 \lambda-1>\mu, \\
& \mathbb{E}_{\theta, k}\left[u_{i}\left(\left(Q\left(\theta_{H}, k_{L}\right) Q\left(\theta_{L}, k_{L}\right), Q\left(\theta_{H}, k_{L}\right) Q\left(\theta_{L}, k_{H}\right)\right), \theta_{i}\right)\right] \\
& >\mathbb{E}_{\theta, k}\left[u_{i}\left(\left(Q\left(\theta_{H}, k_{H}\right) Q\left(\theta_{L}, k_{L}\right), Q\left(\theta_{H}, k_{L}\right) Q\left(\theta_{L}, k_{H}\right)\right), \theta_{i}\right)\right] \\
& \Leftrightarrow \lambda(1-\mu)+\frac{\mu^{2}}{2}>\mu-\lambda(2 \mu-1) \Leftrightarrow \lambda>\frac{1}{2} \text { and } \mu>2(1-\lambda)
\end{aligned}
$$

and

$$
\begin{aligned}
& \mathbb{E}_{\theta, k}\left[u_{i}\left(\left(Q\left(\theta_{H}, k_{L}\right) Q\left(\theta_{L}, k_{L}\right), Q\left(\theta_{H}, k_{L}\right) Q\left(\theta_{L}, k_{H}\right)\right), \theta_{i}\right)\right] \\
& >\mathbb{E}_{\theta, k}\left[u_{i}\left(\left(Q\left(\theta_{H}, k_{H}\right) Q\left(\theta_{L}, k_{H}\right), Q\left(\theta_{H}, k_{L}\right) Q\left(\theta_{L}, k_{H}\right)\right), \theta_{i}\right)\right] \\
& \Leftrightarrow \lambda(1-\mu)+\frac{\mu^{2}}{2}>1-\frac{1}{2}+\mu\left(1-\frac{\mu}{2}-\lambda\right) \Leftrightarrow \lambda>\frac{3}{4} .
\end{aligned}
$$

Conditions (64)-(66) are satisfied by the postulate of Theorem 1 that $2 \lambda-1>\mu>2(1-\lambda)$.

Next, suppose that player $j$ uses the strategy $\sigma_{j}\left(\theta_{j}\right)=\left(Q\left(\theta_{H}, k_{H}\right) Q\left(\theta_{L}, k_{L}\right)\right)$. Player $i$ 's strategy is a best response to this if

$$
\begin{aligned}
& \mathbb{E}_{\theta, k}\left[u_{i}\left(\left(Q\left(\theta_{H}, k_{L}\right) Q\left(\theta_{L}, k_{L}\right), Q\left(\theta_{H}, k_{H}\right) Q\left(\theta_{L}, k_{L}\right)\right), \theta_{i}\right)\right] \\
& >\mathbb{E}_{\theta, k}\left[u_{i}\left(\left(Q\left(\theta_{H}, k_{L}\right) Q\left(\theta_{L}, k_{H}\right), Q\left(\theta_{H}, k_{H}\right) Q\left(\theta_{L}, k_{L}\right)\right), \theta_{i}\right)\right] \\
& \Leftrightarrow 1-\frac{1}{2}+\mu\left(\lambda-\frac{\mu}{2}\right)>1-\mu+\lambda(2 \mu-1) \Leftrightarrow \lambda>\frac{1}{2}, \\
& \mathbb{E}_{\theta, k}\left[u_{i}\left(\left(Q\left(\theta_{H}, k_{L}\right) Q\left(\theta_{L}, k_{L}\right), Q\left(\theta_{H}, k_{H}\right) Q\left(\theta_{L}, k_{L}\right)\right), \theta_{i}\right)\right] \\
& >\mathbb{E}_{\theta, k}\left[u_{i}\left(\left(Q\left(\theta_{H}, k_{H}\right) Q\left(\theta_{L}, k_{L}\right), Q\left(\theta_{H}, k_{H}\right) Q\left(\theta_{L}, k_{L}\right)\right), \theta_{i}\right)\right] \\
& \Leftrightarrow 1-\frac{1}{2}+\mu\left(\lambda-\frac{\mu}{2}\right)>\frac{1}{2} \Leftrightarrow \lambda>\frac{1}{2}
\end{aligned}
$$


and

$$
\begin{aligned}
& \mathbb{E}_{\theta, k}\left[u_{i}\left(\left(Q\left(\theta_{H}, k_{L}\right) Q\left(\theta_{L}, k_{L}\right), Q\left(\theta_{H}, k_{H}\right) Q\left(\theta_{L}, k_{L}\right)\right), \theta_{i}\right)\right] \\
& >\mathbb{E}_{\theta, k}\left[u_{i}\left(\left(Q\left(\theta_{H}, k_{H}\right) Q\left(\theta_{L}, k_{H}\right), Q\left(\theta_{H}, k_{H}\right) Q\left(\theta_{L}, k_{L}\right)\right), \theta_{i}\right)\right] \\
& \Leftrightarrow 1-\frac{1}{2}+\mu\left(\lambda-\frac{\mu}{2}\right)>1-\mu\left(1-\frac{\mu}{2}\right)-\lambda(1-\mu) \Leftrightarrow \lambda>\frac{1}{2} .
\end{aligned}
$$

Conditions (67)-(69) are satisfied by the implications of the postulate of Theorem 1 that $2 \lambda-1>$ $\mu>2(1-\lambda)$.

Finally, suppose that player $j$ uses the strategy $\sigma_{j}\left(\theta_{j}\right)=\left(Q\left(\theta_{H}, k_{H}\right) Q\left(\theta_{L}, k_{H}\right)\right)$. Player $i$ 's strategy is a best response to this if

$$
\begin{aligned}
& \mathbb{E}_{\theta, k}\left[u_{i}\left(\left(Q\left(\theta_{H}, k_{L}\right) Q\left(\theta_{L}, k_{L}\right), Q\left(\theta_{H}, k_{H}\right) Q\left(\theta_{L}, k_{H}\right)\right), \theta_{i}\right)\right] \\
&>\mathbb{E}_{\theta, k}\left[u_{i}\left(\left(Q\left(\theta_{H}, k_{L}\right) Q\left(\theta_{L}, k_{H}\right), Q\left(\theta_{H}, k_{H}\right) Q\left(\theta_{L}, k_{H}\right)\right), \theta_{i}\right)\right] \\
& \Leftrightarrow \lambda>\frac{1}{2}-\mu\left(1-\frac{\mu}{2}-\lambda\right) \Leftrightarrow \lambda>\frac{1}{2}
\end{aligned}
$$

and

$$
\begin{aligned}
& \mathbb{E}_{\theta, k}\left[u_{i}\left(\left(Q\left(\theta_{H}, k_{L}\right) Q\left(\theta_{L}, k_{L}\right), Q\left(\theta_{H}, k_{H}\right) Q\left(\theta_{L}, k_{H}\right)\right), \theta_{i}\right)\right] \\
& >\mathbb{E}_{\theta, k}\left[u_{i}\left(\left(Q\left(\theta_{H}, k_{H}\right) Q\left(\theta_{L}, k_{L}\right), Q\left(\theta_{H}, k_{H}\right) Q\left(\theta_{L}, k_{H}\right)\right), \theta_{i}\right)\right] \\
& \Leftrightarrow \lambda>\mu\left(1-\frac{\mu}{2}\right)+\lambda(1-\mu) \Leftrightarrow \lambda>\frac{1}{2} \text { and } \mu>2(1-\lambda) .
\end{aligned}
$$

Conditions (70) and (71) are satisfied by the postulate of Theorem 1 that $2 \lambda-1>\mu>2(1-\lambda)$.

Therefore, I have shown that, irrespective of player $j$ 's strategy, player $i$ 's best response is $\sigma_{i}\left(\theta_{i}\right)=\left(Q\left(\theta_{H}, k_{L}\right) Q\left(\theta_{L}, k_{L}\right)\right)$. As $i, j \in \mathscr{I}$ were assigned arbitrarily and the players' payoffs are symmetric this implies that the strategy profile $\sigma^{*}=\left(Q\left(\theta_{H}, k_{L}\right) Q\left(\theta_{L}, k_{L}\right), Q\left(\theta_{H}, k_{L}\right) Q\left(\theta_{L}, k_{L}\right)\right)$ is the unique pure strategy Bayes-Nash equilibrium of the game in Figure 11. I have also demonstrated that the postulate of Theorem 1 that $2 \lambda-1>\mu>2(1-\lambda)$ is a sufficient condition for the existence of this equilibrium. This completes the proof of Theorem 1.

Proof of Theorem 2. Relaxing Assumption 2 means that the players' expected payoffs $\mathbb{E}_{\theta, k}\left[u_{i}\left(\sigma, \theta_{i}\right)\right]$ change relative to those in Figure 11. The resulting expected payoffs are expressed in Figure 12. In contrast to Theorem 1, I first show that $1 / 2>\lambda>0$ is sufficient for the existence of a pure strategy Bayes-Nash equilibrium in which, for each $i \in \mathscr{I}$, the strategy $\sigma_{i}\left(\theta_{i}\right)=\left(Q\left(\theta_{H}, k_{H}\right) Q\left(\theta_{L}, k_{H}\right)\right)$ is a best response to any strategy of player $j \in \mathscr{I} \backslash\{i\}$. Note that this condition is equivalent to $\operatorname{Pr}\left(k=k_{H}\right)>\operatorname{Pr}\left(k=k_{L}\right)$. Suppose first that player $j$ uses 


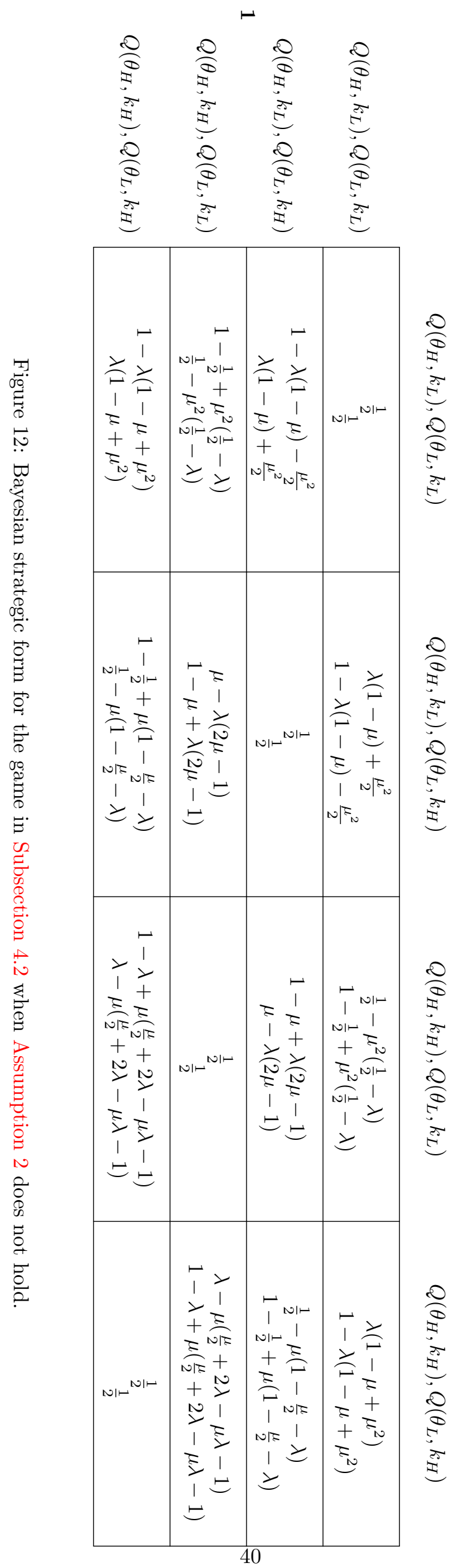


the strategy $\sigma_{j}\left(\theta_{j}\right)=\left(Q\left(\theta_{H}, k_{L}\right) Q\left(\theta_{L}, k_{L}\right)\right)$, then, player $i$ 's strategy is a best response if

$$
\begin{aligned}
& \mathbb{E}_{\theta, k}\left[u_{i}\left(\left(Q\left(\theta_{H}, k_{H}\right) Q\left(\theta_{L}, k_{H}\right), Q\left(\theta_{H}, k_{L}\right) Q\left(\theta_{L}, k_{L}\right)\right), \theta_{i}\right)\right] \\
& >\mathbb{E}_{\theta, k}\left[u_{i}\left(\left(Q\left(\theta_{H}, k_{L}\right) Q\left(\theta_{L}, k_{L}\right), Q\left(\theta_{H}, k_{L}\right) Q\left(\theta_{L}, k_{L}\right)\right), \theta_{i}\right)\right] \\
& \Leftrightarrow 1-\lambda(1-\mu(1-\mu))>\frac{1}{2} \Leftrightarrow \frac{1}{2}>\lambda(1-\mu(1-\mu)) \Leftrightarrow \frac{1}{2}>\lambda
\end{aligned}
$$

$$
\begin{aligned}
& \mathbb{E}_{\theta, k}\left[u_{i}\left(\left(Q\left(\theta_{H}, k_{H}\right) Q\left(\theta_{L}, k_{H}\right), Q\left(\theta_{H}, k_{L}\right) Q\left(\theta_{L}, k_{L}\right)\right), \theta_{i}\right)\right] \\
&> \mathbb{E}_{\theta, k}\left[u_{i}\left(\left(Q\left(\theta_{H}, k_{L}\right) Q\left(\theta_{L}, k_{H}\right), Q\left(\theta_{H}, k_{L}\right) Q\left(\theta_{L}, k_{L}\right)\right), \theta_{i}\right)\right] \\
& \Leftrightarrow 1-\lambda(1-\mu(1-\mu))>1-\lambda(1-\mu)-\frac{\mu^{2}}{2} \Leftrightarrow \frac{\mu^{2}}{2}>\lambda \mu^{2} \Leftrightarrow \frac{1}{2}>\lambda
\end{aligned}
$$

and

$$
\begin{aligned}
& \mathbb{E}_{\theta, k}\left[u_{i}\left(\left(Q\left(\theta_{H}, k_{H}\right) Q\left(\theta_{L}, k_{H}\right), Q\left(\theta_{H}, k_{L}\right) Q\left(\theta_{L}, k_{L}\right)\right), \theta_{i}\right)\right] \\
& \quad>\mathbb{E}_{\theta, k}\left[u_{i}\left(\left(Q\left(\theta_{H}, k_{H}\right) Q\left(\theta_{L}, k_{L}\right), Q\left(\theta_{H}, k_{L}\right) Q\left(\theta_{L}, k_{L}\right)\right), \theta_{i}\right)\right] \\
& \Leftrightarrow 1-\lambda(1-\mu(1-\mu))>1-\frac{1}{2}+\mu^{2}\left(\frac{1}{2}-\lambda\right) \Leftrightarrow \frac{1}{2}\left(1-\mu^{2}\right)>\lambda\left(1-\mu^{2}\right) \Rightarrow \frac{1}{2}>\lambda .
\end{aligned}
$$

Conditions (72)-(74) are satisfied by the postulate of Theorem 2 that $\frac{1}{2}>\lambda$.

Suppose now that player $j$ uses the strategy $\sigma_{j}\left(\theta_{j}\right)=\left(Q\left(\theta_{H}, k_{L}\right) Q\left(\theta_{L}, k_{H}\right)\right)$. The conditions required for player $i$ 's strategy to be a best response are unchanged from the proof of Theorem 1 and are given by (52)-(54). These conditions require that $\frac{1}{2}>\lambda$, which holds by the postulate of Theorem 2.

Next, suppose now that player $j$ uses the strategy $\sigma_{j}\left(\theta_{j}\right)=\left(Q\left(\theta_{H}, k_{H}\right) Q\left(\theta_{L}, k_{L}\right)\right)$. Player $i$ 's strategy is a best response to this if

$$
\begin{aligned}
\mathbb{E}_{\theta, k}\left[u_{i}\left(\left(Q\left(\theta_{H}, k_{H}\right) Q\left(\theta_{L}, k_{H}\right), Q\left(\theta_{H}, k_{H}\right) Q\left(\theta_{L}, k_{L}\right)\right), \theta_{i}\right)\right] \\
>\mathbb{E}_{\theta, k}\left[u_{i}\left(\left(Q\left(\theta_{H}, k_{L}\right) Q\left(\theta_{L}, k_{L}\right), Q\left(\theta_{H}, k_{H}\right) Q\left(\theta_{L}, k_{L}\right)\right), \theta_{i}\right)\right] \\
\Leftrightarrow \frac{1}{2}-\lambda+\mu\left(\frac{\mu}{2}+2 \lambda-\mu \lambda-1\right)>-\mu^{2}\left(\frac{1}{2}-\lambda\right) \Leftrightarrow \frac{1}{2}>\lambda,
\end{aligned}
$$

$$
\begin{aligned}
& \mathbb{E}_{\theta, k}\left[u_{i}\left(\left(Q\left(\theta_{H}, k_{H}\right) Q\left(\theta_{L}, k_{H}\right), Q\left(\theta_{H}, k_{H}\right) Q\left(\theta_{L}, k_{L}\right)\right), \theta_{i}\right)\right] \\
& \quad>\mathbb{E}_{\theta, k}\left[u_{i}\left(\left(Q\left(\theta_{H}, k_{L}\right) Q\left(\theta_{L}, k_{H}\right), Q\left(\theta_{H}, k_{H}\right) Q\left(\theta_{L}, k_{L}\right)\right), \theta_{i}\right)\right] \\
& \Leftrightarrow 1-\lambda+\mu\left(\frac{\mu}{2}+2 \lambda-\mu \lambda-1\right)>1-\mu+\lambda(2 \mu-1) \Leftrightarrow \frac{\mu^{2}}{2}>\lambda \mu^{2} \Leftrightarrow \frac{1}{2}>\lambda
\end{aligned}
$$


and

$$
\begin{aligned}
& \mathbb{E}_{\theta, k}\left[u_{i}\left(\left(Q\left(\theta_{H}, k_{H}\right) Q\left(\theta_{L}, k_{H}\right), Q\left(\theta_{H}, k_{H}\right) Q\left(\theta_{L}, k_{L}\right)\right), \theta_{i}\right)\right] \\
& >\mathbb{E}_{\theta, k}\left[u_{i}\left(\left(Q\left(\theta_{H}, k_{H}\right) Q\left(\theta_{L}, k_{L}\right), Q\left(\theta_{H}, k_{H}\right) Q\left(\theta_{L}, k_{L}\right)\right), \theta_{i}\right)\right] \\
& \Leftrightarrow 1-\lambda+\mu\left(\frac{\mu}{2}+2 \lambda-\mu \lambda-1\right)>\frac{1}{2} \Leftrightarrow \frac{1}{2}+\mu\left(\frac{\mu}{2}+2 \lambda-\mu \lambda-1\right)>\lambda \Leftrightarrow \frac{1}{2}>\lambda .
\end{aligned}
$$

Conditions (75)-(77) are satisfied by the postulate of Theorem 2 that $\frac{1}{2}>\lambda$.

Finally, suppose now that player $j$ uses the strategy $\sigma_{j}\left(\theta_{j}\right)=\left(Q\left(\theta_{H}, k_{H}\right) Q\left(\theta_{L}, k_{H}\right)\right)$. Player $i$ 's strategy is a best response to this if

$$
\begin{aligned}
& \mathbb{E}_{\theta, k}\left[u_{i}\left(\left(Q\left(\theta_{H}, k_{H}\right) Q\left(\theta_{L}, k_{H}\right), Q\left(\theta_{H}, k_{H}\right) Q\left(\theta_{L}, k_{H}\right)\right), \theta_{i}\right)\right] \\
&>\mathbb{E}_{\theta, k}\left[u_{i}\left(\left(Q\left(\theta_{H}, k_{L}\right) Q\left(\theta_{L}, k_{L}\right), Q\left(\theta_{H}, k_{H}\right) Q\left(\theta_{L}, k_{H}\right)\right), \theta_{i}\right)\right] \\
& \Leftrightarrow \frac{1}{2}>\lambda(1-\mu(1-\mu)) \Leftrightarrow \frac{1}{2}>\lambda,
\end{aligned}
$$

$$
\begin{aligned}
\mathbb{E}_{\theta, k}\left[u_{i}\left(\left(Q\left(\theta_{H}, k_{H}\right) Q\left(\theta_{L}, k_{H}\right), Q\left(\theta_{H}, k_{H}\right) Q\left(\theta_{L}, k_{H}\right)\right), \theta_{i}\right)\right] & \\
>\mathbb{E}_{\theta, k}\left[u_{i}\left(\left(Q\left(\theta_{H}, k_{L}\right) Q\left(\theta_{L}, k_{H}\right), Q\left(\theta_{H}, k_{H}\right) Q\left(\theta_{L}, k_{H}\right)\right), \theta_{i}\right)\right] & \Leftrightarrow \frac{1}{2}>\frac{1}{2}-\mu\left(1-\frac{\mu}{2}-\lambda\right) \Leftrightarrow \frac{1}{2}>\lambda
\end{aligned}
$$

and

$$
\begin{aligned}
\mathbb{E}_{\theta, k}\left[u_{i}\left(\left(Q\left(\theta_{H}, k_{H}\right) Q\left(\theta_{L}, k_{H}\right), Q\left(\theta_{H}, k_{H}\right) Q\left(\theta_{L}, k_{H}\right)\right), \theta_{i}\right)\right] & \\
>\mathbb{E}_{\theta, k}\left[u_{i}\left(\left(Q\left(\theta_{H}, k_{H}\right) Q\left(\theta_{L}, k_{L}\right), Q\left(\theta_{H}, k_{H}\right) Q\left(\theta_{L}, k_{H}\right)\right), \theta_{i}\right)\right] & \Leftrightarrow \frac{1}{2}>\lambda-\mu\left(\frac{\mu}{2}+2 \lambda-\mu \lambda-1\right) \Leftrightarrow \frac{1}{2}>\lambda .
\end{aligned}
$$

Conditions (78)-(80) are satisfied by the postulate of Theorem 2 that $\frac{1}{2}>\lambda$.

Therefore, I have shown that, irrespective of player $j$ 's strategy, player $i$ 's best response is $\sigma_{i}\left(\theta_{i}\right)=\left(Q\left(\theta_{H}, k_{H}\right) Q\left(\theta_{L}, k_{H}\right)\right)$. As $i, j \in \mathscr{I}$ were assigned arbitrarily and the players' payoffs are symmetric this implies that the strategy profile $\sigma^{*}=\left(Q\left(\theta_{H}, k_{H}\right) Q\left(\theta_{L}, k_{H}\right), Q\left(\theta_{H}, k_{H}\right) Q\left(\theta_{L}, k_{H}\right)\right)$ is the unique pure strategy Bayes-Nash equilibrium of the game in Figure 12 when $\operatorname{Pr}\left(k=k_{H}\right)>$ $\operatorname{Pr}\left(k=k_{L}\right)$.

I will now show, as in Theorem 1 , that $2 \lambda-1>\mu>2(1-\lambda)$ is sufficient for the existence of a pure strategy Bayes-Nash equilibrium in which, for each $i \in \mathscr{I}$, the strategy $\sigma_{i}\left(\theta_{i}\right)=$ $\left(Q\left(\theta_{H}, k_{L}\right) Q\left(\theta_{L}, k_{L}\right)\right)$ is a best response to any strategy of player $j \in \mathscr{I} \backslash\{i\}$. Suppose first that player $j$ uses the strategy $\sigma_{j}\left(\theta_{j}\right)=\left(Q\left(\theta_{H}, k_{L}\right) Q\left(\theta_{L}, k_{L}\right)\right)$, then, player $i$ 's strategy is a best 
response if

$$
\begin{aligned}
& \mathbb{E}_{\theta, k}\left[u_{i}\left(\left(Q\left(\theta_{H}, k_{L}\right) Q\left(\theta_{L}, k_{L}\right), Q\left(\theta_{H}, k_{L}\right) Q\left(\theta_{L}, k_{L}\right)\right), \theta_{i}\right)\right] \\
& >\mathbb{E}_{\theta, k}\left[u_{i}\left(\left(Q\left(\theta_{H}, k_{L}\right) Q\left(\theta_{L}, k_{H}\right), Q\left(\theta_{H}, k_{L}\right) Q\left(\theta_{L}, k_{L}\right)\right), \theta_{i}\right)\right] \\
& \Leftrightarrow \frac{1}{2}>1-\lambda(1-\mu)-\frac{\mu^{2}}{2} \Leftrightarrow \lambda>\frac{1}{2} \text { and } 2 \lambda-1>\mu,
\end{aligned}
$$

$$
\begin{gathered}
\mathbb{E}_{\theta, k}\left[u_{i}\left(\left(Q\left(\theta_{H}, k_{L}\right) Q\left(\theta_{L}, k_{L}\right), Q\left(\theta_{H}, k_{L}\right) Q\left(\theta_{L}, k_{L}\right)\right), \theta_{i}\right)\right] \\
>\mathbb{E}_{\theta, k}\left[u_{i}\left(\left(Q\left(\theta_{H}, k_{H}\right) Q\left(\theta_{L}, k_{L}\right), Q\left(\theta_{H}, k_{L}\right) Q\left(\theta_{L}, k_{L}\right)\right), \theta_{i}\right)\right] \\
\Leftrightarrow \frac{1}{2}>1-\frac{1}{2}+\mu^{2}\left(\frac{1}{2}-\lambda\right) \Leftrightarrow 0>\mu^{2}\left(\frac{1}{2}-\lambda\right) \Leftrightarrow \lambda>\frac{1}{2}
\end{gathered}
$$

and

$$
\begin{gathered}
\mathbb{E}_{\theta, k}\left[u_{i}\left(\left(Q\left(\theta_{H}, k_{L}\right) Q\left(\theta_{L}, k_{L}\right), Q\left(\theta_{H}, k_{L}\right) Q\left(\theta_{L}, k_{L}\right)\right), \theta_{i}\right)\right] \\
>\mathbb{E}_{\theta, k}\left[u_{i}\left(\left(Q\left(\theta_{H}, k_{H}\right) Q\left(\theta_{L}, k_{H}\right), Q\left(\theta_{H}, k_{L}\right) Q\left(\theta_{L}, k_{L}\right)\right), \theta_{i}\right)\right] \\
\Leftrightarrow \frac{1}{2}>1-\lambda(1-\mu(1-\mu)) \Leftrightarrow \lambda(1-\mu(1-\mu))>\frac{1}{2} \Leftrightarrow \lambda>\frac{2}{3} .
\end{gathered}
$$

Conditions (81)-(83) are satisfied by the postulate that $2 \lambda-1>\mu>2(1-\lambda)$ as I have shown that this implies that $\lambda>\frac{3}{4}$.

Suppose now that player $j$ uses the strategy $\sigma_{j}\left(\theta_{j}\right)=\left(Q\left(\theta_{H}, k_{L}\right) Q\left(\theta_{L}, k_{H}\right)\right)$. The conditions required for player $i$ 's strategy to be a best response are unchanged from the proof of Theorem 1 and are given by (64)-(66). These conditions require that $2 \lambda-1>\mu>2(1-\lambda)$, which holds by the postulate of Theorem 2 .

Next, suppose now that player $j$ uses the strategy $\sigma_{j}\left(\theta_{j}\right)=\left(Q\left(\theta_{H}, k_{H}\right) Q\left(\theta_{L}, k_{L}\right)\right)$. Player $i$ 's strategy is a best response to this if

$$
\begin{aligned}
& \mathbb{E}_{\theta, k}\left[u_{i}\left(\left(Q\left(\theta_{H}, k_{L}\right) Q\left(\theta_{L}, k_{L}\right), Q\left(\theta_{H}, k_{H}\right) Q\left(\theta_{L}, k_{L}\right)\right), \theta_{i}\right)\right] \\
& \quad>\mathbb{E}_{\theta, k}\left[u_{i}\left(\left(Q\left(\theta_{H}, k_{L}\right) Q\left(\theta_{L}, k_{H}\right), Q\left(\theta_{H}, k_{H}\right) Q\left(\theta_{L}, k_{L}\right)\right), \theta_{i}\right)\right] \\
& \Leftrightarrow \frac{1}{2}-\mu^{2}\left(\frac{1}{2}-\lambda\right)>1-\mu+\lambda(2 \mu-1) \Leftrightarrow \mu-\mu^{2}\left(\frac{1}{2}-\lambda\right)>\frac{1}{2}+\lambda(2 \mu-1) \Leftrightarrow \lambda>\frac{1}{2},
\end{aligned}
$$

$$
\begin{aligned}
& \mathbb{E}_{\theta, k}\left[u_{i}\left(\left(Q\left(\theta_{H}, k_{L}\right) Q\left(\theta_{L}, k_{L}\right), Q\left(\theta_{H}, k_{H}\right) Q\left(\theta_{L}, k_{L}\right)\right), \theta_{i}\right)\right] \\
&>\mathbb{E}_{\theta, k}\left[u_{i}\left(\left(Q\left(\theta_{H}, k_{H}\right) Q\left(\theta_{L}, k_{L}\right), Q\left(\theta_{H}, k_{H}\right) Q\left(\theta_{L}, k_{L}\right)\right), \theta_{i}\right)\right] \\
& \Leftrightarrow \frac{1}{2}-\mu^{2}\left(\frac{1}{2}-\lambda\right)>\frac{1}{2} \Leftrightarrow 0>\mu^{2}\left(\frac{1}{2}-\lambda\right) \Leftrightarrow \lambda>\frac{1}{2}
\end{aligned}
$$


and

$$
\begin{aligned}
\mathbb{E}_{\theta, k}\left[u_{i}\left(\left(Q\left(\theta_{H}, k_{L}\right) Q\left(\theta_{L}, k_{L}\right), Q\left(\theta_{H}, k_{H}\right) Q\left(\theta_{L}, k_{L}\right)\right), \theta_{i}\right)\right] & \\
>\mathbb{E}_{\theta, k}\left[u_{i}\left(\left(Q\left(\theta_{H}, k_{H}\right) Q\left(\theta_{L}, k_{H}\right), Q\left(\theta_{H}, k_{H}\right) Q\left(\theta_{L}, k_{L}\right)\right), \theta_{i}\right)\right] & \Leftrightarrow \frac{1}{2}-\mu^{2}\left(\frac{1}{2}-\lambda\right)>1-\lambda+\mu\left(\frac{\mu}{2}+2 \lambda-\mu \lambda-1\right) \Leftrightarrow \lambda>\frac{1}{2} .
\end{aligned}
$$

Conditions (84)-(86) are satisfied by the postulate of Theorem 2 that $2 \lambda-1>\mu>2(1-\lambda)$.

Finally, suppose now that player $j$ uses the strategy $\sigma_{j}\left(\theta_{j}\right)=\left(Q\left(\theta_{H}, k_{H}\right) Q\left(\theta_{L}, k_{H}\right)\right)$. Player $i$ 's strategy is a best response to this if

$$
\begin{gathered}
\mathbb{E}_{\theta, k}\left[u_{i}\left(\left(Q\left(\theta_{H}, k_{L}\right) Q\left(\theta_{L}, k_{L}\right), Q\left(\theta_{H}, k_{H}\right) Q\left(\theta_{L}, k_{H}\right)\right), \theta_{i}\right)\right] \\
>\mathbb{E}_{\theta, k}\left[u_{i}\left(\left(Q\left(\theta_{H}, k_{L}\right) Q\left(\theta_{L}, k_{H}\right), Q\left(\theta_{H}, k_{H}\right) Q\left(\theta_{L}, k_{H}\right)\right), \theta_{i}\right)\right] \\
\Leftrightarrow \lambda(1-\mu(1-\mu))>\frac{1}{2}-\mu\left(1-\frac{1}{2}-\lambda\right) \Leftrightarrow \lambda>\frac{1}{2}, \\
\mathbb{E}_{\theta, k}\left[u_{i}\left(\left(Q\left(\theta_{H}, k_{L}\right) Q\left(\theta_{L}, k_{L}\right), Q\left(\theta_{H}, k_{H}\right) Q\left(\theta_{L}, k_{H}\right)\right), \theta_{i}\right)\right] \\
>\mathbb{E}_{\theta, k}\left[u_{i}\left(\left(Q\left(\theta_{H}, k_{L}\right) Q\left(\theta_{L}, k_{H}\right), Q\left(\theta_{H}, k_{H}\right) Q\left(\theta_{L}, k_{H}\right)\right), \theta_{i}\right)\right] \\
\Leftrightarrow \lambda(1-\mu(1-\mu))>\lambda-\mu\left(\frac{\mu}{2}+2 \lambda-\mu \lambda-1\right) \Leftrightarrow \lambda>\frac{1}{2} \text { and } \mu>2(1-\lambda)
\end{gathered}
$$

and

$$
\begin{gathered}
\mathbb{E}_{\theta, k}\left[u_{i}\left(\left(Q\left(\theta_{H}, k_{L}\right) Q\left(\theta_{L}, k_{L}\right), Q\left(\theta_{H}, k_{H}\right) Q\left(\theta_{L}, k_{H}\right)\right), \theta_{i}\right)\right] \\
>\mathbb{E}_{\theta, k}\left[u_{i}\left(\left(Q\left(\theta_{H}, k_{H}\right) Q\left(\theta_{L}, k_{H}\right), Q\left(\theta_{H}, k_{H}\right) Q\left(\theta_{L}, k_{H}\right)\right), \theta_{i}\right)\right] \\
\Leftrightarrow \lambda(1-\mu(1-\mu))>\frac{1}{2} \Leftrightarrow \lambda>\frac{2}{3} .
\end{gathered}
$$

Conditions (86)-(89) are satisfied by the postulate of Theorem 2 that $2 \lambda-1>\mu>2(1-\lambda)$.

Therefore, I have shown that, irrespective of player $j$ 's strategy, player $i$ 's best response is $\sigma_{i}\left(\theta_{i}\right)=\left(Q\left(\theta_{H}, k_{L}\right) Q\left(\theta_{L}, k_{L}\right)\right)$. As $i, j \in \mathscr{I}$ were assigned arbitrarily and the players' payoffs are symmetric this implies that the strategy profile $\sigma^{*}=\left(Q\left(\theta_{H}, k_{L}\right) Q\left(\theta_{L}, k_{L}\right), Q\left(\theta_{H}, k_{L}\right) Q\left(\theta_{L}, k_{L}\right)\right)$ is the unique pure strategy Bayes-Nash equilibrium of the game in Figure 12. I have also demonstrated that the postulate of Theorem 1 that $2 \lambda-1>\mu>2(1-\lambda)$ is a sufficient condition for the existence of this equilibrium. This completes the proof of Theorem 2.

Proof of Proposition 5. Under Assumption 1 and Assumption 2 the strategic form of the game when $\theta_{1}=\theta_{2}$ is common knowledge is given in Figure 13 . 


\begin{tabular}{|c|c|c|}
\hline & $Q\left(\theta_{2}, k_{L}\right)$ & $Q\left(\theta_{2}, k_{H}\right)$ \\
\hline$Q\left(\theta_{1}, k_{L}\right)$ & $\frac{1}{2}, \frac{1}{2}$ & $\lambda, 1-\lambda$ \\
\hline$Q\left(\theta_{1}, k_{H}\right)$ & $1-\lambda, \lambda$ & $\frac{1}{2}, \frac{1}{2}$ \\
\hline
\end{tabular}

Figure 13: Strategic form when types are common knowledge and $\theta_{1}=\theta_{2}$.

It is clear from Figure 13 that the value of $\operatorname{Pr}\left(k=k_{L}\right)=\lambda$ is salient in the determination of equilibrium. Therefore, I will consider two distinct cases. Suppose first that $\operatorname{Pr}\left(k=k_{L}\right)>$ $\operatorname{Pr}\left(k=k_{H}\right)$, then, each player $i \in \mathscr{I}$ has a dominant strategy in $Q\left(\theta_{i}, k_{L}\right)$ as

$$
\mathbb{E}_{k}\left[u_{i}\left(\left(Q\left(\theta_{i}, k_{L}\right), Q\left(\theta_{j}, k_{L}\right)\right), \theta_{i}\right)\right]>\mathbb{E}_{k}\left[u_{i}\left(\left(Q\left(\theta_{i}, k_{H}\right), Q\left(\theta_{j}, k_{L}\right)\right), \theta_{i}\right)\right]
$$

and

$$
\mathbb{E}_{k}\left[u_{i}\left(\left(Q\left(\theta_{i}, k_{L}\right), Q\left(\theta_{j}, k_{H}\right)\right), \theta_{i}\right)\right]>\mathbb{E}_{k}\left[u_{i}\left(\left(Q\left(\theta_{i}, k_{H}\right), Q\left(\theta_{j}, k_{H}\right)\right), \theta_{i}\right)\right]
$$

The dominance solvable Nash equilibrium is, therefore, $\sigma^{*}=\left(Q\left(\theta_{1}, k_{L}\right), Q\left(\theta_{2}, k_{L}\right)\right)$.

Next, suppose that $\operatorname{Pr}\left(k=k_{H}\right)>\operatorname{Pr}\left(k=k_{L}\right)$, then, each player $i \in \mathscr{I}$ has a dominant strategy in $Q\left(\theta_{i}, k_{H}\right)$ as

$$
\mathbb{E}_{k}\left[u_{i}\left(\left(Q\left(\theta_{i}, k_{H}\right), Q\left(\theta_{j}, k_{L}\right)\right), \theta_{i}\right)\right]>\mathbb{E}_{k}\left[u_{i}\left(\left(Q\left(\theta_{i}, k_{L}\right), Q\left(\theta_{j}, k_{L}\right)\right), \theta_{i}\right)\right]
$$

and

$$
\mathbb{E}_{k}\left[u_{i}\left(\left(Q\left(\theta_{i}, k_{H}\right), Q\left(\theta_{j}, k_{H}\right)\right), \theta_{i}\right)\right]>\mathbb{E}_{k}\left[u_{i}\left(\left(Q\left(\theta_{i}, k_{L}\right), Q\left(\theta_{j}, k_{H}\right)\right), \theta_{i}\right)\right]
$$

The dominance solvable Nash equilibrium is, therefore, $\sigma^{*}=\left(Q\left(\theta_{1}, k_{H}\right), Q\left(\theta_{2}, k_{H}\right)\right)$. This completes the proof of Proposition 5.

Proof of Proposition 6. Recall that $\operatorname{Pr}\left(k=k_{L}\right)=\lambda$. Then, under Assumption 1 and Assumption 2, the strategic form when $\theta_{1}=\theta_{H}>\theta_{L}=\theta_{L}$ is common knowledge is given in Figure 14 .

2

\begin{tabular}{cc|c|c|} 
& & $Q\left(\theta_{2}, k_{L}\right)$ & $Q\left(\theta_{2}, k_{H}\right)$ \\
\cline { 3 - 4 } & $Q\left(\theta_{1}, k_{L}\right)$ & $\underline{1}, 0$ & $\lambda, \underline{1-\lambda}$ \\
\cline { 3 - 4 } & & & \\
\cline { 3 - 4 } & $Q\left(\theta_{1}, k_{H}\right)$ & $1-\lambda, \underline{\lambda}$ & $\underline{1}, 0$ \\
\cline { 2 - 3 } & &
\end{tabular}

Figure 14: Strategic form when types are common knowledge and $\theta_{1}>\theta_{2}$. 
Hence, there are no equilibria in pure strategies and so I look for equilibria in mixed strategies; that is, a strategy $\hat{\sigma}_{i}\left(\theta_{i}\right) \in \Delta(\mathscr{T})$ for each $i \in \mathscr{I}$ such that $\hat{\sigma}=\left(\hat{\sigma_{1}}, \hat{\sigma_{2}}\right)$ constitutes a Nash equilibrium. Suppose that $\operatorname{Pr}\left[\sigma_{1}\left(\theta_{1}\right)=Q\left(\theta_{1}, k_{L}\right)\right]:=p \in(0,1)$ and $\operatorname{Pr}\left[\sigma_{2}\left(\theta_{2}\right)=Q\left(\theta_{2}, k_{L}\right)\right]:=$ $q \in(0,1)$. These mixing probabilities are is illustrated in Figure 15. A mixed strategy of player 1

\begin{tabular}{|c|c|c|}
\hline & \multicolumn{2}{|c|}{2} \\
\hline & $Q\left(\theta_{2}, k_{L}\right)(q)$ & $Q\left(\theta_{2}, k_{H}\right)(1-q)$ \\
\hline$Q\left(\theta_{1}, k_{L}\right)(p)$ & 1,0 & $\lambda, 1-\lambda$ \\
\hline$Q\left(\theta_{1}, k_{H}\right)(1-p)$ & $1-\lambda, \lambda$ & 1,0 \\
\hline
\end{tabular}

Figure 15: Mixing probabilities when types are common knowledge and $\theta_{1}>\theta_{2}$.

will constitute part of a mixed strategy Nash equilibrium if it makes player 2 indifferent between her pure strategies. Hence, player 1 will choose $p$ such that

$$
\mathbb{E}_{k}\left[u_{2}\left(\left(\hat{\sigma}\left(\theta_{1}\right), Q\left(\theta_{2}, k_{L}\right)\right), \theta_{2}\right)\right]=\mathbb{E}_{k}\left[u_{2}\left(\left(\hat{\sigma}\left(\theta_{1}\right), Q\left(\theta_{2}, k_{L}\right)\right), \theta_{2}\right)\right] .
$$

Condition (90) is equivalent to

$$
p \cdot 0+(1-p) \cdot \lambda=p \cdot(1-\lambda)+(1-p) \cdot 0 \Rightarrow p^{*}=\operatorname{Pr}\left(k=k_{L}\right) .
$$

Analogously, a mixed strategy of player 2 will constitute part of a mixed strategy Nash equilibrium if it makes player 1 indifferent between her pure strategies. Hence, player 2 will choose $q$ such that

$$
\mathbb{E}_{k}\left[u_{1}\left(\left(Q\left(\theta_{1}, k_{L}\right), \hat{\sigma}\left(\theta_{2}\right)\right), \theta_{1}\right)\right]=\mathbb{E}_{k}\left[u_{1}\left(\left(Q\left(\theta_{1}, k_{H}\right), \hat{\sigma}\left(\theta_{2}\right)\right), \theta_{1}\right)\right] .
$$

Condition (91) is equivalent to

$$
q \cdot 1+(1-q) \cdot \lambda=q \cdot(1-\lambda)+(1-q) \cdot 1 \Rightarrow q^{*}=1-\operatorname{Pr}\left(k=k_{L}\right)=\operatorname{Pr}\left(k=k_{H}\right) .
$$

The mixed strategy Nash equilibrium is therefore $\hat{\sigma}^{*}=\left(\operatorname{Pr}\left(k=k_{L}\right) Q\left(\theta_{1}, k_{L}\right), \operatorname{Pr}\left(k=k_{H}\right) Q\left(\theta_{2}, k_{L}\right)\right)$. This completes the proof of Proposition 6.

Proof of Proposition 8. To prove this result I will focus on symmetric equilibria in which each player uses the same strategy. Before demonstrating that this result holds for all $\sigma_{i}\left(\theta_{i}\right) \in \mathscr{T}$, I will analyse the case when $\sigma_{i}\left(\theta_{i}\right) \in\left\{\theta_{i}-\gamma_{L}, \theta_{i}, \theta_{i}+\gamma_{H}\right\}$ and then extend my analysis to the continuum case. Throughout the proof I will assume that $\operatorname{Pr}\left(k=k_{L}\right)=1$, which is without loss of generality as analogous results hold when $\operatorname{Pr}\left(k=k_{L}\right)=0$.

The first case I will consider is when $\gamma:=\underline{\gamma}_{i}=\bar{\gamma}_{i}=\underline{\gamma}_{j}=\bar{\gamma}_{j}>0$ for all $i, j \in \mathscr{I}$. For 
notational convenience I will label each strategy as

$$
\begin{aligned}
\sigma_{i}^{1}\left(\theta_{i}\right) & =\theta_{i}, \\
\sigma_{i}^{2}\left(\theta_{i}\right) & =\theta_{i}-\gamma, \\
\sigma_{i}^{3}\left(\theta_{i}\right) & =\theta_{i}+\gamma
\end{aligned}
$$

I will now show that $\sigma_{i}^{1}\left(\theta_{i}\right)$ is a dominant strategy for each player $i \in \mathscr{I}$. Suppose first that this is true, then, the strategy profile $\sigma^{1}=\left(\sigma_{1}^{1}\left(\theta_{1}\right), \ldots, \sigma_{N}^{1}\left(\theta_{N}\right)\right.$ is the unique pure strategy Bayes-Nash equilibrium. At this strategy profile each player $i$ has the expected payoff

$$
\begin{array}{r}
\mathbb{E}_{\theta}\left[u_{i}\left(\sigma^{1}, \theta_{i}\right)\right]=\int_{\Theta_{-i}} \operatorname{Pr}\left(S\left(Q\left(\theta_{i}, k_{L}\right), \sigma_{i}^{1}\left(\theta_{i}\right)>S\left(Q\left(\theta_{j}, k_{L}\right), \sigma_{j}^{1}\left(\theta_{j}\right)\right) \forall j \neq i\right) d \mathcal{F}\left(\theta_{-i}\right)\right. \\
=\operatorname{Pr}\left(\theta_{i}>\theta_{j} \forall j \neq i\right)=\left(\mathcal{F}\left(\theta_{i}\right)\right)^{N-1},
\end{array}
$$

which is equal to the second highest order statistic of the distribution of the players' abilities. Player $i$ 's expected probability of winning the contest is, therefore, equal to the probability that the $N-1$ competitors she faces each have a lower ability than she has. I will show that player $i$ has no incentive to deviate from this equilibrium. If she deviates to $\sigma_{i}^{2}\left(\theta_{i}\right)$ her expected payoff is

$$
\mathbb{E}_{\theta}\left[u_{i}\left(\left(\sigma_{i}^{2}\left(\theta_{i}\right), \sigma^{1} \backslash\left\{\sigma_{i}\left(\theta_{i}\right)\right\}\right), \theta_{i}\right)\right]=\operatorname{Pr}\left(\theta_{i}-\gamma>\theta_{j} \forall j \neq i\right)=\left(\mathcal{F}\left(\theta_{i}-\gamma\right)\right)^{N-1},
$$

where $\sigma^{1} \backslash\left\{\sigma_{i}\left(\theta_{i}\right)\right\}=\left(\sigma_{1}^{1}\left(\theta_{1}\right), \ldots, \sigma_{i-1}^{1}\left(\theta_{i-1}\right), \sigma_{i+1}^{1}\left(\theta_{i+1}\right), \ldots, \sigma_{N}^{1}\left(\theta_{N}\right)\right.$. Whilst, if she deviates to $\sigma_{i}^{3}\left(\theta_{i}\right)$ her expected payoff is

$$
\begin{aligned}
\mathbb{E}_{\theta}\left[u_{i}\left(\left(\sigma_{i}^{3}\left(\theta_{i}\right), \sigma^{1} \backslash\left\{\sigma_{i}\left(\theta_{i}\right)\right\}\right), \theta_{i}\right)\right] & =\operatorname{Pr}\left(\theta_{i}+\gamma+\delta\left(\theta_{i}+\gamma-\theta_{i}\right)>\theta_{j} \forall j \neq i\right) \\
=\operatorname{Pr}\left(\theta_{i}-\gamma(\delta-1)>\theta_{j} \forall j \neq i\right) & =\left(\mathcal{F}\left(\theta_{i}-\gamma(\delta-1)\right)\right)^{N-1} .
\end{aligned}
$$

Equations (92)-(94) are consequences of the properties of the scoring rule (2). Comparing these expected payoffs shows that there is no profitable deviation as

$$
\mathcal{F}\left(\theta_{i}\right)>\mathcal{F}\left(\theta_{i}-\gamma(\delta-1)\right)>\mathcal{F}\left(\theta_{i}-\gamma\right)
$$

Condition (95) holds because $\mathcal{F}$ is nondecreasing by definition, $\gamma>0$ by construction and $\delta>1$ by Lemma 1 . To see why this result holds for any $\sigma_{i}\left(\theta_{i}\right) \in\left[\theta_{i}-\gamma, \theta_{i}+\gamma\right]$ consider that for any $\beta_{1}, \beta_{2} \in(0,1)$ I have, irrespective of whether $\beta_{1}=\beta_{2}$ or $\beta_{1} \neq \beta_{2}$,

$$
\mathcal{F}\left(\theta_{i}\right)>\mathcal{F}\left(\theta_{i}-\beta_{1} \gamma(\delta-1)\right) \text { and } \mathcal{F}\left(\theta_{i}\right)>\mathcal{F}\left(\theta_{i}-\beta_{2} \gamma\right)
$$

In fact, a condition analogous to (95) will hold for any $\frac{\beta_{1}+\beta_{2}}{\beta 1}>\delta>1$, but this is not necessary for my result.

I now show that there exists a profitable deviation for player $i$ when all her competitors use the strategy $\sigma_{j}^{2}\left(\theta_{j}\right)$ for $j \neq i$. Suppose that the strategy profile $\sigma^{2}$ is a Bayes-Nash equilibrium. 
Then, by following similar methods as the derivation of (92), I find that every player has an expected payoff of $\left(\mathcal{F}\left(\theta_{i}\right)\right)^{N-1}$. If player $i$ deviates to the strategy $\sigma_{i}^{1}\left(\theta_{i}\right)$ she obtains an expected payoff of

$$
\begin{aligned}
\mathbb{E}_{\theta}\left[u_{i}\left(\left(\sigma_{i}^{1}\left(\theta_{i}\right), \sigma^{2} \backslash\left\{\sigma_{i}\left(\theta_{i}\right)\right\}\right), \theta_{i}\right)\right]=\operatorname{Pr}\left(\theta_{i}>\theta_{j}\right. & -\gamma \forall j \neq i) \\
& =\operatorname{Pr}\left(\theta_{i}+\gamma>\theta_{j} \forall j \neq i\right)=\left(\mathcal{F}\left(\theta_{i}+\gamma\right)\right)^{N-1},
\end{aligned}
$$

which I derive by following similar methods to (93). Therefore, at this strategy profile, player $i$ has an incentive to deviate to the strategy $\sigma_{i}^{1}\left(\theta_{i}\right)$ as

$$
\mathcal{F}\left(\theta_{i}+\gamma\right)>\mathcal{F}\left(\theta_{i}\right)
$$

Finally, I show that there exists a profitable deviation for player $i$ when all her competitors use the strategy $\sigma_{j}^{3}\left(\theta_{j}\right)$ for $j \neq i$. Suppose that the strategy profile $\sigma^{3}$ is a Bayes-Nash equilibrium. Once again, each player has an expected payoff of $\left(\mathcal{F}\left(\theta_{i}\right)\right)^{N-1}$. If player $i$ deviates to the strategy $\sigma_{i}^{1}\left(\theta_{i}\right)$ she obtains an expected payoff of

$$
\begin{aligned}
\mathbb{E}_{\theta}\left[u_{i}\left(\left(\sigma_{i}^{1}\left(\theta_{i}\right), \sigma^{3} \backslash\left\{\sigma_{i}\left(\theta_{i}\right)\right\}\right), \theta_{i}\right)\right] & =\operatorname{Pr}\left(\theta_{i}>\theta_{j}-\gamma(\delta-1) \forall j \neq i\right) \\
& =\operatorname{Pr}\left(\theta_{i}+\gamma(\delta-1)>\theta_{j} \forall j \neq i\right)=\left(\mathcal{F}\left(\theta_{i}+\gamma(\delta-1)\right)\right)^{N-1},
\end{aligned}
$$

which I derive by following similar methods to (94). At this strategy profile player $i$ has an incentive to deviate to the strategy $\sigma_{i}^{1}\left(\theta_{i}\right)$ as

$$
\mathcal{F}\left(\theta_{i}+\gamma(\delta-1)\right)>\mathcal{F}\left(\theta_{i}\right)
$$

As before, I can show that this can be generalised to the continuum strategy space by introducing $\beta_{1}$ and $\beta_{2}$. In this case, (96) and (97) become $\mathcal{F}\left(\theta_{i}+\beta_{1} \gamma\right)>\mathcal{F}\left(\theta_{i}\right)$ and $\mathcal{F}\left(\theta_{i}+\beta_{2} \gamma(\delta-1)\right)>\mathcal{F}\left(\theta_{i}\right)$, respectively.

I have shown that, since there is an incentive to deviate for any strategy other than $\sigma_{i}^{1}\left(\theta_{i}\right)$ for each $i \in \mathscr{I}$, this strategy constitutes a dominant strategy. The strategy profile $\sigma^{1}$ is, therefore, the unique dominance solvable pure strategy Bayes-Nash equilibrium when $\gamma:=\underline{\gamma}_{i}=\bar{\gamma}_{i}=\underline{\gamma}_{j}=$ $\bar{\gamma}_{j}>0$.

I now consider the case when $\bar{\gamma}_{i}>\underline{\gamma}_{i}>0$ for each $i \in \mathscr{I}$ in addition to $\bar{\gamma}_{i} \neq \bar{\gamma}_{j}$ and $\underline{\gamma}_{i} \neq \underline{\gamma}_{j}$ for $i \neq j \in \mathscr{I}$. For this more general setting I label the strategies

$$
\begin{aligned}
& \tilde{\sigma}_{i}^{1}\left(\theta_{i}\right)=\theta_{i}, \\
& \tilde{\sigma}_{i}^{2}\left(\theta_{i}\right)=\theta_{i}-\underline{\gamma}_{i}, \\
& \tilde{\sigma}_{i}^{3}\left(\theta_{i}\right)=\theta_{i}+\bar{\gamma}_{i} .
\end{aligned}
$$

As in the first half of the proof, I first assume that the strategy profile $\tilde{\sigma}^{1}$ is the unique pure strategy Bayes-Nash equilibrium and demonstrate that player $i$ has no incentive to deviate. At this strategy profile each player $i \in \mathscr{I}$ has, analogously to (92), an expected payoff of $\left(\mathcal{F}\left(\theta_{i}\right)\right)^{N-1}$. 
If she now deviates to $\tilde{\sigma}_{i}^{2}\left(\theta_{i}\right)$ she obtains an expected payoff of $\left(\mathcal{F}\left(\theta_{i}-\underline{\gamma}_{i}\right)\right)^{N-1}$. Whilst, if she deviates to $\tilde{\sigma}_{i}^{3}\left(\theta_{i}\right)$, her expected payoff is $\left(\mathcal{F}\left(\theta_{i}-\bar{\gamma}_{i}(\delta-1)\right)\right)^{N-1}$. Therefore, player $i$ has no incentive to deviate from the proposed equilibrium strategy profile $\tilde{\sigma}^{1}$ as

$$
\mathcal{F}\left(\theta_{i}\right)>\mathcal{F}\left(\theta_{i}-\underline{\gamma}_{i}\right) \text { and } \mathcal{F}\left(\theta_{i}\right)>\mathcal{F}\left(\theta_{i}-\bar{\gamma}_{i}(\delta-1)\right)
$$

Suppose instead that the strategy profile $\tilde{\sigma}^{2}$ is the Bayes-Nash equilibrium. In this case player $i$ has an expected payoff of

$$
\begin{aligned}
\mathbb{E}_{\theta}\left[u_{i}\left(\tilde{\sigma}^{2}, \theta_{i}\right)\right] & =\operatorname{Pr}\left(\theta_{i}-\underline{\gamma}_{i}>\theta_{j}-\underline{\gamma}_{j} \forall j \neq i\right)=\operatorname{Pr}\left(\theta_{i}-\underline{\gamma}_{i}+\underline{\gamma}_{j}>\theta_{j} \forall j \neq i\right) \\
& =\mathcal{F}\left(\theta_{i}-\underline{\gamma}_{i}+\underline{\gamma}_{1}\right) \ldots \mathcal{F}\left(\theta_{i}-\underline{\gamma}_{i}+\underline{\gamma}_{i-1}\right) \mathcal{F}\left(\theta_{i}-\underline{\gamma}_{i}+\underline{\gamma}_{i+1}\right) \ldots \mathcal{F}\left(\theta_{i}-\underline{\gamma}_{i}+\underline{\gamma}_{N}\right) .
\end{aligned}
$$

If she deviates to $\tilde{\sigma}_{i}^{1}\left(\theta_{i}\right)$ she can obtain an expected payoff of

$$
\begin{aligned}
\mathbb{E}_{\theta}\left[u_{i}\left(\left(\tilde{\sigma}_{i}^{1}\left(\theta_{i}\right), \tilde{\sigma}^{2} \backslash\left\{\sigma_{i}\left(\theta_{i}\right)\right\}\right), \theta_{i}\right)\right] & \\
=\operatorname{Pr}\left(\theta_{i}>\theta_{j}-\right. & \left.\underline{\gamma}_{j} \forall j \neq i\right)=\operatorname{Pr}\left(\theta_{i}+\underline{\gamma}_{j}>\theta_{j} \forall j \neq i\right) \\
& =\mathcal{F}\left(\theta_{i}+\underline{\gamma}_{1}\right) \ldots \mathcal{F}\left(\theta_{i}+\underline{\gamma}_{i-1}\right) \mathcal{F}\left(\theta_{i}+\underline{\gamma}_{i+1}\right) \ldots \mathcal{F}\left(\theta_{i}+\underline{\gamma}_{N}\right) .
\end{aligned}
$$

This deviation is profitable as

$$
\begin{aligned}
\mathcal{F}\left(\theta_{i}+\underline{\gamma}_{1}\right) & \cdots \mathcal{F}\left(\theta_{i}+\underline{\gamma}_{i-1}\right) \mathcal{F}\left(\theta_{i}+\underline{\gamma}_{i+1}\right) \cdots \mathcal{F}\left(\theta_{i}+\underline{\gamma}_{N}\right) \\
& >\mathcal{F}\left(\theta_{i}-\underline{\gamma}_{i}+\underline{\gamma}_{1}\right) \ldots \mathcal{F}\left(\theta_{i}-\underline{\gamma}_{i}+\underline{\gamma}_{i-1}\right) \mathcal{F}\left(\theta_{i}-\underline{\gamma}_{i}+\underline{\gamma}_{i+1}\right) \cdots \mathcal{F}\left(\theta_{i}-\underline{\gamma}_{i}+\underline{\gamma}_{N}\right) .
\end{aligned}
$$

Finally, suppose that the strategy profile $\tilde{\sigma}^{3}$ is the Bayes-Nash equilibrium. In this case player $i$ has an expected payoff of

$$
\begin{aligned}
& \mathbb{E}_{\theta}\left[u_{i}\left(\tilde{\sigma}^{3}, \theta_{i}\right)\right] \\
& =\operatorname{Pr}\left(\theta_{i}-\underline{\gamma}_{i}(\delta-1)>\theta_{j}-\underline{\gamma}_{j}(\delta-1) \forall j \neq i\right)=\operatorname{Pr}\left(\theta_{i}-\left(\underline{\gamma}_{i}-\underline{\gamma}_{j}\right)(\delta-1)>\theta_{j} \forall j \neq i\right) \\
& =\mathcal{F}\left(\theta_{i}-\left(\underline{\gamma}_{i}-\underline{\gamma}_{1}\right)(\delta-1)\right) \ldots \mathcal{F}\left(\theta_{i}-\left(\underline{\gamma}_{i}-\underline{\gamma}_{i-1}\right)(\delta-1)\right) \times \\
& \quad \times \mathcal{F}\left(\theta_{i}-\left(\underline{\gamma}_{i}-\underline{\gamma}_{i+1}\right)(\delta-1)\right) \ldots \mathcal{F}\left(\theta_{i}-\left(\underline{\gamma}_{i}-\underline{\gamma}_{N}\right)(\delta-1)\right) .
\end{aligned}
$$

If she deviates to $\tilde{\sigma}_{i}^{1}\left(\theta_{i}\right)$ she can obtain an expected payoff of

$$
\begin{aligned}
& \mathbb{E}_{\theta}\left[u_{i}\left(\left(\tilde{\sigma}_{i}^{1}\left(\theta_{i}\right), \tilde{\sigma}^{3} \backslash\left\{\sigma_{i}\left(\theta_{i}\right)\right\}\right), \theta_{i}\right)\right] \\
& \quad=\operatorname{Pr}\left(\theta_{i}>\theta_{j}-\underline{\gamma}_{j}(\delta-1) \forall j \neq i\right)=\operatorname{Pr}\left(\theta_{i}+\underline{\gamma}_{j}(\delta-1)>\theta_{j} \forall j \neq i\right) \\
& =\mathcal{F}\left(\theta_{i}+\underline{\gamma}_{1}(\delta-1)\right) \cdots \mathcal{F}\left(\theta_{i}+\underline{\gamma}_{i-1}(\delta-1)\right) \mathcal{F}\left(\theta_{i}+\underline{\gamma}_{i+1}(\delta-1)\right) \cdots \mathcal{F}\left(\theta_{i}+\underline{\gamma}_{N}(\delta-1)\right) .
\end{aligned}
$$


This deviation is profitable as

$$
\begin{aligned}
& \mathcal{F}\left(\theta_{i}+\underline{\gamma}_{1}(\delta-1)\right) \cdots \mathcal{F}\left(\theta_{i}+\underline{\gamma}_{i-1}(\delta-1)\right) \mathcal{F}\left(\theta_{i}+\underline{\gamma}_{i+1}(\delta-1)\right) \cdots \mathcal{F}\left(\theta_{i}+\underline{\gamma}_{N}(\delta-1)\right)> \\
& \mathcal{F}\left(\theta_{i}-\left(\underline{\gamma}_{i}-\underline{\gamma}_{1}\right)(\delta-1)\right) \cdots \mathcal{F}\left(\theta_{i}-\left(\underline{\gamma}_{i}-\underline{\gamma}_{i-1}\right)(\delta-1)\right) \times \\
& \quad \times \mathcal{F}\left(\theta_{i}-\left(\underline{\gamma}_{i}-\underline{\gamma}_{i+1}\right)(\delta-1)\right) \cdots \mathcal{F}\left(\theta_{i}-\left(\underline{\gamma}_{i}-\underline{\gamma}_{N}\right)(\delta-1)\right) .
\end{aligned}
$$

I have shown that, since there is an incentive to deviate for any strategy other than $\tilde{\sigma}_{i}^{1}\left(\theta_{i}\right)$ for each $i \in \mathscr{I}$, this strategy constitutes a dominant strategy. The strategy profile $\tilde{\sigma}^{1}$ is, therefore, the unique dominance solvable pure strategy Bayes-Nash equilibrium when $\bar{\gamma}_{i}>\underline{\gamma}_{i}>0$ for each $i \in \mathscr{I}$ in addition to $\bar{\gamma}_{i} \neq \bar{\gamma}_{j}$ and $\underline{\gamma}_{i} \neq \underline{\gamma}_{j}$ for $i \neq j \in \mathscr{I}$. As I have considered strategies on the bounds of the strategy space, it is clear that conditions (98) and (99) will hold for any strategy $\sigma_{i}\left(\theta_{i}\right) \in \operatorname{int}(\mathscr{T})$ and, hence, the strategy $\sigma_{i}\left(\theta_{i}\right)=\theta_{i}$ will always constitute a dominant strategy. This completes the proof of Proposition 8.

Proof of Proposition 9. I begin by deriving the two key conditions highlighted in the main text: (9) and (10). I will then prove the result in Example 1. First, I will show, in a manner akin to the proof of Proposition 8, that each player has a dominant strategy. Given that there are now two states of nature each player's expected payoff is

$$
\begin{array}{r}
\mathbb{E}_{\theta, k}\left[u_{i}\left(\sigma, \theta_{i}\right)\right] \\
\quad=\sum_{k \in \mathscr{K}} \int_{\Theta_{-i}} \operatorname{Pr}\left(S\left(Q\left(\theta_{i}, k_{L}\right), \sigma_{i}\left(\theta_{i}\right)>S\left(Q\left(\theta_{j}, k_{L}\right), \sigma_{j}\left(\theta_{j}\right)\right) \forall j \neq i\right) \rho(k) d \mathcal{F}\left(\theta_{-i}\right) .\right.
\end{array}
$$

Again, to simplify notation, I will label player $i$ 's strategies as

$$
\sigma_{i}^{\prime}\left(\theta_{i}\right)=Q\left(\theta_{i}, k_{L}\right) \text { and } \sigma_{i}^{\prime \prime}\left(\theta_{i}\right)=Q\left(\theta_{i}, k_{H}\right)
$$

Suppose first that $\sigma^{\prime}=\left(\sigma_{1}^{\prime}\left(\theta_{1}\right), \ldots, \sigma_{N}^{\prime}\left(\theta_{N}\right)\right)$ is a Bayes-Nash equilibrium. Then, in this conjectured equilibrium, each player has an expected payoff of

$$
\begin{aligned}
& \mathbb{E}_{\theta, k}\left[u_{i}\left(\sigma^{\prime}, \theta_{i}\right)\right] \\
& \quad=\operatorname{Pr}\left(k=k_{L}\right) \operatorname{Pr}\left(\theta_{i}>\theta_{j} \forall j \neq i\right)+\operatorname{Pr}\left(k=k_{H}\right) \operatorname{Pr}\left(\theta_{i}>\theta_{j} \forall j \neq i\right)=\left(\mathcal{F}\left(\theta_{i}\right)\right)^{N-1},
\end{aligned}
$$

as $\operatorname{Pr}\left(k=k_{H}\right)=1-\operatorname{Pr}\left(k=k_{L}\right)$. Now consider that player $i$ deviates to $\sigma_{i}^{\prime \prime}\left(\theta_{i}\right)$, her expected payoff becomes

$$
\begin{gathered}
\mathbb{E}_{\theta, k}\left[u_{i}\left(\left(\sigma_{i}^{\prime \prime}\left(\theta_{i}\right), \sigma^{\prime} \backslash\left\{\sigma_{i}\left(\theta_{i}\right)\right), \theta_{i}\right)\right]\right. \\
=\operatorname{Pr}\left(k=k_{L}\right) \operatorname{Pr}\left(\theta_{i}-k_{H}(\delta-1)>\theta_{j} \forall j \neq i\right)+\operatorname{Pr}\left(k=k_{H}\right) \operatorname{Pr}\left(\theta_{i}+k_{H}>\theta_{j} \forall j \neq i\right) \\
=\operatorname{Pr}\left(k=k_{L}\right)\left(\mathcal{F}\left(\theta_{i}-k_{H}(\delta-1)\right)\right)^{N-1}+\operatorname{Pr}\left(k=k_{H}\right)\left(\mathcal{F}\left(\theta_{i}+k_{H}\right)\right)^{N-1} .
\end{gathered}
$$


Comparing (100) and (101) provides the condition for there to be no profitable deviation, which is (9) in the main text,

$$
\begin{aligned}
\mathbb{E}_{\theta, k}\left[u_{i}\left(\sigma^{\prime}, \theta_{i}\right)\right]=\left(\mathcal{F}\left(\theta_{i}\right)\right)^{N-1}> & \\
\operatorname{Pr}\left(k=k_{L}\right)\left(\mathcal{F}\left(\theta_{i}-k_{H}(\delta-1)\right)\right)^{N-1}+ & \operatorname{Pr}\left(k=k_{H}\right)\left(\mathcal{F}\left(\theta_{i}+k_{H}\right)\right)^{N-1} \\
& =\mathbb{E}_{\theta, k}\left[u_{i}\left(\left(\sigma_{i}^{\prime \prime}\left(\theta_{i}\right), \sigma^{\prime} \backslash\left\{\sigma_{i}\left(\theta_{i}\right)\right\}\right), \theta_{i}\right)\right] .
\end{aligned}
$$

Suppose instead that $\sigma^{\prime \prime}=\left(\sigma_{1}^{\prime \prime}\left(\theta_{1}\right), \ldots, \sigma_{N}^{\prime \prime}\left(\theta_{N}\right)\right)$ is a Bayes-Nash equilibrium. Then, as in (100), each player has an expected payoff of

$$
\begin{aligned}
\mathbb{E}_{\theta, k} & {\left[u_{i}\left(\sigma^{\prime \prime}, \theta_{i}\right)\right] } \\
& =\operatorname{Pr}\left(k=k_{L}\right) \operatorname{Pr}\left(\theta_{i}>\theta_{j} \forall j \neq i\right)+\operatorname{Pr}\left(k=k_{H}\right) \operatorname{Pr}\left(\theta_{i}>\theta_{j} \forall j \neq i\right)=\left(\mathcal{F}\left(\theta_{i}\right)\right)^{N-1} .
\end{aligned}
$$

Now consider a deviation by player $i$ to $\sigma_{i}^{\prime}\left(\theta_{i}\right)$. This deviation yields her an expected payoff of

$$
\begin{aligned}
& \mathbb{E}_{\theta, k}\left[u_{i}\left(\left(\sigma_{i}^{\prime}\left(\theta_{i}\right), \sigma^{\prime \prime} \backslash\left\{\sigma_{i}\left(\theta_{i}\right)\right), \theta_{i}\right)\right]\right. \\
&=\operatorname{Pr}\left(k=k_{L}\right) \operatorname{Pr}\left(\theta_{i}+k_{H}(\delta-1)>\theta_{j} \forall j \neq i\right)+\operatorname{Pr}\left(k=k_{H}\right) \cdot \operatorname{Pr} .\left(\theta_{i}-k_{H}>\theta_{j} \forall j \neq i\right) . \\
&=\operatorname{Pr}\left(k=k_{L}\right)\left(\mathcal{F}\left(\theta_{i}+k_{H}(\delta-1)\right)\right)^{N-1}+\operatorname{Pr}\left(k=k_{H}\right)\left(\mathcal{F}\left(\theta_{i}-k_{H}\right)\right)^{N-1} .
\end{aligned}
$$

Comparing (103) and (104) provides the condition for there to be a profitable deviation, which is (10) in the main text,

$$
\begin{aligned}
& \mathbb{E}_{\theta, k}\left[u_{i}\left(\left(\sigma_{i}^{\prime}\left(\theta_{i}\right), \sigma^{\prime \prime} \backslash\left\{\sigma_{i}\left(\theta_{i}\right)\right\}\right), \theta_{i}\right)\right] \\
&=\operatorname{Pr}\left(k=k_{L}\right)\left(\mathcal{F}\left(\theta_{i}+k_{H}(\delta-1)\right)\right)^{N-1}+ \operatorname{Pr}\left(k=k_{H}\right)\left(\mathcal{F}\left(\theta_{i}-k_{H}\right)\right)^{N-1} \\
&>\left(\mathcal{F}\left(\theta_{i}\right)\right)^{N-1}=\mathbb{E}_{\theta, k}\left[u_{i}\left(\sigma^{\prime \prime}, \theta_{i}\right)\right]
\end{aligned}
$$

Conditions (102) and (105) are difficult to analyse in this general form as they depend on several relatively complicated objects. As such, to yield more tractable expressions, I will employ the conditions of Example 1 to simplify these terms. The assumption that there are two players implies that $N-1=1$, whilst the assumption of a uniform distribution of the players' abilities over the unit interval implies that $\mathcal{F}(x)=x$. These assumptions imply that (100) and (103) become

$$
\mathbb{E}_{\theta, k}\left[u_{i}\left(\sigma^{\prime}, \theta_{i}\right)\right]=\mathbb{E}_{\theta, k}\left[u_{i}\left(\sigma^{\prime \prime}, \theta_{i}\right)\right]=\theta_{i}
$$

Imposing these assumptions on (101) yields

$$
\begin{aligned}
\mathbb{E}_{\theta, k}\left[u_{i}\left(\left(\sigma_{i}^{\prime \prime}\left(\theta_{i}\right), \sigma^{\prime} \backslash\left\{\sigma_{i}\left(\theta_{i}\right)\right), \theta_{i}\right)\right]\right. & =\lambda \cdot F\left(\theta_{i}-k_{H}(\delta-1)\right)+(1-\lambda) \cdot F\left(\theta_{i}+k_{H}\right), \\
& =\lambda \int_{0}^{\theta_{i}-k_{H}(\delta-1)} d \theta_{j}+(1-\lambda) \int_{0}^{\theta_{i}+k_{H}} d \theta_{j}, \\
& =\theta_{i}+k_{H}(1-\lambda \delta) .
\end{aligned}
$$


Finally, applied to (104), these assumptions lead to

$$
\begin{aligned}
\mathbb{E}_{\theta, k}\left[u_{i}\left(\left(\sigma_{i}^{\prime}\left(\theta_{i}\right), \sigma^{\prime \prime} \backslash\left\{\sigma_{i}\left(\theta_{i}\right)\right), \theta_{i}\right)\right]\right. & =\lambda \cdot F\left(\theta_{i}+k_{H}(\delta-1)\right)+(1-\lambda) \cdot F\left(\theta_{i}-k_{H}\right), \\
& =\lambda \int_{0}^{\theta_{i}+k_{H}(\delta-1)} d \theta_{j}+(1-\lambda) \int_{0}^{\theta_{i}-k_{H}} d \theta_{j}, \\
& =\theta_{i}-k_{H}(1-\lambda \delta) .
\end{aligned}
$$

I can now express (102) in this more tractable form to determine the condition under which player $i$ will not have a profitable deviation from the strategy profile $\sigma^{\prime}$. This condition is

$$
\theta_{i}>\theta_{i}+k_{H}(1-\lambda \delta) \Leftrightarrow \delta>\frac{1}{\operatorname{Pr}\left(k=k_{L}\right)}
$$

which leads to the pure strategy Bayes-Nash equilibrium illustrated in Figure 16.

2

\begin{tabular}{cc|c|c|}
\multicolumn{2}{c}{$Q\left(\theta_{2}, k_{L}\right)$} & $Q\left(\theta_{2}, k_{H}\right)$ \\
\cline { 3 - 4 } & $Q\left(\theta_{1}, k_{L}\right)$ & $\underline{\theta_{1}}, \underline{\theta_{2}}$ & $\underline{\theta_{1}-k_{H}(1-\lambda \delta), \theta_{2}+k_{H}(1-\lambda \delta)}$ \\
\cline { 3 - 4 } & $Q\left(\theta_{1}, k_{H}\right)$ & $\theta_{1}+k_{H}(1-\lambda \delta), \underline{\theta_{2}-k_{H}(1-\lambda \delta)}$ & $\theta_{1}, \theta_{2}$ \\
\cline { 3 - 4 } & &
\end{tabular}

Figure 16: Equilibrium when $\delta>\frac{1}{\lambda}$.

Analogously, (105), the condition for player $i$ to have a profitable deviation from the strategy profile $\sigma^{\prime \prime}$ is

$$
\theta_{i}>\theta_{i}-k_{H}(1-\lambda \delta) \Leftrightarrow \delta<\frac{1}{\operatorname{Pr}\left(k=k_{L}\right)},
$$

which leads to the pure strategy Bayes-Nash equilibrium illustrated in Figure 17.

2

\begin{tabular}{cc|c|c|}
\multicolumn{2}{c}{$Q\left(\theta_{2}, k_{L}\right)$} & $Q\left(\theta_{2}, k_{H}\right)$ \\
\cline { 3 - 4 } & $Q\left(\theta_{1}, k_{L}\right)$ & $\theta_{1}, \theta_{2}$ & $\theta_{1}-k_{H}(1-\lambda \delta), \underline{\theta_{2}+k_{H}(1-\lambda \delta)}$ \\
\cline { 3 - 4 } & $Q\left(\theta_{1}, k_{H}\right)$ & $\underline{\theta_{1}}, \underline{\theta_{2}}$ \\
\cline { 3 - 4 } & &
\end{tabular}

Figure 17: Equilibrium when $\delta<\frac{1}{\lambda}$.

Therefore, I have shown that, under these simplifying assumptions, the condition required for determining the pure strategy Bayes-Nash equilibrium is the size of the probability of the neutral 
state relative to the size of the discount applied to negative deviations from target. Moreover, by Wilson (1971) since each case displays an odd number of equilibria, I can conclude that there are no non-degenerate mixed strategy equilibria. This completes the proof of Proposition 9 and Example 1.

\section{References}

Arad, A. and Rubinstein, A. Strategic Tournaments. American Economic Journal: Microeconomics, 5:31-54, 2013.

Borland, J. and MacDonald, R. Demand for Sport. Oxford Review of Economic Policy, 19: 478-502, 2003.

Che, Y.-K. and Gale, I. Rent Dissipation when Rent Seekers are Budget Constrained. Public Choice, 92:109-126, 1997.

Chiappori, P.-A., Levitt, S., and Groseclose, T. Testing Mixed-Strategy Equilibria When Players are Heterogeneous: The Case of Penalty Kicks in Soccer. American Economic Review, 92: 1138-1151, 2002.

Coloma, G. The Penalty Kick Game under Incomplete Information. Journal of Game Theory, 4:15-24, 2012.

Cox Jr., J. E. Approaches for Improving Salespersons' Forecasts. Industrial Marketing Management, 18:307-311, 1989.

Dixit, A. Strategic Behavior in Contests. The American Economic Review, 77:891-898, 1987.

El-Hodiri, M. and Quirk, J. An Economic Model of a Professional Sports League. Journal of Political Economy, 79:1302-1319, 1971.

Forrest, D. and Simmons, R. Outcome Uncertainty and Attendance Demand in Sport: The Case of English Soccer. Journal of the Royal Statistical Society. Series D(The Statistician), 51:229-241, 2002.

Fu, Q., Gürtler, O., and Münster, J. Communication and Commitment in Contests. Journal of Economic Behavior \& Orginization, 95:1-19, 2013.

Fudenberg, D. and Tirole, J. Game Theory. MIT Press, 1991.

Green, J. R. and Stokey, N. L. Rank-Order Tournaments and Contracts. Journal of Political Economy, 91:349-364, 1983.

Groh, C., Moldovanu, B., Sela, A., and Sunde, U. Optimal Seeding in Elinination Tournaments. Economic Theory, 49:59-80, 2012.

Harsanyi, J. C. Games with Incomplete Information Played by "Bayesian" players. Part I. The Basic Model. Management Science, 14:159-182, 1967. 
Harsanyi, J. C. Games with Incomplete Information Played by "Bayesian" players. Part II. Bayesian Equilibrium Points. Management Science, 14:320-334, 1968a.

Harsanyi, J. C. Games with Incomplete Information Played by "Bayesian" players. Part III. The Basic Probability Distribution of the Game. Management Science, 14:486-502, 1968b.

Herbig, P. A., Milewicz, J., and Golden, J. E. The Do's and Don'ts of Sales Forecasting. Industrial Marketing Management, 2:49-57, 1993.

Hossain, T. and Okui, R. The Binarized Scoring Rule. The Review of Economic Studies, 80: 984-1001, 2013.

Karni, E. A Mechanism for Eliciting Probabilities. Econometrica, 77:603-606, 2009.

Knowles, G., Sherony, K., and Haupert, M. The Demand for Major League Baseball: A Test of the Uncertainty of Outcome Hypothesis. The American Economist, 36:72-80, 1992.

Lazear, E. P. and Rosen, S. Rank-Order Tournaments as Optimum Labour Contracts. Journal of Political Economy, 89:841-864, 1981.

Mentzer, J. T. and Cox Jr., J. E. Familarity, Application, and Performance of Sales Forecasting Techniques. Journal of Forecasting, 3:27-36, 1984.

Nalebuff, B. J. and Stiglitz, J. E. Prizes and Incentives: Towards a General Theory of Compensation and Competition. The Bell Journal of Economics, 14:21-43, 1983.

Nash, J. Non-Cooperative Games. Annals of Mathematics, 54:286-295, 1951.

Neale, W. C. The Peculiar Economics of Professional Sports: A Contribution to the Theory of the Firm in Sporting Competition and in Market Competition. The Quarterly Journal of Economics, 78:1-14, 1964.

Nelson, R. G. and Bessler, D. A. Subjective Probabilities and Scoring Rules: Experimental Evidence. American Journal of Agricultural Economics, 71:363-369, 1989.

Nestor, J. Deep: Freediving, Renegade Science, and what the Ocean Tells us About Ourselves. Eamon Dolan/Houghton Mifflin Harcourt, 2014.

Pangallo, M., Sanders, J., Galla, T., and Farmer, J. D. A Taxonomy of Learning Dynamics in 2x2 Games. 2017.

Rottenberg, S. The Baseball Players' Labor Market. Journal of Political Economy, 64:242-258, 1956.

Savage, L. J. Elicitation of Personal Probabilities and Expectations. Journal of the American Statistical Association, 66:783-801, 1971.

Szymanski, S. The Economic Design of Sporting Contests. Journal of Economic Literature, 41: 1137-1187, 2003a. 
Szymanski, S. The Assessment: The Economics of Sport. Oxford Review of Economic Policy, 19:467-477, 2003b.

Vickrey, W. Counterspeculation, Auctions and Competitive Sealed Tenders. Journal of Finance, 16:8-39, 1961.

Vong, A. I. Strategic Manipulation in Tournament Games. Games and Economic Behavior, 1: $27-36,2017$.

Walker, M. and Wooders, J. Minimax Play at Wimbledon. American Economic Review, 91: 1521-1538, 2001.

Wilson, R. Computing Equilibria of N-Person Games. SIAM Journal on Applied Mathematics, 21:80-87, 1971. 